\title{
ON PRICING AND BUNDLING DECISIONS FOR STACKELBERG GAMES IN PARALLEL CHANNELS OF SUBSTITUTABLE COMPOSITES
}

\author{
Sina Keyhanian, AbBas Ahmadi* and Behrooz Karimi
}

\begin{abstract}
The paper describes competition within a supply network with parallel distribution channels. Each supply chain in the network is composed of a manufacturer and a retailer. Manufacturers sell two complementary products to the retailers, who then deliver to the end consumers. All players can bundle or not bundle their products assuming that the retail market presents the products in a mixed bundling setting. The motivation of this study is to mainly analyze the impact of cost reduction via manufacturers, on how the whole supply network will behave. We have modeled and solved partly and fully sequential game structures well known as Bertrand and Stackelberg games, where the preceding movers are considered to have more market power. Mathematical and numerical analyses reveal interesting propositions and managerial insights for decision makers who are practicing cost cutting strategies. The combination of different ordinal structures have led to exact mathematical comparisons among 24 games. Results indicate both manufacturers and retailers are better off with simultaneous pricing games. This promotes the concept of coordination through layer and channels of the network. Cost reduction with compensation increases payoffs when applied by the manufacturer whose complementary products' manufacturing costs are more distanced. It is also shown that retailers enjoy a retail advantage on one product at its best when playing retailer leading Stackelberg games.
\end{abstract}

Mathematics Subject Classification. 91A80, 91A40.

Received July 19, 2019. Accepted May 17, 2020.

\section{INTRODUCTION}

Managerial decisions are now more sophisticated than ever. Supply networks offer different opportunities for consumers to meet their needs. With the rise of startup companies, big companies have lost their monopolistic leadership in the market. The competitions are observed in different layers and distribution channels. The channels provide products with vertically differentiated features that also improve continuously through time. Many manufacturers' products such as Toyota and BMW are distinguishable among customers by the means of their vertical differentiation [16]. When a customer compares the two products, the various features of each automobile and their quality helps him/her make the purchasing decision. The aspect can also be widely observed in many service industries such as Uber [19] (ride-hailing companies in general) and airline services [14]. In fact, online services have grown so well, that it is now a common market behavior when competing new startup companies are launched on many areas, parallel to other incumbent companies.

Keywords. Supply chain management, pricing, bundling strategy, substitutable composites, Stackelberg games.

Department of Industrial Engineering and Management Systems, Amirkabir University of Technology, Tehran, Iran.

* Corresponding author: abbas.ahmadi@aut.ac.ir 
In such settings, substitutability and complementarity are the two important criteria that shape the market based on consumers' tastes. The consumers are likely to show interest if the complementary products are presented as bundles, and they will probably show more interest if they are free to choose from where they can buy the components of that bundle. The prices that retailers set on these individual or bundled products play a crucial role on how consumers' purchasing behavior can be. The retail prices themselves are affected by wholesale prices set by suppliers or manufacturers from an upstream level. Plurality of players leads to more complexity for decision makers. Nevertheless, especially under a decentralized setting where players seek to maximize their own benefit, it gives them the advantage to be able to examine various scenarios such as simultaneous or sequential moves, first or second mover's advantage and how these can be coordinated in order to maximize chain profits.

In this paper, we consider a supply network with two parallel channels, each including a manufacturer and a retailer. Each manufacturer produces two complementary products and only sells to one of the retailers. The products are requisite complementary to each other [22], therefore consumers are committed to purchase one unit of both. A realistic and tangible example of such products that are also inclined to form a composite is hardware and software, or basically mixed bundling of complementary information goods [23]. Mixed bundling [35], is a case where, customers can buy the components of the bundle separately, as well as a bundle. However, there is also an option of pure bundling [11], where the components of the bundle are not offered separately.

In service industries mixed bundling also appears to be an appealing option. For instance, in airlines and tourism [27], the service providers (considered as the suppliers or manufacturers), and the online agencies who have direct contact with customers and sell the offered packages (considered as retailers) fit the assumptions of this study.

We consider the corresponding products are also mutually substitutable making four available composites: two are direct complementary products and the other two are indirectly complementary to each other. The parallel structure of the supply network both in its inter-layer relations and product portfolio configuration necessitates the consideration of asymmetric settings for costs. By solving the supply network configuration under partially and fully sequential game structures, we have provided valuable managerial insights. Interestingly, some of our insights are aligned with previous studies, and some of them are not due to the existence of bundling strategy choice alongside with other assumptions. Some of the main ideas of this paper are inspired by a study done by Economides [8]. He proposed a same configuration of products in a duopoly under the concept of parallel vertical integration. By mathematical analysis he revealed that mixed bundling is the dominant strategy for both firms in a duopoly. In our paper, we try to extend these results for duopolistic manufacturers and retailers under parallel settings.

The main contributions of our paper are listed as follows:

(1) We approach the model of a supply network consisting in two competing parallel channels with sophisticated game models.

(2) We introduce the concept of cost reduction with compensation. This helps analyze the impact of cost reduction even in cost asymmetry settings.

(3) Three bundling structures are established for the supply chain, which is a combination of no bundling, pure or mixed bundling strategies for both manufacturers and retailers, eventually leading to 24 different game models, each optimized with exact solutions.

(4) We have considered market power (leader/follower) inside each game, and mathematically derived valuable propositions that shed light on how manufacturers and retailers behave on pricing and bundling decisions within different ordinal movements.

(5) We have specifically scrutinised the profit changes (gains) among different game structures/scenarios and revealed the tendencies of each player in the supply network.

The remainder of the paper is organized as follows: Section 2 provides the previous relevant research on the subject. Section 3 describes the problem. Section 4 discusses the Bertrand models of our paper, their game structures and optimal solutions. Section 5 presents a similar discussion for Stackelberg games with partially 
TABLE 1. Some relevant studies that consider decentralized models of pricing and/or bundling in duopolies or supply chains.

\begin{tabular}{lllllllllll}
\hline \hline Research & SM & R & M & NP & SIP & SCP & NB & PB & MB & CM \\
\hline Choi [7] & $\checkmark$ & 1 & 2 & 2 & $\checkmark$ & $\times$ & $\checkmark$ & $\times$ & $\times$ & $\times$ \\
Economides [8] & $\times$ & - & 2 & 4 & $\checkmark$ & $\checkmark$ & $\checkmark$ & $\checkmark$ & $\checkmark$ & $\times$ \\
Wu et al. [30] & $\checkmark$ & 2 & 1 & 2 & $\checkmark$ & $\times$ & $\checkmark$ & $\times$ & $\times$ & $\times$ \\
Wei et al. [28] & $\checkmark$ & 1 & 2 & 2 & $\checkmark$ & $\checkmark$ & $\checkmark$ & $\times$ & $\times$ & $\times$ \\
Chakravarty et al. [5] & $\times$ & 1 & $n$ & $n$ & $\checkmark$ & $\times$ & $\checkmark$ & $\checkmark$ & $\times$ & $\checkmark$ \\
Zhao et al. [34] & $\checkmark$ & 1 & 2 & 2 & $\checkmark$ & $\times$ & $\checkmark$ & $\times$ & $\times$ & $\checkmark$ \\
Giri et al. [10] & $\checkmark$ & 1 & 2 & 2 & $\checkmark$ & $\checkmark$ & $\checkmark$ & $\checkmark$ & $\times$ & $\times$ \\
Jafari et al. [13] & $\times$ & $n$ & 1 & 1 & $\checkmark$ & $\times$ & $\checkmark$ & $\times$ & $\times$ & $\checkmark$ \\
Pan and Zhou [24] & $\times$ & 1 & 1 & 2 & $\checkmark$ & $\checkmark$ & $\checkmark$ & $\checkmark$ & $\times$ & $\times$ \\
This article & $\checkmark$ & 2 & 2 & 4 & $\checkmark$ & $\checkmark$ & $\checkmark$ & $\checkmark$ & $\checkmark$ & $\times$ \\
\hline
\end{tabular}

Notes. SM: Sequence of moves, R: Retailer(s), M: Manufacturer(s), NP: Number of products, SIP: Sell individual products, SCP: Sell composite products, NB: No bundling, PB: Pure bundling, MB: Mixed bundling, CM: Centralized model(s) (Cooperative).

and fully sequential moves. Section 6 provides comparative analysis both mathematically and numerically on different possible aspects of the models alongside with mathematical propositions, corollaries and numerical insights. Section 7 summarizes results and findings, with recommendations for future possible developments and generalizations of this study.

\section{Literature REVIEW}

There are a number of studies relevant to pricing and bundling decisions, separately and together, in different game structures designed for supply chains with market power. Table 1 is a brief chronological list for comparison between some valuable studies in this field. In this paper, we use pure bundling and no bundling as the wholesale strategy at retailer-manufacturer interlayer, as well as mixed bundling and no bundling as the retail strategy at retailer-consumer level. A few set of works $[5,6,10]$, consider bundling for both retailers and manufacturers.

Keyhanian et al. [15] incorporate the composite product concept that is considering a demand group for consumers who want to buy both complementary products. Yan et al. [32] use a similar concept of individual and composite purchasing in an asymmetric setting with two retailers and one supplier. Choi [7] discusses different Bertrand and Stackelberg game structures with market powers for linear and nonlinear demands. The literature followed by his pioneer work, mostly consider manufacturer leading and retailer leading Bertrand (MSB and RSB), manufacturer leading and retailer leading Stackelberg (MSS and RSS) games. Some recent researchers approach the problem in the field of green supply chain. Peng and Zhang [21], assuming that one of the manufacturers practices green manufacturing, show that Bertrand models have the lowest outcomes for manufacturers while the retailers gain more profit. Xue and Zhang [31] discuss the benefits that retailers can achieve from an integrated supply chain of green products. They also conclude an equilibrium case of a power trade-off among the manufacturer and the retailer.

Wei et al. [28] perform a valuable study in regards to selling products separately and together. For functionally complementary products, they show that in both Bertrand and Stackelberg games the leaders achieve more profits, however, chain profit in both manufacturer and retailer leading Stackelberg games is higher than Bertrand games. Later, in a supply chain with duopolistic manufacturers, Zhao et al. [34] show that in Bertrand games, maximum profit is not affected by different market powers. Amir and Stepanova [3] discuss asymmetric linear costs in pricing decisions of a Bertrand duopoly where sequential moves are possible. They specifically work on the second mover's advantage, but also discuss in what conditions, whether the first or second mover's advantage survive. Aust and Buscher [4], find out that channel leadership is not always in the benefit of the 
manufacturer. They reach this insight in a duopoly of retailers with a common manufacturer where cooperation is also allowed.

Although assumed to be non-cooperative, decentralization can also come along with coordination and incentive alignment $[12,13,17]$. Many decentralized settings of supply chain discussed in literature consider centralizedbrought results as a benchmark for evaluating the price variables in different configurations. There are examples for both channel power structure pricing models in decentralized supply chains without bundling strategies $[18,20,26,29,33]$, and for pricing models with bundling strategies that have considered different game theoretic conditions along with centralized settings [10]. Some researches consider horizontal and vertical competition within the Bertrand and Stackelberg structures [30]. They determine ordinal relationships of optimal variables and also changes in profits as a function of retail substitutability.

\section{Problem Description}

In a dual channel and two-layer supply chain of two retailers (retailer 1 and retailer 2) and two manufacturers (manufacturer 1 and manufacturer 2), the manufacturers provide two base products, each at its own wholesale price. Manufacturer $i$ produces products $A i$ and $B i$ with unit manufacturing $\operatorname{costs} c_{A i}$ and $c_{B i}$, respectively and wholesales them to retailer $i$ at wholesale prices $w_{A i}, w_{B i}, i=1,2$. We consider that each retailer can only buy the products from one of the suppliers. Here, the supplier happens to be using the same index as the retailer. Retail prices, $p_{A i}$ and $p_{B i}$ are set by the retailers for the products they receive. Furthermore, assume that product types $A$ and $B$ are complementary products and that the corresponding products of suppliers are substitutable with each other in terms of their types, i.e. $A 1$ and $A 2$ are substitutable, $A 1$ and $B 1$ are complementary, $A 1$ and $B 2$ are complementary, $B 1$ and $B 2$ are substitutable. This setting is also known as parallel vertical integration [9].

The consumers purchase their desired products under a mixed-pure bundling (MPB) setting. That is, they are bound to buy a composite product consisting of both $A$ and $B$; however from which retailer to buy each of these two base product types from, is an arbitrary choice for the consumers. A consumer may buy both product types $A$ and $B$ from retailer $i$, or buy product type $A$ from retailer $i$ and product type $B$ from the other retailer. Product $A$ is useless without its complementary product $B$ and vice versa.

The concept of mixed-pure bundling is fairly different from the mixed bundling used in the literature [8,25]. In the bundling literature, mixed bundling means the consumers can buy the bundle or the components separately. A consumer either buys a bundle or a composite of two complementary products. In fact, this is a mixed bundling strategy at retailer/manufacturer level which comes out to be a pure bundling offer in the consumer level; whether in the context of bundles or composites.

The firms that decide to bundle can only bundle their own products; own-bundling. Cross-bundling is not allowed due to decentralization. Based on bundling literature $[8,25]$ the available strategies are pure, mixed and no bundling which we have arranged them into two strategies of mixed-pure bundling and no bundling. If one bundles products $A i$ and $B j,(i, j) \in\{1,2\} \times\{1,2\}$, based on the fact that whether it is wholesaling or retailing, then the prices of the bundle would be $v_{i j}$ or $s_{i j}$, respectively. The bundle prices should apply in the inequalities $v_{i j}<w_{A i}+w_{B j}$ and $s_{i j}<p_{A i}+p_{B j}$; otherwise, the bundling assumption will not qualify as a distinct strategy, [1]. All the firms have prefect information about demands and costs and seek to maximize their own profits. This assumption leaves the problem with decentralized structures. The supply chain is depicted as given in Figure 1.

Let $s_{i j}^{n b}=p_{A i}+p_{B j}$ denote the price of a composite consisting of retailer $i$ 's product $A$ and retailer $j$ 's product $B$, which is not offered as a bundle and thus equals the sum of its components' prices. Let $s_{i j}^{b}<p_{A i}+p_{B j}$ denote the price of that same composite which is now offered as a bundle. The general demand function of a composite is given as follows:

$$
D_{i j}=\alpha-\beta s_{i j}+\gamma \sum_{\substack{(m, n) \in\{(1,1),(1,2),(2,1),(2,2)\} \\ \wedge(m, n) \neq(i, j)}} s_{m n},
$$




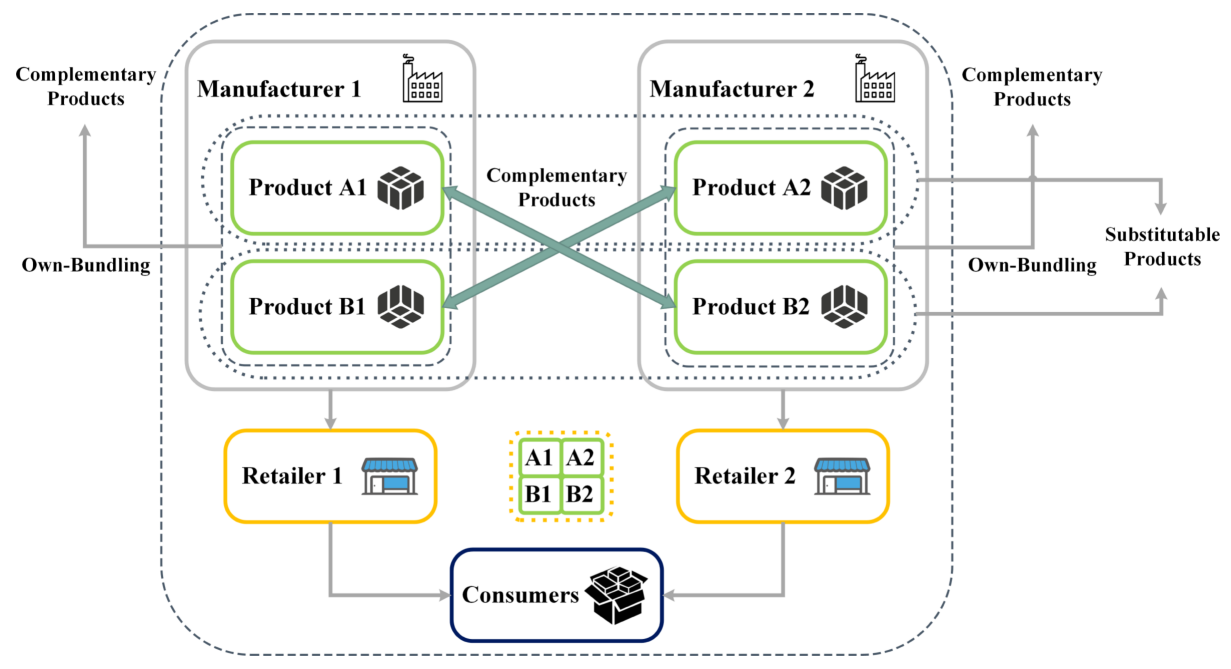

FiguRe 1. A dual channel supply chain with complementary and substitutable products.

where $D_{i j}$ is the demand of the composite in which product type $A$ is purchased from retailer $i$ and product type $B$ is purchased from retailer $j$, parameter $\alpha$ is the intercept, $\beta$ and $\gamma$ are the price-effect and cross-price effect respectively.

We use the term composites deliberately to avoid getting mistaken with bundling, as the consumer might buy the two products $A$ and $B$ from separate firms while they are not offered as a bundle. If offered as a bundle, still the couple would form a composite. In other words, the defined market can offer two types of bundles and four types of non-bundle composites. The bundles are $A 1 B 1$ and $A 2 B 2$ at retail prices $s_{11}$ and $s_{22}$, and wholesale prices $v_{11}$ and $v_{22}$, respectively. The non-bundle composites are $A 1 B 2$ and $A 2 B 1$ and in case not provided as bundles, $A 1 B 1$ and $A 2 B 2$ also each form a non-bundle composite. For example, if products $A 1$ and $B 1$, and $A 2$ and $B 2$ are both offered as bundles by the retailers, the set of demand functions would be as shown in equation (3.2).

$$
\begin{aligned}
& D_{11}=\alpha-\beta s_{11}+\gamma\left(p_{B 1}+p_{A 2}\right)+\gamma\left(p_{A 1}+p_{B 2}\right)+\gamma s_{22} \\
& D_{12}=\alpha-\beta\left(p_{A 1}+p_{B 2}\right)+\gamma\left(p_{B 1}+p_{A 2}\right)+\gamma s_{11}+\gamma s_{22} \\
& D_{21}=\alpha-\beta\left(p_{B 1}+p_{A 2}\right)+\gamma\left(p_{A 1}+p_{B 2}\right)+\gamma s_{11}+\gamma s_{22} \\
& D_{22}=\alpha-\beta s_{22}+\gamma\left(p_{B 1}+p_{A 2}\right)+\gamma\left(p_{A 1}+p_{B 2}\right)+\gamma s_{11} .
\end{aligned}
$$

This formulation was used by Economides [8], to discuss bundle pricing in the case of retailer competition. Similar to that study, we also assume that increasing prices of all four composites will decrease their demands which leads us to the assumption of $\beta \geq 3 \gamma$.

In case of calculating $D_{11}$ for instance, the demand has a negative relation with bundle $\{A 1, B 1\}$ 's price which is $s_{11}<p_{A 1}+p_{B 1}$, and positive relation with the other competing composites consisting of nonbundles $\{A 1, B 2\},\{A 2, B 1\}$ priced at $p_{A 1}+p_{B 2}$ and $p_{A 2}+p_{B 1}$, respectively, and bundle $\{A 2, B 2\}$ priced at $s_{22}<p_{A 2}+p_{B 2}$. The wholesale prices are not present in demand settings. They show their impact when we evaluate both manufacturers and retailers profit. This is dependent on whether the manufacturers bundle the offered composites or not. For example, if the manufacturers also bundle the composites (knowing that the retailers also have bundled, as in case MBRB in Fig. 4), then the profits gained by each manufacturer and retailer are calculated as shown in equation (3.3).

$$
\begin{aligned}
& \pi_{M 1}=\left(v_{11}-c_{A 1}-c_{B 1}\right) D_{11}+\left(w_{A 1}-c_{A 1}\right) D_{12}+\left(w_{B 1}-c_{B 1}\right) D_{21} \\
& \pi_{M 2}=\left(w_{B 2}-c_{B 2}\right) D_{12}+\left(w_{A 2}-c_{A 2}\right) D_{21}+\left(v_{22}-c_{A 2}-c_{B 2}\right) D_{22} \\
& \pi_{R 1}=D_{11}\left(s_{11}-v_{11}\right)+D_{12}\left(p_{A 1}-w_{A 1}\right)+D_{21}\left(p_{B 1}-w_{B 1}\right) \\
& \pi_{R 2}=D_{22}\left(s_{22}-v_{22}\right)+D_{21}\left(p_{A 2}-w_{A 2}\right)+D_{12}\left(p_{B 2}-w_{B 2}\right)
\end{aligned}
$$


TABle 2. Structures of games and their corresponding decision variables, due to different bundling decisions among each layer of the supply network.

\begin{tabular}{|c|c|c|c|c|c|}
\hline MBD & RBD & Abbreviation & Figure & MDV & RDV \\
\hline $\mathrm{N}$ & $\mathrm{N}$ & MNRN & 4 , first scenario from left & $\begin{array}{l}w_{A 1}, w_{B 1}, \\
w_{A 2}, w_{B 2} .\end{array}$ & $\begin{array}{l}p_{A 1}, p_{B 1}, \\
p_{A 2}, p_{B 2}\end{array}$ \\
\hline $\mathrm{N}$ & MB & MNRB & 4 , second scenario from left & $\begin{array}{l}w_{A 1}, w_{B 1} \\
w_{A 2}, w_{B 2}\end{array}$ & $\begin{array}{l}p_{A 1}, p_{B 1}, \\
p_{A 2}, p_{B 2}, \\
s_{11}, s_{22} .\end{array}$ \\
\hline MB & MB & MBRB & 4 , third scenario from left & $\begin{array}{l}w_{A 1}, w_{B 1} \\
w_{A 2}, w_{B 2} \\
v_{11}, v_{22}\end{array}$ & $\begin{array}{l}p_{A 1}, p_{B 1}, \\
p_{A 2}, p_{B 2}, \\
s_{11}, s_{22} .\end{array}$ \\
\hline
\end{tabular}

Notes. RBD: Retailers' bundling decision (N: not bundling, MB: mixed bundling), MBD: Manufacturers' bundling decision (N: not bundling, B: mixed bundling), RDV: Retailers' decision variables, MDV: Manufacturers' decision variables.

where for $i \in\{1,2\}, \pi_{M i}$ and $\pi_{R i}$ represent profits of manufacturer $i$ and retailer $i, w_{A i}$ and $w_{B i}$ represent wholesale prices set by manufacturer $i$ for products $A$ and $B$, and, $c_{A i}$ and $c_{B i}$ represent production costs incurred by manufacturer $i$ to manufacture products $A$ and $B$, respectively.

For instance, for manufacturer 1 (M1), the sales is consisted of selling bundle $\{A 1, B 1\}$, and selling them separately. Manufacturer 1's separate sales of product $A$ corresponds to $D_{12}$ (the consumers who buy product $A$ from retailer 1 and product $B$ from retailer 2), and his/her separate sales of product $B$ corresponds to $D_{21}$ (the consumers who buy product $A$ from retailer 2 and product $B$ from retailer 1). However, manufacturer 1's sales of the bundle $\{A 1, B 1\}$ corresponds to $D_{11}$ where she also incurs both $\operatorname{costs} c_{A i}, c_{B i}$. The cost that retailers incur, is actually the wholesale price at which they bought the products from their corresponding manufacturer.

Here we highlight the differences among the game structures designed in this study. Each game's structure is a combination of market power, move sequences and bundling decisions. The different bundling decisions that happen in each layers of supply network are shown in Table 2. Because the customers are also offered with the choice to buy the products separately from whichever retailer they prefer, in all cases where bundling happens, it is in fact in a form of mixed bundling. Therefore both individual product prices and their bundle prices are present in the modeling in those cases.

Market power and move sequences might seem to be describing the same concept, but in this study we use a different point of view for each. They trigger two different types of Stackelberg behavior in a supply network. Market power undertakes the inter-layer Stackelberg games in which leadership is in control of retailers or manufacturers. While, move sequences represent the intra-layer Stackelberg games in which for example, within the manufacturing level, manufacturer 1 sets his/her prices before manufacturer 2. Meanwhile Bertrand games reflect the concept when for a layer, these move sequences are merged into simultaneous moves. In order to comprehensively capture the behavior of move sequences in the supply network, we consider all possible and distinct permutations of market power, sequence moves and bundling decisions through inter-layer and intralayer relations in the supply network.

The Bertrand games describe the following situations (Fig. 3).

- MSB: In this structure, manufacturers set prices simultaneously, and retailers set prices simultaneously. However, the manufacturers hold market leadership/more market power. Therefore, first the manufacturers set their prices and then it is the retailers' turn to set their prices.

- RSB: In this structure, retailers set prices simultaneously and manufacturers set prices simultaneously. However, the retailers hold market leadership/more market power. Therefore, first the retailers set their prices and then it is the manufacturers' turn to set their prices. 
In MSS-RS and RSS-MS games both Bertrand games and Stackelberg games are present in most parts of the game. First a Stackelberg game is played within the "leader" layer, then a Bertrand game is played within the "follower" layer.

- MSS-RS: In this structure, manufacturers set prices sequentially, while retailers set prices simultaneously. Meanwhile, the manufacturers also hold market leadership/more market power than the retailers. However, without loss of generality (w.l.o.g) it is assumed that within the manufacturing layer, manufacturer 1 sets his/her price first and then manufacturer 2 sets his/her price. After that, the retailers set their prices.

- RSS-MS: In this structure, retailers set prices sequentially, while manufacturers set prices simultaneously. Meanwhile, the retailers also hold market leadership/more market power than the manufacturers. However, w.l.o.g it is assumed that within the retailing layer, retailer 1 sets his/her price first and then retailer 2 sets his/her price. After that, the manufacturers set their prices.

In MSS and RSS games no simultaneous moves are present and all of the game moves are completely sequential, whether within layers or between the two layers of supply network. Distinct permutations of these sequential moves have led us to add a Greek numbering suffix to each of the structures in order to distinguish their differences.

- MSSi\&ii: This structure represents MSSi and MSSii which however are two different cases, but w.l.o.g reflect the same insights. In both of these structures the manufacturers hold market leadership/more market power than the retailers. For example, in MSSi first manufacturer 1 sets his/her price, then manufacturer 2, then the retailer 2 who is in the same supply chain that manufacturer 2 appears to be sets his/her price, and finally retailer 1 sets his/her price. The same trail happens in MSSii but in reverse starting from manufacturer 2 setting his/her price.

- MSSiii\&iv: This structure represents MSSiii and MSSiv which however are two different cases, but w.l.o.g reflect the same insights. Again, in both of these structures the manufacturers hold market leadership/more market power than the retailers. For example, in MSSiii first manufacturer 1 sets his/her price, then manufacturer 2. But this time, retailer 1 who is in the opposite supply chain, makes the next move, and finally retailer 2 sets his/her price. The same trail happens in MSSiv but in reverse starting from manufacturer 2 setting his/her price.

- RSSi\&ii: This structure represents RSSi and RSSii which however are two different cases, but w.l.o.g reflect the same insights. In both of these structures the retailers hold market leadership/more market power than the manufacturers. For example, in RSSi first retailer 1 sets his/her price, then retailer 2, then the manufacturer 2 who is in the same supply chain that retailer 2 appears to be sets his/her price, and finally manufacturer 1 sets his/her price. The same trail happens in RSSii but in reverse starting from retailer 2 setting his/her price.

- RSSiii\&iv: This structure represents RSSiii and RSSiv which however are two different cases, but w.l.o.g reflect the same insights. Again, in both of these structures the retailers hold market leadership/more market power than the manufacturers. For example, in RSSiii first retailer 1 sets his/her price, then retailer 2. But this time, manufacturer 1 who is in the opposite supply chain, makes the next move, and finally manufacturer 2 sets his/her price. The same trail happens in RSSiv but in reverse starting from retailer 2 setting his/her price.

These structures form 24 different game structures that we use to perform the comparative analysis among games and extract behavioral insights of different players within the supply network.

\section{Decisions in Bertrand games}

\subsection{The manufacturing leading Bertrand game (MSB)}

When the manufacturers are more powerful, they set the prices first and based on those prices the retailers set their optimal prices. Therefore, in order to maximize the manufacturers' profit, we have to assume a backward 


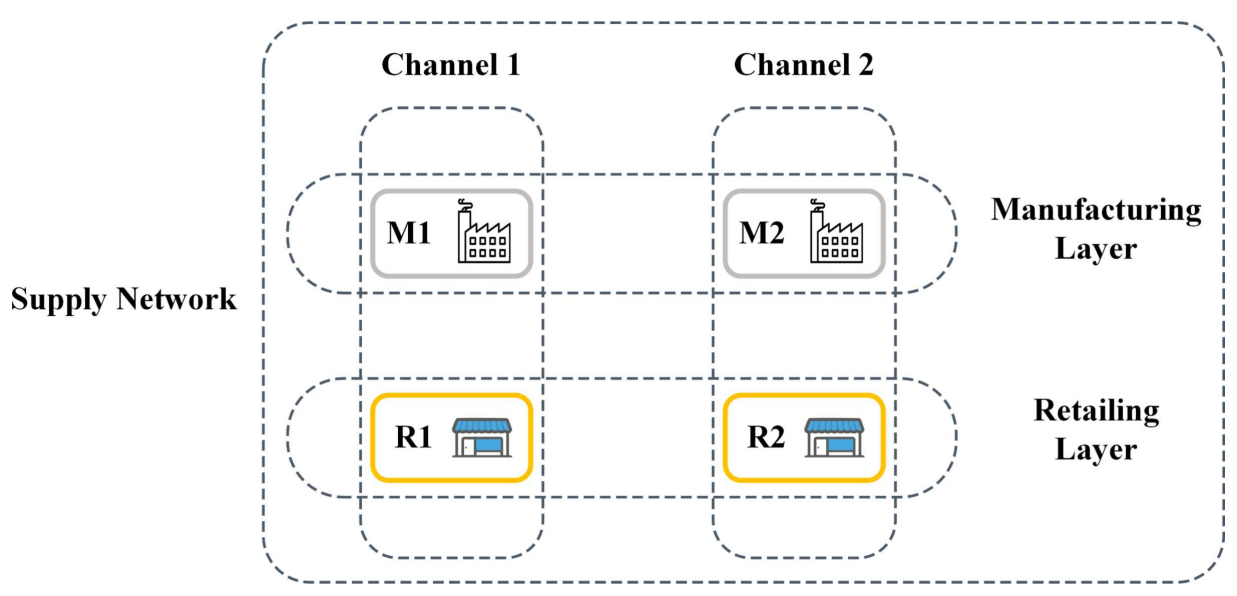

FiguRE 2. The structure of channels and layers in the whole supply network.

method. First the best response retail prices to any sets of wholesale prices are obtained. Then based on those prices, the optimal wholesale prices and profits of manufacturers are calculated. The structure of the game is shown in equation (4.1).

$$
\begin{aligned}
& \left\{\begin{array}{c}
\max _{w_{A 1}, w_{B 1}} \pi_{M 1}\left(w_{A 1}, w_{B 1}, p_{A 1}^{*}(\vec{w}), p_{B 1}^{*}(\vec{w}), p_{A 2}^{*}(\vec{w}), p_{B 2}^{*}(\vec{w})\right) \\
\max _{w_{A 2}, w_{B 2}} \pi_{M 2}\left(w_{A 2}, w_{B 2}, p_{A 1}^{*}(\vec{w}), p_{B 1}^{*}(\vec{w}), p_{A 2}^{*}(\vec{w}), p_{B 2}^{*}(\vec{w})\right)
\end{array},\right. \\
& \Leftarrow\left\{\begin{array}{l}
\left(p_{A 1}^{*}(\vec{w}), p_{B 1}^{*}(\vec{w})\right)=\underset{\left(p_{A 1}, p_{B 1}\right)}{\arg \max } \pi_{R 1}\left(p_{A 1}, p_{B 1}, w_{A 1}, w_{B 1}\right), \\
\left(p_{A 2}^{*}(\vec{w}), p_{B 2}^{*}(\vec{w})\right)=\underset{\left(p_{A 2}, p_{B 2}\right)}{\arg \max } \pi_{R 2}\left(p_{A 2}, p_{B 2}, w_{A 2}, w_{B 2}\right) .
\end{array}\right.
\end{aligned}
$$

The vector $\vec{w}$ represents quadruple $\left(w_{A 1}, w_{B 1}, w_{A 2}, w_{B 2}\right)$. The left arrow in equation (4.1) demonstrates a reverse approach for response optimization. Because manufacturers are the price leaders (first movers) here, they have the benefit of optimizing their response (wholesale prices) based on the estimated response of retailers (retail prices). Therefore, equation (4.1) first captures the optimal retail prices assuming that wholesale prices are set by the manufacturers and thus known to the retailers. The outcome will be off course in terms of the known variables of the system which are the components of $\vec{w}$. However, if the retailers or manufacturers choose to bundle their products, the variables $s_{11}, s_{22}$ and $v_{11}, v_{22}$ will be added to the formation in equation (4.1). After achieving the optimal equations of retail prices, by replacing these equations in their relevant variable in set of profit functions like equation (3.3), we have a set of equations that are purely in terms of wholesale prices (whether separate wholesale prices labeled by $w$, or bundle wholesale prices labeled by $v$, or both). In Section 4.2, it is shown that how this result is achieved (Figs. 2 and 3).

\subsection{No bundling in the MSB game}

When neither manufacturers nor retailers offer bundling (case MNRN in Fig. 4), the demand and profit functions are given by equations (4.2) and (4.3), respectively. Because of the absence of bundling, variables $s$ and $v$, which reflect the bundle prices for retailers and manufacturers, respectively, are not present in these 

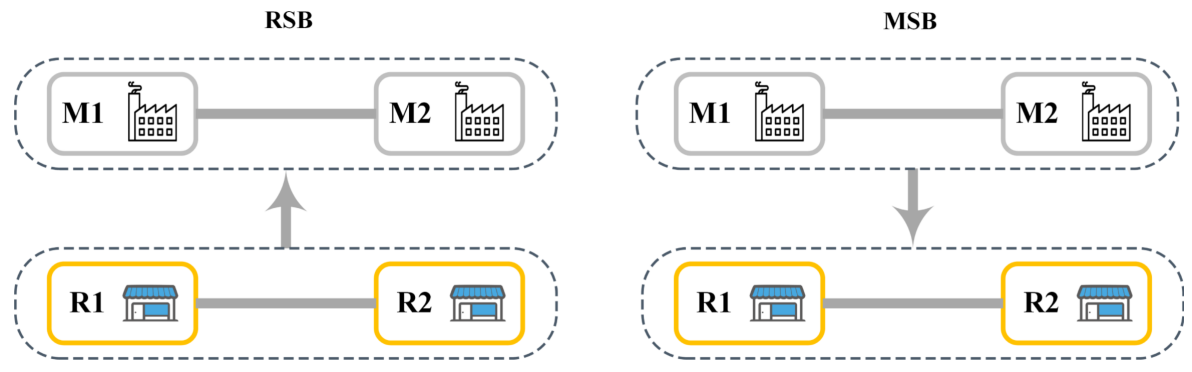

Figure 3. The RSB game (left) and MSB game (right) structures.

equations.

$$
\begin{aligned}
D_{11} & =\alpha-\beta \times\left(p_{A 1}+p_{B 1}\right)+\gamma \times\left(p_{A 1}+p_{B 2}\right)+\gamma \times\left(p_{A 2}+p_{B 1}\right)+\gamma \times\left(p_{A 2}+p_{B 2}\right) \\
D_{12} & =\alpha-\beta \times\left(p_{A 1}+p_{B 2}\right)+\gamma \times\left(p_{A 1}+p_{B 1}\right)+\gamma \times\left(p_{A 2}+p_{B 2}\right)+\gamma \times\left(p_{A 2}+p_{B 1}\right) \\
D_{21} & =\alpha-\beta \times\left(p_{A 2}+p_{B 1}\right)+\gamma \times\left(p_{A 2}+p_{B 2}\right)+\gamma \times\left(p_{A 1}+p_{B 1}\right)+\gamma \times\left(p_{A 1}+p_{B 2}\right) \\
D_{22} & =\alpha-\beta \times\left(p_{A 2}+p_{B 2}\right)+\gamma \times\left(p_{A 2}+p_{B 1}\right)+\gamma \times\left(p_{A 1}+p_{B 2}\right)+\gamma \times\left(p_{A 1}+p_{B 1}\right) \\
\pi_{M 1} & =\left(w_{A 1}-c_{A 1}\right)\left(D_{11}+D_{12}\right)+\left(w_{B 1}-c_{B 1}\right)\left(D_{11}+D_{21}\right) \\
\pi_{M 2} & =\left(w_{A 2}-c_{A 2}\right)\left(D_{21}+D_{22}\right)+\left(w_{B 2}-c_{B 2}\right)\left(D_{12}+D_{22}\right) \\
\pi_{R 1} & =\left(p_{A 1}-w_{A 1}\right)\left(D_{11}+D_{12}\right)+\left(p_{B 1}-w_{B 1}\right)\left(D_{11}+D_{21}\right) \\
\pi_{R 2} & =\left(p_{A 2}-w_{A 2}\right)\left(D_{21}+D_{22}\right)+\left(p_{B 2}-w_{B 2}\right)\left(D_{12}+D_{22}\right) .
\end{aligned}
$$

By replacing the demands in equation (4.3), the profit functions are simplified in terms of prices as shown in equation (4.4).

$$
\begin{aligned}
\pi_{M 1}= & \left(w_{B 1}-c_{B 1}\right)\left(2 \alpha-(\beta-3 \gamma) p_{A 1}-(\beta-3 \gamma) p_{A 2}-(2 \beta-2 \gamma) p_{B 1}+4 \gamma p_{B 2}\right) \\
& +\left(w_{A 1}-c_{A 1}\right)\left(2 \alpha-(2 \beta-2 \gamma) p_{A 1}+4 \gamma p_{A 2}-(\beta-3 \gamma) p_{B 1}-(\beta-3 \gamma) p_{B 2}\right) \\
\pi_{M 2}= & \left(w_{B 2}-c_{B 2}\right)\left(2 \alpha-(\beta-3 \gamma) p_{A 2}-(\beta-3 \gamma) p_{A 1}-(2 \beta-2 \gamma) p_{B 2}+4 \gamma p_{B 1}\right) \\
& +\left(w_{A 2}-c_{A 2}\right)\left(2 \alpha-(2 \beta-2 \gamma) p_{A 2}+4 \gamma p_{A 1}-(\beta-3 \gamma) p_{B 2}-(\beta-3 \gamma) p_{B 1}\right) \\
\pi_{R 1}= & \left(p_{A 1}-w_{A 1}\right)\left(2 \alpha-2(\beta-\gamma) p_{A 1}+4 \gamma p_{A 2}-(\beta-3 \gamma) p_{B 1}-(\beta-3 \gamma) p_{B 2}\right) \\
& +\left(p_{B 1}-w_{B 1}\right)\left(2 \alpha-(\beta-3 \gamma) p_{A 1}-(\beta-3 \gamma) p_{A 2}-2(\beta+\gamma) p_{B 1}+4 \gamma p_{B 2}\right) \\
\pi_{R 2}= & \left(p_{A 2}-w_{A 2}\right)\left(2 \alpha-2(\beta-\gamma) p_{A 2}+4 \gamma p_{A 1}-(\beta-3 \gamma) p_{B 2}-(\beta-3 \gamma) p_{B 1}\right) \\
& +\left(p_{B 2}-w_{B 2}\right)\left(2 \alpha-(\beta-3 \gamma) p_{A 2}-(\beta-3 \gamma) p_{A 1}-2(\beta+\gamma) p_{B 2}+4 \gamma p_{B 1}\right) .
\end{aligned}
$$

Based on the response approach in equation (4.1), first we optimize the profits in terms of retail prices (all prices labeled with $p$ ) with the assumption that the wholesale prices (all prices labeled with $w$ ) are given by the manufacturers. Retailer 1 has control of prices $p_{A 1}, p_{B 1}$ and retailer 2 has control of prices $p_{A 2}, p_{B 2}$. In other words, each of retailer has two responses. Therefore, the potentially optimal retail prices or stationary points can be obtained by solving:

$$
\frac{\partial \pi_{R 1}\left(p_{A 1}, p_{B 1}\right)}{p_{A 1}}=\frac{\partial \pi_{R 1}\left(p_{A 1}, p_{B 1}\right)}{p_{B 1}}=\frac{\partial \pi_{R 2}\left(p_{A 2}, p_{B 2}\right)}{p_{A 2}}=\frac{\partial \pi_{R 2}\left(p_{A 2}, p_{B 2}\right)}{p_{B 2}}=0 .
$$

Proposition 4.1. The profit functions of retailers are concave. Therefore, the stationary points of equation (4.5) are indeed the retail prices that maximize their corresponding profit functions. 
The proof of Proposition 4.1 can be found in Appendix A.1. Because all values but retail prices are assumed to be given, simplifying equation (4.5), leads to four linear equations in terms of four different variables:

$$
\begin{aligned}
& \left(\begin{array}{cccc}
-4(\beta+\gamma) & -2(\beta-3 \gamma) & 4 \gamma & -\beta+3 \gamma \\
-2(\beta-3 \gamma) & -4(\beta+\gamma) & -\beta+3 \gamma & 4 \gamma \\
4 \gamma & -\beta+3 \gamma & -4(\beta+\gamma) & -2(\beta-3 \gamma) \\
-\beta+3 \gamma & 4 \gamma & -2(\beta-3 \gamma) & -4(\beta+\gamma)
\end{array}\right)\left(\begin{array}{l}
p_{A 1}^{*} \\
p_{B 1}^{*} \\
p_{A 2}^{*} \\
p_{B 2}^{*}
\end{array}\right) \\
& +\left(\begin{array}{l}
2 \alpha+2(\beta-\gamma) w_{A 1}+(\beta-3 \gamma) w_{B 1} \\
2 \alpha+(\beta-3 \gamma) w_{A 1}+2(\beta-\gamma) w_{B 1} \\
2 \alpha+2(\beta-\gamma) w_{A 1}+(\beta-3 \gamma) w_{B 1} \\
2 \alpha+(\beta-3 \gamma) w_{A 2}+2(\beta-\gamma) w_{B 2}
\end{array}\right)=0
\end{aligned}
$$

Using Gaussian elimination [2], we obtain $p^{*}$. For instance, $p_{A 1}^{*}$ is shown in equation (4.7). All wholesale prices are available in the optimal response. This means each retail price takes effect from both manufacturers' wholesale prices. Due to the symmetric relations among prices of substitutable and complementary products, the other solutions also have a same structure.

$$
\begin{aligned}
p_{A 1}^{*}= & -\frac{1}{3\left(35 \beta^{2}-106 \beta \gamma+51 \gamma^{2}\right)} \\
& \times\left(-30 \alpha \beta-62 w_{A 1} \beta^{2}-13 w_{A 2} \beta^{2}+8 w_{B 1} \beta^{2}\right. \\
& +22 w_{B 2} \beta^{2}+18 \alpha \gamma+196 w_{A 1} \beta \gamma+14 w_{A 2} \beta \gamma \\
& -16 w_{B 1} \beta \gamma-92 w_{B 2} \beta \gamma-126 w_{A 1} \gamma^{2}+27 w_{A 2} \gamma^{2} \\
& \left.-24 w_{B 1} \gamma^{2}+78 w_{B 2} \gamma^{2}\right) .
\end{aligned}
$$

We simplify and rearrange all solutions such that the denominators are factored and coefficients are separated price-wise. This helps significantly with the comparative analyses implemented in this research. For example, the factor of $35 \beta^{2}-106 \beta \gamma+51 \gamma^{2}$ is $(5 \beta-3 \gamma)(7 \beta-17 \gamma)$. Therefore, $\frac{-1}{3(5 \beta-3 \gamma)(7 \beta-17 \gamma)}$ is the common denominator for all optimal retail price responses. The expressions that are independent of the wholesale prices in the parenthesis multiplied by the common denominator, are $-30 \alpha \beta+18 \alpha \gamma=-6 \alpha(5 \beta-3 \gamma)$ which after multiplication, becomes $\frac{2 \alpha}{(7 \beta-17 \gamma)}$ revealing the constant of the optimal retail price response. Finally, $\forall k \in\{A, B\}, i \in\{1,2\}$, the best response retail prices in terms of predetermined wholesale prices are:

$$
\begin{aligned}
p_{k i}^{*}(\vec{w})= & \frac{2 \alpha}{(7 \beta-17 \gamma)}+\frac{62 \beta^{2}-196 \beta \gamma+126 \gamma^{2}}{3(5 \beta-3 \gamma)(7 \beta-17 \gamma)} w_{k i}+\frac{13 \beta^{2}-14 \beta \gamma-27 \gamma^{2}}{3(5 \beta-3 \gamma)(7 \beta-17 \gamma)} w_{k, 3-i} \\
& -\frac{8 \beta^{2}-24 \gamma^{2}-16 \beta \gamma}{3(5 \beta-3 \gamma)(7 \beta-17 \gamma)} w_{\{A, B\} \backslash k, i}-\frac{22 \beta^{2}-92 \beta \gamma+78 \gamma^{2}}{3(5 \beta-3 \gamma)(7 \beta-17 \gamma)} w_{\{A, B\} \backslash k, 3-i} .
\end{aligned}
$$

Because the manufacturers' profit functions are dependent on demands, they are also dependent on retail prices. By replacing the above best response retail prices in $\pi_{M 1}$ and $\pi_{M 2}$, we obtain the manufacturers' profit functions in terms of wholesale price variables ( $c f$. Appendix A). Therefore, the potentially optimal wholesale prices or stationary points can be obtained by solving:

$$
\frac{\partial \pi_{M 1}\left(w_{A 1}, w_{B 1}\right)}{w_{A 1}}=\frac{\partial \pi_{M 1}\left(w_{A 1}, w_{B 1}\right)}{w_{A 1}}=\frac{\partial \pi_{M 2}\left(w_{A 2}, w_{B 2}\right)}{w_{A 2}}=\frac{\partial \pi_{M 2}\left(w_{A 2}, w_{B 2}\right)}{w_{B 2}}=0 .
$$


A system of linear equations will be obtained similar to equation (4.6) for wholesale prices. The optimal wholesale prices $\forall k \in\{A, B\}, i \in\{1,2\}$ are:

$$
\begin{aligned}
w_{k i}^{*}= & \frac{406 c_{k i}+221 c_{k, 3-i}-408 c_{\{A, B\} \backslash k, i}-186 c_{\{A, B\} \backslash k, 3-i}}{1221} \\
& +\frac{148\left(c_{k i}-c_{k, 3-i}+c_{\{A, B\} \backslash k, i}-c_{\{A, B\} \backslash k, 3-i}\right) \beta(37 \beta-99 \gamma)}{1221\left(31 \beta^{2}-66 \beta \gamma-33 \gamma^{2}\right)} \\
& +\frac{66\left(2 \beta(37 \beta-99 \gamma) \sum_{\substack{n \in\{A, B\} \\
j \in\{1,2\}}} c_{n j}+37 \alpha(5 \beta-3 \gamma)\right)}{1221\left(37 \beta^{2}-118 \beta \gamma+37 \gamma^{2}\right)} .
\end{aligned}
$$

$\{A, B\} \backslash k$ represents the element which remains after subtracting $k$ from set $\{A, B\}$. For example, if $k=A$, then $\{A, B\} \backslash A=B$, and $c_{\{A, B\} \backslash k, i}=c_{B i}$.

Proposition 4.2. The profit functions of manufacturers are concave. Therefore, the stationary points of equation (4.10) are indeed the wholesale prices that maximize their corresponding profit functions.

The proof of Proposition 4.2 can be found in Appendix A.2. The optimal wholesale prices are not symmetric because of the difference between unit manufacturing costs. But regardless of that, a symmetry can be seen within the solution. The coefficients switch places wherever the corresponding manufacturing cost is. Also notice a linear combination of costs and multiples of the expressions $\sum_{n \in\{A, B\}} \sum_{j \in\{1,2\}} c_{n j}$ and $\left(c_{k i}-c_{k, 3-i}+c_{\{A, B\} \backslash k, i}-c_{\{A, B\} \backslash k, 3-i}\right)$. These three statements and their multiples are present in all the optimal solutions helping us to ease the mathematical analysis. The optimal product retail prices are derived by replacing equation (4.10) in equation (4.8). All the optimal solutions of this subsection that were not presented in exact form, have been provided explicitly in Appendix B.

\subsection{Bundling in the MSB game}

For the MSB game when manufacturers do not bundle and retailers bundle (case MNRB in Fig. 4), variables $s_{11}$ and $s_{22}$ are added to the profit function as in equation (3.1). The demand functions are as in equation (3.2), manufacturers' profits are as in equation (4.3), and retailers' profits are as in equation (3.3). With the same approach used in result of Proposition 4.1, and equation (4.6), the best response retail prices are calculated as follows:

$$
\begin{aligned}
p_{k i}^{*}(\vec{w})= & \frac{2 \alpha}{3(2 \beta-5 \gamma)}+\frac{8 \beta^{2}-34 \beta \gamma+36 \gamma^{2}}{3(2 \beta-5 \gamma)(2 \beta-3 \gamma)} w_{k i}+\frac{\gamma(4 \beta-9 \gamma)}{3(2 \beta-5 \gamma)(2 \beta-3 \gamma)} w_{k, 3-i} \\
& -\frac{2 \gamma(\beta-3 \gamma)}{3(2 \beta-5 \gamma)(2 \beta-3 \gamma)} w_{\{A, B\} \backslash k, i}-\frac{4 \beta^{2}-20 \beta \gamma+24 \gamma^{2}}{3(2 \beta-5 \gamma)(2 \beta-3 \gamma)} w_{\{A, B\} \backslash k, 3-i} . \\
s_{i i}^{b *}(\vec{w})= & \frac{\alpha}{(2 \beta-5 \gamma)}+\frac{2(\beta-2 \gamma)^{2}}{(2 \beta-5 \gamma)(2 \beta-3 \gamma)} w_{A i}+\frac{2(\beta-2 \gamma)^{2}}{(2 \beta-5 \gamma)(2 \beta-3 \gamma)} w_{B i} \\
& +\frac{\gamma(\beta-2 \gamma)}{(2 \beta-5 \gamma)(2 \beta-3 \gamma)} w_{A, 3-i}+\frac{\gamma(\beta-2 \gamma)}{(2 \beta-5 \gamma)(2 \beta-3 \gamma)} w_{B, 3-i} .
\end{aligned}
$$


MNRN

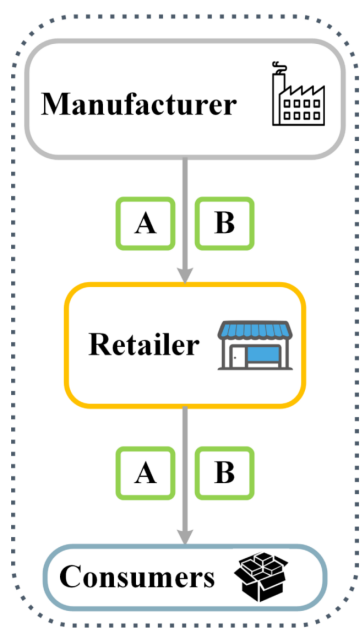

MNRB

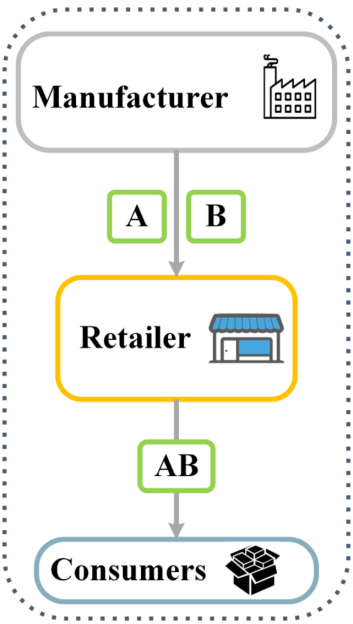

MBRB

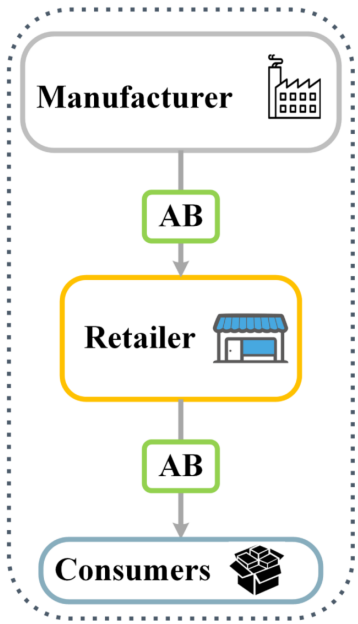

FIgURE 4. Three scenarios composed of different bundling decisions within the supply network.

Based on these best responses, the optimal wholesale prices are:

$$
\begin{aligned}
w_{k i}^{*}= & {\left[8 \beta^{5}\left(35 \alpha+\left(74 c_{k i}+17 c_{k, 3-i}-10 c_{\{A, B\} \backslash k, i}-25 c_{\{A, B\} \backslash k, 3-i}\right) \beta\right)\right.} \\
& -56 \beta^{4}\left(39 \alpha+2\left(55 c_{k i}+11 c_{k, 3-i}-7 c_{\{A, B\} \backslash k, i}-20 c_{\{A, B\} \backslash k, 3-i}\right) \beta\right) \gamma \\
& +4 \beta^{3}\left(1566 \alpha+\left(6295 c_{k i}+998 c_{k, 3-i}-703 c_{\{A, B\} \backslash k, i}-2501 c_{\{A, B\} \backslash k, 3-i}\right) \beta\right) \gamma^{2} \\
& +\beta^{2}\left(-7767 \alpha+\left(-50636 c_{k i}-4801 c_{k, 3-i}+4028 c_{\{A, B\} \backslash k, i}+22531 c_{\{A, B\} \backslash k, 3-i}\right) \beta\right) \gamma^{3} \\
& +9 \beta\left(372 \alpha+\left(5630 c_{k i}-104 c_{k, 3-i}-28 c_{\{A, B\} \backslash k, i}-2933 c_{\{A, B\} \backslash k, 3-i}\right) \beta\right) \gamma^{4} \\
& +9\left(27 \alpha+\left(-2400 c_{k i}+675 c_{k, 3-i}-524 c_{\{A, B\} \backslash k, i}+1613 c_{\{A, B\} \backslash k, 3-i}\right) \beta\right) \gamma^{5} \\
& \left.+9\left(218 c_{k i}-350 c_{k, 3-i}+360 c_{\{A, B\} \backslash k, i}-279 c_{\{A, B\} \backslash k, 3-i}\right) \gamma^{6}\right] \\
& /\left[3\left(12 \beta^{3}-70 \beta^{2} \gamma+127 \beta \gamma^{2}-71 \gamma^{3}\right)\left(28 \beta^{3}-126 \beta^{2} \gamma+135 \beta \gamma^{2}+9 \gamma^{3}\right)\right] .
\end{aligned}
$$

Case MBRB in Figure 4, considers bundling for both layers of supply network (manufacturing and retailing layer in Fig. 2). In such setting, the profit functions will be given by equation (3.3). The best response product and bundle retail prices are:

$$
\begin{aligned}
p_{k i}^{*}(\vec{w})= & \frac{1}{15}\left(2 v_{i i}-3 v_{3-i, 3-i}+10 w_{k i}-5 w_{\{A, B\} \backslash k, i}\right. \\
& \left.+\frac{10 \alpha+\left(v_{i i}+v_{3-i, 3-i}\right) \beta}{2 \beta-5 \gamma}-\frac{5\left(v_{i i}-v_{3-i, 3-i}\right) \beta}{2 \beta-3 \gamma}\right), \\
s_{i i}^{b *}(\vec{w})= & \frac{\alpha}{(2 \beta-5 \gamma)}+\frac{(\beta-2 \gamma)\left(2 v_{i i}(\beta-2 \gamma)+v_{3-i, 3-i} \gamma\right)}{(2 \beta-5 \gamma)(2 \beta-3 \gamma)} .
\end{aligned}
$$

Interestingly, the product prices are dependent on bundle wholesale prices and the cross-product price from the other manufacturer while the best response bundle retail prices are only dependent on the bundle wholesale 
prices. The final optimal set of prices for manufacturers are:

$$
\begin{aligned}
w_{k i}^{*}= & {\left[\left(16 \beta^{3}\left(\alpha+2 c_{k i} \beta-c_{\{A, B\} \backslash k, 3-i} \beta\right)\right.\right.} \\
& -8 \beta^{2}\left(11 \alpha+\left(33 c_{k i}-2 c_{k, 3-i}+c_{\{A, B\} \backslash k, i}-18 c_{\{A, B\} \backslash k, 3-i}\right) \beta\right) \gamma \\
& +\beta\left(153 \alpha+\left(790 c_{k i}-106 c_{k, 3-i}+56 c_{\{A, B\} \backslash k, i}-473 c_{\{A, B\} \backslash k, 3-i}\right) \beta\right) \gamma^{2} \\
& -3\left(28 \alpha+\left(338 c_{k i}-75 c_{k, 3-i}+42 c_{\{A, B\} \backslash k, i}-223 c_{\{A, B\} \backslash k, 3-i}\right) \beta\right) \gamma^{3} \\
& \left.\left.+6\left(79 c_{k i}-25 c_{k, 3-i}+15 c_{\{A, B\} \backslash k, i}-57 c_{\{A, B\} \backslash k, 3-i}\right) \gamma^{4}\right)\right] \\
& /\left[3\left(4 \beta^{2}-15 \beta \gamma+12 \gamma^{2}\right)\left(4 \beta^{2}-17 \beta \gamma+16 \gamma^{2}\right)\right], \\
v_{i i}^{b *}(\vec{w})= & \alpha(2 \beta-3 \gamma)\left(4 \beta^{2}-15 \beta \gamma+12 \gamma^{2}\right)+\left(2 \beta^{2}-8 \beta \gamma+7 \gamma^{2}\right)\left(4\left(c_{A i}+c_{B i}\right) \beta^{2}\right. \\
& \left.+\left(-16 c_{A i}+c_{A, 3-i}-16 c_{B i}+c_{B, 3-i}\right) \beta \gamma+2\left(7 c_{A i}-c_{A, 3-i}+7 c_{B i}-c_{B, 3-i}\right) \gamma^{2}\right) \\
& /\left[\left(4 \beta^{2}-15 \beta \gamma+12 \gamma^{2}\right)\left(4 \beta^{2}-17 \beta \gamma+16 \gamma^{2}\right)\right] .
\end{aligned}
$$

\subsection{The retailer leading Bertrand game (RSB)}

Because the demands are functions of retail prices, the wholesale system of equations (such as Eq. (4.9)) returns no feasible solution. Zhao et al. [34] suggest incorporating the wholesale price in the demand function. This is done by adding a positive margin which is enjoyed by the retailer from selling the product. The positive margin acts like a mediator. First we replace the retail price variables by:

$$
p_{k i}=w_{k i}+m_{A i}, \quad \forall k \in\{A, B\} \wedge i \in\{1,2\} .
$$

Then the wholesale prices' best responses are derived in response to earlier known retail prices, which are set by the retailers. These best responses include the new positive margin variable. The rearrangement $m_{A i}=p_{k i}-w_{k i}$ helps forming a new system of equations. Solving it in terms of wholesale prices, we obtain the desired best responses devoid of mediators. Therefore, the structure of the game is:

$$
\begin{aligned}
& \left\{\begin{array}{l}
\max _{p_{A 1}, p_{B 1}} \pi_{R 1}\left(p_{A 1}, p_{B 1}, w_{A 1}^{*}(\vec{p}), w_{B 1}^{*}(\vec{p}), w_{A 2}^{*}(\vec{p}), w_{B 2}^{*}(\vec{p})\right) \\
\max _{p^{2}, p_{B 2}} \pi_{R 2}\left(w_{A 2}, w_{B 2}, w_{A 1}^{*}(\vec{p}), w_{B 1}^{*}(\vec{p}), w_{A 2}^{*}(\vec{p}), w_{B 2}^{*}(\vec{p})\right)
\end{array}\right. \\
& \Leftarrow \stackrel{\left(w_{A 1}^{*}(\vec{p}), w_{B 1}^{*}(\vec{p}), w_{A 2}^{*}(\vec{p}), w_{B 2}^{*}(\vec{p})\right)}{=\text { Solution of }\left\{\forall k \in\{A, B\} \wedge i \in\{1,2\}, w_{k i}=w_{k i}^{*}(\vec{m}) \mid m_{k i}=p_{k i}-w_{k i}\right\}} \\
& \Leftarrow\left\{\begin{array}{l}
\left(w_{A 1}^{*}(\vec{m}), w_{B 1}^{*}(\vec{m})\right)=\underset{\left(w_{A 1}, w_{B 1}\right)}{\arg \max } \pi_{M 1}\left(m_{A 1}, m_{B 1}, w_{A 1}, w_{B 1}\right), \\
\left(w_{A 2}^{*}(\vec{m}), w_{B 2}^{*}(\vec{m})\right)=\underset{\left(w_{A 2}, w_{B 2}\right)}{\arg \max } \pi_{M 2}\left(m_{A 2}, m_{B 2}, w_{A 2}, w_{B 2}\right) .
\end{array}\right.
\end{aligned}
$$

The vector $\vec{p}$ represents quadruple $\left(p_{A 1}, p_{B 1}, p_{A 2}, p_{B 2}\right)$. Appendix B contains the optimal solutions of this case.

\section{Games With simultaneous and Sequential moves}

In this section we consider cases in which there is possibility of sequential pricings by individuals in one and/or both the upstream and downstream of the supply chain. Six game structures can be defined, two of which include simultaneous pricing in one of the supply network layers (see Fig. 5). Here, when manufacturers lead the game, they play Stackelberg together and the retailers follow their decisions in a simultaneous manner. 


\section{RSS-MS}

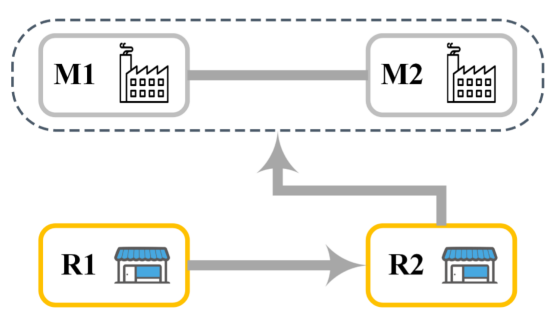

MSS-RS

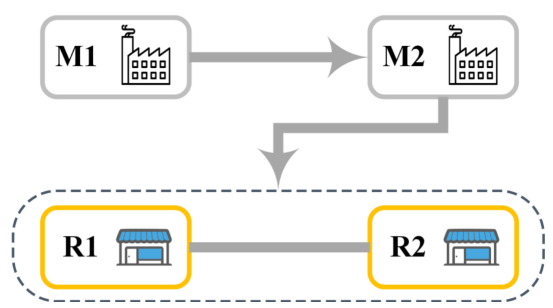

FIgURE 5. The RSS game (left) with manufacturers optimizing profits simultaneously (-MS) and MSS game (right) with retailers optimizing profits simultaneously (-RS).

In the case of the classic MSS game which we call MSS-RS (Fig. 5 - right), the game structure is:

$$
\begin{aligned}
& \left\{\begin{array}{l}
\left(w_{A 1}^{*}, w_{B 1}^{*}\right)=\underset{w_{A 1}, w_{B 1}}{\arg \max } \pi_{M 1}\left(\vec{w}, \vec{p}^{*}(\vec{w})\right), \\
\text { Update } \vec{w}=\left(w_{A 1}, w_{B 1}, w_{A 2}^{*}, w_{B 2}^{*}\right),
\end{array}\right. \\
& \Leftarrow\left\{\begin{array}{l}
\left(w_{A 2}^{*}, w_{B 2}^{*}\right)=\underset{w_{A 2}, w_{B 2}}{\arg \max } \pi_{M 2}\left(\vec{w}, \vec{p}^{*}(\vec{w})\right) \\
\Leftarrow\left\{\begin{array}{l}
\left(p_{A 1}^{*}(\vec{w}), p_{B 1}^{*}(\vec{w})\right)=\underset{\left(p_{A 1}, p_{B 1}\right)}{\arg \max } \pi_{R 1}(\vec{p}, \vec{w}), \\
\left(p_{A 2}^{*}(\vec{w}), p_{B 2}^{*}(\vec{w})\right)=\underset{\left(p_{A 2}, p_{B 2}\right)}{\arg \max } \pi_{R 2}(\vec{p}, \vec{w}) .
\end{array}\right.
\end{array}\right.
\end{aligned}
$$

The vector $\vec{w}$ represents quadruple $\left(w_{A 1}, w_{B 1}, w_{A 2}, w_{B 2}\right)$. The structure in (4.11) indicates that assuming the manufacturers have finished playing Stackelberg, their optimal wholesale prices are determined and now it is time for the retailers to respond to these prices simultaneously. The last action that the retailers can respond to is the wholesale price of the second manufacturer.

Assuming that the retailers (the followers) can also play Stackelberg, we can define four extra structures for the MSS game (see Fig. 6). As an example, the sequential game structure for the case MSSiii is given as follows:

$$
\begin{aligned}
& \left\{\begin{array}{l}
\left(w_{A 1}^{*}, w_{B 1}^{*}\right)=\underset{w_{A 1}, w_{B 1}}{\arg \max } \pi_{M 1}\left(\vec{w}, \vec{p}^{*}(\vec{w})\right), \\
\text { Update } \vec{w}=\left(w_{A 1}, w_{B 1}, w_{A 2}^{*}, w_{B 2}^{*}\right),
\end{array}\right. \\
& \Leftarrow\left\{\begin{array}{l}
\left(w_{A 2}^{*}, w_{B 2}^{*}\right)=\underset{w_{A 2}, w_{B 2}}{\arg \max } \pi_{M 2}\left(\vec{w}, \vec{p}^{*}(\vec{w})\right), \\
\Leftarrow\left\{\begin{array}{l}
\left(p_{A 1}^{*}(\vec{w}), p_{B 1}^{*}(\vec{w})\right)=\underset{\left(p_{A 1}, p_{B 1}\right)}{\arg \max } \pi_{R 1}(\vec{p}, \vec{w}), \\
\operatorname{Update} \vec{p}=\left(p_{A 1}, p_{B 1}, p_{A 2}^{*}, p_{B 2}^{*}\right), \\
\Leftarrow\left(p_{A 2}^{*}(\vec{w}), p_{B 2}^{*}(\vec{w})\right)=\underset{\left(p_{A 2}, p_{B 2}\right)}{\arg \max } \pi_{R 2}(\vec{p}, \vec{w}) .
\end{array}\right.
\end{array}\right.
\end{aligned}
$$

Four sequential scenarios can also be defined for the RSS game, in reverse directions, represented by RSSi, RSSii, RSSiii and RSSiv, respectively (Fig. 7). The process of finding optimal control variables are as in MSSRS and RSS-MS cases. The geometrical structures of the scenarios reveal that each case has one identical configuration (by rotation), only with switching values in the optimal price sets; MSSi and MSSii are identical, also MSSiii and MSSiv.

Therefore, other than the MSS-RS and RSS-MS game structures (Fig. 5), we end up with another four distinct game structures namely MSSi\&ii, MSSiii\&iv, RSSi\&ii and RSSiii\&iv. The solutions of each Stackelberg game structure are congested mathematically and would consume a lot of pages. However, for the readers to capture some elaboration on exact optimal prices, we have provided the solutions of MSS-RS games in Appendix C. All exact solutions of both Bertrand and Stackelberg games can be found in Mathematica (.nb) files provided as supplementary electronic files along with this paper. 
MSS i

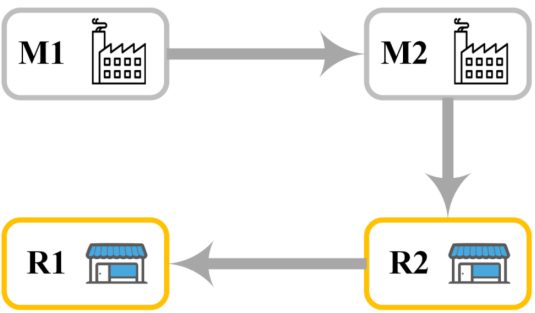

MSS iii

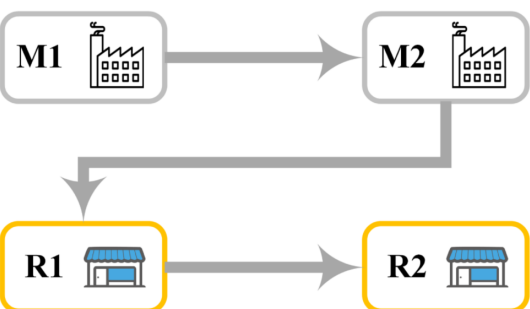

MSS ii

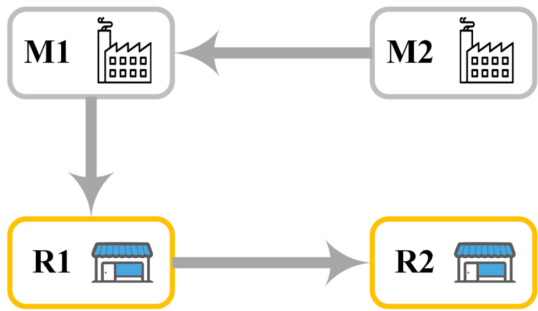

MSS iv

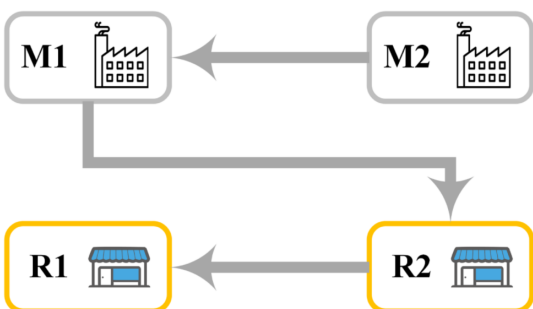

FiguRE 6. Four scenarios of individuals' sequential pricing in the MSS game; the arrows represent sequence ordering, not relations.

RSS i

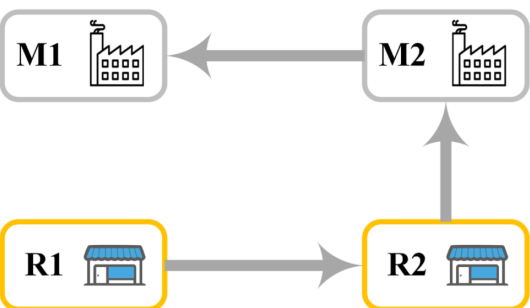

RSS iii

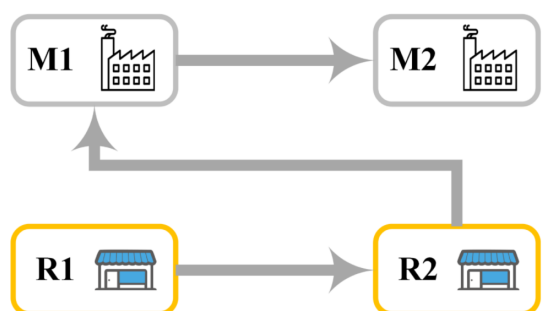

RSS ii

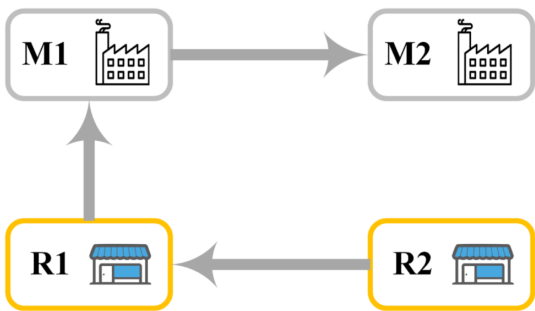

RSS iv

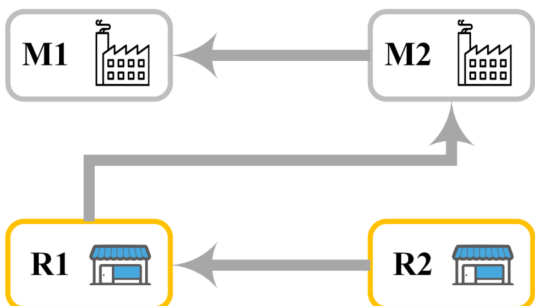

FIGURE 7. Four scenarios of individuals' sequential pricing in the RSS game; the arrows represent sequence ordering, not relations. 


\section{Comparative ANALYSis AND MANAGERIAL IMPLiCATIONS}

The results discussed in this section can be used by managers and decision makers to either shape a supply network with parallel distribution channels and bundling opportunities or devise future plans in an already configured supply network with known levels of its players' market powers.

First we accomplish mathematical and parametric analysis in Section 6.1 to ensure the strict reliability of the insights. We perform a comparative analysis is performed on the impacts of asymmetric costs on prices and demands. Hence the insights will also hold true for symmetric settings. The sensitivity analysis assumes a new concept of cost reduction, along with a compensation that covers the consequences a cost reduction might have. Section 6.2 provides a comparative analysis on the distances between different optimal prices. Section 6.3 specifically focuses on profit changes and how different players tendencies are based on this criteria. Under numerical experiments, Section 6.4 analyzes the behavior of game models under different bundling strategies.

\subsection{Sensitivity analyses on asymmetric costs}

The solutions show a significant presence of the manufacturing costs in determining optimal outcomes. A symmetric setting will mitigate the complexity of the model and also provide some relevant insights on when there is identical conditions. However, it will ignore any possible maneuvering for the players on their endogenous control variables (e.g. manufacturing cost). Therefore, asymmetry is essential to capture the useful insights of the game structures discussed in this article. Here the market parameters $\alpha, \beta$ and $\gamma$ are assumed to be exogenous and thus not controllable by either manufacturers or retailers.

We justify the cost changing scenarios by considering the concept of cost reduction. This way the results can provide better insights for decision makers who are seeking to see whether the trouble of cost reduction is worth it and what it will do to the optimal structure. In order to make this assumption more realistic we also have to consider the fact that cost reduction itself incurs cost. Instead of adding additional parameters to the model such as research and development costs which result in cost reduction, we normalize those costs into the other product's manufacturing cost by the same magnitude. This helps compensate the cost reduction that has just occurred. Therefore, the concept is named cost reduction with compensation (CRC).

This assumption is mathematically equivalent to assuming that the expressions $c_{A 1}+c_{B 1}+c_{A 2}+c_{B 2}$ and $c_{A 1}+c_{B 1}-c_{A 2}-c_{B 2}$ remain constant through any cost reduction activity. For instance, when $c_{B 1}$ is reduced by $\delta$, the cost for that reduction is compensated by adding $\delta$ units to $c_{A 1}$, that concludes $\left(c_{A 1}+\delta\right)+\left(c_{B 1}-\delta\right)=c_{A 1}+c_{B 1}$. Thus both of the above expressions remain constant. It works the other way too and also for when both manufacturers practice cost reduction. The two expressions appear in most of the optimal equations. The price and demand solutions are all linear in terms of costs; therefore, by some rearrangements, the above expressions are cornered with their corresponding coefficients, leaving behind a linear combination of costs, which indeed determines the impact of cost reduction.

Proposition 6.1. Consider optimal retail price $p_{k i}(k \in\{A, B\} \wedge i \in\{1,2\})$. Let $\varphi_{k i}^{A 1}, \varphi_{k i}^{B 1}, \varphi_{k i}^{A 2}$ and $\varphi_{k i}^{B 2}$ be the coefficients of $c_{A 1}, c_{B 1}, c_{A 2}$ and $c_{B 2}$, respectively, in the leftovers of $p_{k i}$, after putting aside all the expressions in $p_{k i}$ which are either independent of any manufacturing costs, or in terms of the expressions $\sum_{n \in\{A, B\}} c_{n j}$, and $c_{k i}+c_{k, 3-i}-c_{\{A, B\} \backslash k, i}-c_{\{A, B\} \backslash k, 3-i}$. In that case, when manufacturer 1 practices $\delta$ units of cost reduction for product $k i$, the change in that corresponding optimal retail price is equivalent to $\delta\left(\varphi_{k i}^{\{A, B\} \backslash k, i}-\varphi_{k i}^{k i}\right)$. The same result holds for optimal wholesale prices.

Proof of Proposition 6.1 can be found in Appendix A.3. Manufacturer 1 and 2's cost reduction practices with compensation (which are, w.l.o.g, considered by $\delta=1$ unit of reduction in product $B$ 's cost) are shown by $\operatorname{CRC}(B)$ for columns $M 1$ and $M 2$, respectively. Table 3 shows the changes in prices and demands based on cost reductons practiced by both manufacturers, for game structures MSB and RSB. Table 4 shows the results for $\operatorname{M1CRC}(B)$, for the other game structures. The results corresponding to $\mathrm{M} 2 \mathrm{CRC}(B)$ of these games can 
TABle 3. Differences in optimal prices and demands due to CRCs in Bertrand games $(\delta=1)$.

\begin{tabular}{lllll}
\hline \hline GS & \multicolumn{2}{c}{ MSB } & \multicolumn{2}{c}{ RSB } \\
\hline CRC $(B 1)$ & \multicolumn{1}{c}{$M 1$} & $M 2$ & $M 1$ & \multicolumn{1}{c}{$M 2$} \\
\hline$\Delta\left(p_{A 1}, w_{A 1}\right)$ & $+(5 / 9,2 / 3)$ & $+(4 / 9,1 / 3)$ & $+(4 / 7,6 / 7)$ & $+(3 / 7,1 / 7)$ \\
$\Delta\left(p_{B 1}, w_{B 1}\right)$ & $-(5 / 9,2 / 3)$ & $-(4 / 9,1 / 3)$ & $-(4 / 7,6 / 7)$ & $-(3 / 7,1 / 7)$ \\
$\Delta\left(p_{A 2}, w_{A 2}\right)$ & $+(4 / 9,1 / 3)$ & $+(5 / 9,2 / 3)$ & $+(3 / 7,1 / 7)$ & $+(4 / 7,6 / 7)$ \\
$\Delta\left(p_{B 2}, w_{B 2}\right)$ & $-(4 / 9,1 / 3)$ & $-(5 / 9,2 / 3)$ & $-(3 / 7,1 / 7)$ & $-(4 / 7,6 / 7)$ \\
$\Delta D_{11} /(\beta+\gamma)$ & 0 & 0 & 0 & 0 \\
$\Delta D_{12} /(\beta+\gamma)$ & $-1 / 9$ & $+1 / 9$ & $-1 / 7$ & $+1 / 7$ \\
$\Delta D_{21} /(\beta+\gamma)$ & $+1 / 9$ & $-1 / 9$ & $+1 / 7$ & $-1 / 7$ \\
$\Delta D_{22} /(\beta+\gamma)$ & 0 & 0 & 0 & 0 \\
\hline
\end{tabular}

TABle 4. Differences in optimal prices, demands and profits due to CRCs in Stackelberg games $(\operatorname{M1CRC}(B)$ and $\delta=1)$.

\begin{tabular}{llll}
\hline \hline GS & MSS - RS & MSS - i\&ii & MSS - iii\&iv \\
\hline$\Delta\left(p_{A 1}, w_{A 1}\right)$ & $+(5 / 12,1 / 2)$ & $+(7 / 16,1 / 2)$ & $+(3 / 8,1 / 2)$ \\
$\Delta\left(p_{B 1}, w_{B 1}\right)$ & $-(5 / 12,1 / 2)$ & $-(7 / 16,1 / 2)$ & $-(3 / 8,1 / 2)$ \\
$\Delta\left(p_{A 2}, w_{A 2}\right)$ & $+(1 / 3,1 / 4)$ & $+(3 / 8,1 / 4)$ & $+(5 / 16,1 / 4)$ \\
$\Delta\left(p_{B 2}, w_{B 2}\right)$ & $-(1 / 3,1 / 4)$ & $-(3 / 8,1 / 4)$ & $-(5 / 16,1 / 4)$ \\
$\Delta D_{11} /(\beta+\gamma)$ & 0 & 0 & 0 \\
$\Delta D_{12} /(\beta+\gamma)$ & $-1 / 12$ & $-1 / 16$ & $-1 / 16$ \\
$\Delta D_{21} /(\beta+\gamma)$ & $+1 / 12$ & $+1 / 16$ & $+1 / 16$ \\
$\Delta D_{22} /(\beta+\gamma)$ & 0 & 0 & 0 \\
\hline $\mathrm{GS}$ & $\mathrm{RSS}-\mathrm{MS}$ & $\mathrm{RSS}-\mathrm{i} \& \mathrm{ii}$ & $\mathrm{RSS}-$ iii\&iv \\
\hline$\Delta\left(p_{A 1}, w_{A 1}\right)$ & $+(2 / 5,9 / 10)$ & $+(3 / 7,13 / 14)$ & $+(1 / 3,5 / 6)$ \\
$\Delta\left(p_{B 1}, w_{B 1}\right)$ & $-(2 / 5,9 / 10)$ & $-(3 / 7,13 / 14)$ & $-(1 / 3,5 / 6)$ \\
$\Delta\left(p_{A 2}, w_{A 2}\right)$ & $+(3 / 10,1 / 10)$ & $+(5 / 14,1 / 7)$ & $+(1 / 4,1 / 12)$ \\
$\Delta\left(p_{B 2}, w_{B 2}\right)$ & $-(3 / 10,1 / 10)$ & $-(5 / 14,1 / 7)$ & $-(1 / 4,1 / 12)$ \\
$\Delta D_{11} /(\beta+\gamma)$ & 0 & 0 & 0 \\
$\Delta D_{12} /(\beta+\gamma)$ & $-1 / 10$ & $-1 / 14$ & $-1 / 12$ \\
$\Delta D_{21} /(\beta+\gamma)$ & $+1 / 10$ & $+1 / 14$ & $+1 / 12$ \\
$\Delta D_{22} /(\beta+\gamma)$ & 0 & 0 & 0 \\
\hline
\end{tabular}

also be obtained due to symmetry observed in Table 3. Now that price changes are given, we calculate demand changes as shown in Appendix A.3. All the numbers in Tables 3 and 4 are derived based on Proposition 6.1.

Corollary 6.2. When in parallel distribution channels within a supply network, the results of applying CRC are different for different game structures. However, for a specific game structure, different bundling strategies (MNRN, MNRB or MBRB) behave the same on price changes, and thus also demand changes when applying $C R C$.

Corollary 6.2 follows by Proposition 6.1 and Tables 3 and 4. The indifference of cost reduction impact among bundling decisions is basically due to the linearity of demand functions and the fact that optimal bundle prices do not experience any change. In transition from no bundling to bundling strategies, new variables are added to the model but because they are involved in a linear arena with previous variables, the obsolete optimal outcomes play some sort of give and take together and their values will be distributed in a homogenous manner. Therefore, in general we observe no change in $\Delta \mathrm{s}$. 
Let $\left.D_{k,\{A, B\} \backslash k}\right|_{\mathrm{M} i \mathrm{CRC}(k)}$ be the demand of consumers who buy product $k$ from manufacturer 1 and product $\{A, B\} \backslash k$ from manufacturer 2 , under a cost reduction on product $k$ with compensation performed by manufacturer $i$.

Corollary 6.3. Demands of direct composites (bundles of separate purchases consisted of complementary components from the same retailer) do not change due to any CRCs, no matter whether it is performed on one's own channel products or on the other channel's. However, the indirect composites' demands will change and the following relations always hold: $\left.D_{k,\{A, B\} \backslash k}\right|_{\mathrm{M} 1 \mathrm{CRC}(k)}=-\left.D_{k,\{A, B\} \backslash k}\right|_{\mathrm{M} 2 \mathrm{CRC}(k)},\left.D_{k,\{A, B\} \backslash k}\right|_{\mathrm{M} i \mathrm{CRC}(k)}>0$ and $\left.D_{\{A, B\} \backslash k, k}\right|_{\mathrm{MiCRC}(k)}>0$. The result holds for all game structures, and within each structure the values do not change due to different bundling strategies. From these results, we also conclude that the market size does not change due to any applied CRC strategy.

Corollary 6.3, followed by Proposition 6.1 and Tables 3 and 4, will come in handy later when evaluating profit changes in Section 6.3.

Corollary 6.4. Due to an individual cost reduction on product $k$ of manufacturer $i$ ( $k \in\{1,2\} \wedge i \in\{1,2\}$ ) compensated by cost increase of product $\{A, B\} \backslash k$ of manufacturer $i$, the retail and wholesale prices change in the following order:

$$
\begin{aligned}
& \Delta^{+} w_{k, 3-i}<\Delta^{+} p_{k, 3-i}<\Delta^{+} p_{k i}<\Delta^{+} w_{k i}, \\
& \Delta^{-} w_{\{A, B\} \backslash k, 3-i}<\Delta^{-} p_{\{A, B\} \backslash k, 3-i}<\Delta^{-} p_{\{A, B\} \backslash k i}<\Delta^{-} w_{\{A, B\} \backslash k, i} .
\end{aligned}
$$

The price change values are all considered positive in the above inequalities, while the signs on $\Delta^{+}$and $\Delta^{-}$ mean increasing and decreasing change, respectively. The inequalities reveal that a change in a product's cost mostly affects its wholesale price and then its retail price, which seems clear. The interesting insight is that the substitute's retail price changes more than its wholesale price. A same discussion holds for their complementary products. This result holds for all game structures and bundling strategies, and also for every $k \in\{1,2\}$ and $i \in\{1,2\}$ we have $\left(\Delta^{-} w_{\{A, B\} \backslash k, i}, \Delta^{-} p_{\{A, B\} \backslash k, i}\right)=\left(\Delta^{+} w_{k, i}, \Delta^{+} p_{k, i}\right)$.

For all the manufacturer leading games, the differences between consecutive price changes in the above ordering are equal. This is probably due to the fact that in these game structures the more powerful players are also the direct handlers of manufacturing costs. For instance, for MSSi\&ii, the differences (divided by $\beta+\gamma$ ) are equal to $1 / 8=1 / 2-7 / 16=7 / 16-3 / 8=3 / 8-1 / 4$, while for RSS-MS the differences are $3 / 7=6 / 7-4 / 7$, $1 / 7=4 / 7-3 / 7$, and $2 / 7=3 / 7-1 / 7$.

The optimal prices have many similar expressions. This is due to the simultaneous moves in the game structures. However, the manufacturing costs coefficients are indeed different among them. In order to understand the sensitivity of each optimal outcome to a relevant unit manufacturing cost, we shall also consider what happens inside these coefficients.

Let $C_{p_{k i}^{*}}$, be the part of optimal retail price $p_{k i}^{*}$, which is either independent of any manufacturing costs, or in terms of the expressions $\sum_{n \in\{A, B\}} \sum_{j \in\{1,2\}} c_{n j}$ (simply $c_{A 1}+c_{B 1}+c_{A 2}+c_{B 2}$ ), and $c_{k i}+c_{k, 3-i}-c_{\{A, B\} \backslash k, i}-$ $c_{\{A, B\} \backslash k, 3-i}$ (e.g. for manufacturer 1, this expression represents $c_{A 1}+c_{B 1}-c_{A 2}-c_{B 2}$ ). Now let $\varphi_{k i}^{m j}$ be the coefficient of $c_{m j}$ in $p_{k i}^{*}-C_{p_{k i}^{*}}$ (cf. Appendix A.3). Let $\psi^{1} \mid \varphi_{k i}^{m j}$ be the expression in the coefficient $\varphi_{k i}^{m j}$ which is dependent on $\beta$ and $\gamma$, and $\psi^{\circ} \mid \varphi_{k i}^{m j}$ be the independent expression. One might expect that for game structures where there are simultaneous moves there would be equal values for $\psi^{1} \mid \varphi_{k i}^{m j}$ s or even $\psi^{\circ} \mid \varphi_{k i}^{m j}$ s. Surprisingly, that is not the case here. The main reason of this difference, is the existence of market power.

Proposition 6.5. $\forall k, i$, no matter what the game structure is, $\forall j \in\{1,2\}$, the $\psi^{1} \mid \varphi_{k i}^{m j} s(\forall m \in\{A, B\})$ are equal, while $\psi^{\circ} \mid \varphi_{k i}^{m j} s$ are different. Also, the $\psi^{1} \mid \varphi_{k i}^{m j} s$ are different $\forall j s$.

Unlike the results in the cost reduction scenarios, here the results are not exactly the same for other bundling strategies of a specific game structure. For brevity, we have just discussed the resulting corollaries for no bundling case (MNRN). See Appendix A.4 for examples of how Proposition 6.5 is established. 
TABLE 5. The independent expressions in cost coefficients of prices in Bertrand games; scenario MNRN.

\begin{tabular}{lrrrrrrrr}
\hline \hline GS & \multicolumn{9}{c}{ MSB } & \multicolumn{4}{c}{$\mathrm{RSB}$} \\
\hline$\psi^{\circ} \mid \varphi$ & \multicolumn{1}{c}{$c_{A 1}$} & \multicolumn{1}{c}{$c_{B 1}$} & \multicolumn{1}{c}{$c_{A 2}$} & \multicolumn{1}{c}{$c_{B 2}$} & \multicolumn{1}{c}{$c_{A 1}$} & \multicolumn{1}{c}{$c_{B 1}$} & \multicolumn{1}{c}{$c_{A 2}$} & \multicolumn{1}{c}{$c_{B 2}$} \\
\hline$p_{A 1}$ & 0.267 & -0.288 & 0.237 & -0.208 & -0.934 & -1.505 & 1.495 & 1.066 \\
$p_{B 1}$ & -0.288 & 0.267 & -0.208 & 0.237 & -1.505 & -0.934 & 1.066 & 1.495 \\
$p_{A 2}$ & 0.237 & -0.208 & 0.267 & -0.288 & 1.495 & 1.066 & -0.934 & -1.505 \\
$p_{B 2}$ & -0.208 & 0.237 & -0.288 & 0.267 & 1.066 & 1.495 & -1.505 & -0.934 \\
$w_{A 1}$ & 0.333 & -0.334 & 0.181 & -0.152 & 0.355 & -0.502 & 0.498 & 0.355 \\
$w_{B 1}$ & -0.334 & 0.333 & -0.152 & 0.181 & -0.502 & 0.355 & 0.355 & 0.498 \\
$w_{A 2}$ & 0.181 & -0.152 & 0.333 & -0.334 & 0.498 & 0.355 & 0.355 & -0.502 \\
$w_{B 2}$ & -0.152 & 0.181 & -0.334 & 0.333 & 0.355 & 0.498 & -0.502 & 0.355 \\
\hline
\end{tabular}

TABLE 6. The independent expressions in cost coefficients of prices in Stackelberg games; scenario MNRN.

\begin{tabular}{lrrrrrrrr}
\hline \hline GS & \multicolumn{9}{c}{ MSS-RS } \\
\hline$\psi^{\circ} \mid \varphi$ & \multicolumn{1}{c}{$c_{A 1}$} & \multicolumn{1}{c}{$c_{B 1}$} & \multicolumn{1}{c}{$c_{A 2}$} & \multicolumn{1}{c}{$c_{B 2}$} & \multicolumn{1}{c}{$c_{A 1}$} & \multicolumn{1}{c}{$c_{B 1}$} & \multicolumn{1}{c}{$c_{A 2}$} & \multicolumn{1}{c}{$c_{B 2}$} \\
\hline$p_{A 1}$ & 3.456 & 3.039 & 0.213 & -0.370 & 0.672 & 0.272 & 0.321 & -0.279 \\
$p_{B 1}$ & 3.039 & 3.456 & -0.370 & 0.213 & 0.272 & 0.672 & -0.279 & 0.321 \\
$p_{A 2}$ & -4.294 & -4.627 & 0.451 & -0.216 & -0.345 & -0.645 & 0.453 & -0.247 \\
$p_{B 2}$ & -4.627 & -4.294 & -0.216 & 0.451 & -0.645 & -0.345 & -0.247 & 0.453 \\
$w_{A 1}$ & 0.500 & 0.000 & 0.500 & 0.000 & 0.900 & 0.000 & 0.100 & 0.000 \\
$w_{B 1}$ & 0.000 & 0.500 & 0.000 & 0.500 & 0.000 & 0.900 & 0.000 & 0.100 \\
$w_{A 2}$ & -4.250 & -4.500 & 0.500 & -0.250 & -0.115 & -0.215 & 0.818 & -0.082 \\
$w_{B 2}$ & -4.500 & -4.250 & -0.250 & 0.500 & -0.215 & -0.115 & -0.082 & 0.818 \\
\hline
\end{tabular}

The reason that we are seeking to drive $\beta$ and $\gamma$ - dependent expressions into a corner and only compare the independent expressions is that comparing the expressions when $\psi^{1} \mid \varphi_{k i}^{m j}$ s are equal is a lot easier because the insights can be derived no matter what the values of the exogenous parameters are. Tables 5 and 6 show the values of $\psi^{\circ} \mid \varphi_{k i}^{m j}$ s for the game structures in which there is at least one simultaneous move. Based on what was concluded on $\psi^{1} \mid \varphi_{k i}^{m j}$ s being different for different $j$ s, notice that when comparing the impact of individual cost changes on these prices it is only meaningful to compare the first two columns ( $\psi^{\circ}$ of $c_{A 1}$ and $c_{B 1}$ ) separately together, and the second two columns $\left(\psi^{\circ}\right.$ of $c_{A 2}$ and $c_{B 2}$ ) together. For better comparative comprehension, the numbers in Tables 5 and 6 are prvided as decimal approximates of the exact rational numbers extracted from the exact mathematical expressions of optimal prices; see Corollaries 6.6 to 6.8 for the comparisons.

Corollary 6.6. In optimal states of Bertrand manufacturer leading game (MSB).

- The increasing effect of an increase in a product's cost on its own (substitute's) retail price is lower than its decreasing effect on its direct (indirect) complementary product's retail price.

- The increasing effect of an increase in a product's cost on its own (substitute's) wholesale price is slightly lower (higher) than its decreasing effect on its direct (indirect) complementary product's wholesale price.

Corollary 6.7. In optimal states of Bertrand retailer leading game (RSB).

- The decreasing (increasing) effect of an increase in a product's cost on its own (substitute's) retail price is lower (higher) than its decreasing (increasing) effect on its direct (indirect) complementary product's retail price. 
TABLE 7 . The coefficient of $\left(c_{B, i}-c_{A, i}\right)-\left(c_{B, 3-i}-c_{A, 3-i}\right)$ in $\left(p_{B, i}-w_{B, i}\right)-\left(p_{A, 3-i}-w_{A, 3-i}\right)$ for different channels $(\mathrm{CH})$ in different game structures.

\begin{tabular}{lllllllll}
\hline \hline & MSB & RSB & MSSRS & MSSi\&ii & MSSiii\&iv & RSSMS & RSSi\&ii & RSSiii\&iv \\
\hline $\mathrm{CH} 1(\zeta)$ & $1 / 9$ & $2 / 7$ & $1 / 12$ & $1 / 16$ & $1 / 8$ & $1 / 2$ & $1 / 2$ & $1 / 2$ \\
$\mathrm{CH} 2(\zeta)$ & $1 / 9$ & $2 / 7$ & $1 / 12$ & $1 / 8$ & $1 / 16$ & $1 / 5$ & $3 / 14$ & $1 / 6$ \\
\hline
\end{tabular}

- The increasing effect of an increase in a product's cost on its own (substitute's) wholesale price is lower (higher) than its decreasing (increasing) effect on its direct (indirect) complementary product's wholesale price.

Corollary 6.8. In optimal states of MSS-RS and RSS-MS games.

- The increasing (decreasing) effect of an increase in a product's cost on its own (substitute's) retail price is higher (lower) than its increasing (decreasing) effect on its direct (indirect) complementary product's retail price.

- An increase in a product's cost only results in an increase in its own wholesale prices and does not affect its direct complementary product's wholesale price.

- The decreasing effect of an increase in a product's cost on its substitute's wholesale price is slightly lower than its decreasing effect on its indirect complementary product's wholesale price.

For example, one unit of increase in $c_{A 1}$ increases $p_{A 1}$ and $p_{B 1}$ by 3.456 and 3.039 , respectively $(3.456>3.039)$, while decreases $p_{A 2}$ and $p_{B 2}$ by 4.294 and 4.627 , respectively $(4.294<4.627)$.

\subsection{On differences between retail and wholesale prices}

The optimal differences $p-w$ are actually complicated with varying coefficients of linear combinations of costs and nonlinear expressions of exogenous parameters. The parallel structure of the supply network again provides us with the advantage of making this analysis easier. Cost asymmetry makes the analysis of $p_{k i}-w_{k i}$ alone sophisticated. However, Proposition 6.5 sheds light on the similarities and differences of cost coefficients within the expressions $p_{k i}-p_{\{A, B\} \backslash k, i}$ and $w_{k i}-w_{\{A, B\} \backslash k, i}$. This triggers the analysis of $p_{k, i}-w_{k, i}-\left(p_{\{A, B\} \backslash k, i}-\right.$ $\left.w_{\{A, B\} \backslash k, i}\right)$.

Proposition 6.9. Regardless of the base market potential $(\alpha)$, price effect $(\beta)$ and degree of substitutability $(\gamma)$, for product $k$ of manufacturer $i$, we have,

$$
p_{k, i}-w_{k, i}-\left(p_{\{A, B\} \backslash k, i}-w_{\{A, B\} \backslash k, i}\right)=\zeta\left[\left(c_{\{A, B\} \backslash k, i}-c_{k, i}\right)-\left(c_{\{A, B\} \backslash k, 3-i}-c_{k, 3-i}\right)\right],
$$

where $\zeta \in \mathbb{Q}^{+}$is a known and different positive rational number for each game structure. In each game structure the value $\zeta$ does not get affected by different bundling strategies. Table 7 shows the values of $\zeta$ for different game structures and because sequential moves can affect it, channels' relevant values are separated.

The right hand side expression in equation (6.2) equals zero at symmetric settings. This reveals that under cost symmetry, we have $p_{k, i}-w_{k, i}=p_{\{A, B\} \backslash k, i}-w_{\{A, B\} \backslash k, i}$. For instance, in RSSMS we have $p_{A 2}-w_{A 2}-$ $\left(p_{B 2}-w_{B 2}\right)=\frac{1}{5}\left[\left(c_{B 2}-c_{A 2}\right)-\left(c_{B 1}-c_{A 1}\right)\right]$.

Assuming, w.l.o.g, that $\left(c_{A 1}-c_{B 1}\right)-\left(c_{A 2}-c_{B 2}\right)>0$ or $c_{A 1}-c_{B 1}>c_{A 2}-c_{B 2}$, we can derive some insights from Table 7 as well. For start, it can be concluded that in every game structure and for each manufacturer, always one of the product's retail price is set higher in regards to its wholesale price than the difference between its complementary product's retail and wholesale price. We call this concept (single product), the retail advantage. For instance, if $c_{A 1}-c_{B 1}>c_{A 2}-c_{B 2}$, the result in Table 7 shows that product channel 2 has a retail 
advantage for product $B$ in MSSiii\&iv meaning if the difference between product $A$ 's optimal retail and wholesale price is a positive value $x$, then retailer 2 is able to set product $B$ 's retail price $x+\frac{1}{16}\left[\left(c_{A 1}-c_{B 1}\right)-\left(c_{A 2}-c_{B 2}\right)\right]$ higher than its wholesale price and as clear the bigger the distance between retail and wholesale price the better for the retailer, and hence the appelation of the phrase retail advantage. The results show that this retail advantage is possible on only one product.

Corollary 6.10. Assuming $c_{A 1}-c_{B 1}>c_{A 2}-c_{B 2}$ and showing absolute values of retail advantage value (excluding the linear combination expression) by $\rho$, we have the following insights and ordinal relations derived from Table 7 ,

- Retail advantage is much higher in retailer leading games than in manufacturer leading games.

- In retail leading games, retail advantage is highest in RSSiEii game followed by other games in the following order,

$$
\rho^{\mathrm{RSSi} i \mathrm{ii}}>\rho^{\mathrm{RSSMS}}>\rho^{\mathrm{RSS} \text { iii\&iv }}>\rho^{\mathrm{RSB}} .
$$

- In manufacturer leading games, retail advantage is highest in MSB game followed by other games in the following order,

$$
\rho^{\mathrm{MSB}}>\rho^{\mathrm{MSSi \& ii}}=\rho^{\mathrm{MSSiii \& iv}}>\rho^{\mathrm{MSSRS}} .
$$

Notice that the value of $\rho$ has been calculated by summing up both channels' retail advantage in a game structure. For instance, $\rho^{\text {RSSMS }}=1 / 2+1 / 5=7 / 10$. A similar relation to equation (6.2) can also be concluded for the difference between complementary product's retail prices, and also the difference between their wholesale prices.

Proposition 6.11. Regardless of the base market potential $(\alpha)$, price effect $(\beta)$ and degree of substitutability $(\gamma)$, for product $k$ of manufacturer $i$, we have,

$$
\begin{aligned}
p_{k, i}-p_{\{A, B\} \backslash k, i} & =m_{p}\left(c_{k, i}-c_{\{A, B\} \backslash k, i}\right)+n_{p}\left(c_{k, 3-i}-c_{\{A, B\} \backslash k, 3-i}\right), \\
w_{k, i}-w_{\{A, B\} \backslash k, i} & =m_{w}\left(c_{k, i}-c_{\{A, B\} \backslash k, i}\right)+n_{w}\left(c_{k, 3-i}-c_{\{A, B\} \backslash k, 3-i}\right),
\end{aligned}
$$

where $m_{p}, n_{p}, m_{w}, n_{w}$ are known and different positive rational numbers for each game structure. In each game structure their values (see Tab. 8) do not get affected by different bundling strategies. Also following relations hold,

$$
\begin{aligned}
& m_{p}+n_{p}=m_{w}+n_{w}=1, \\
& m_{w}-m_{p}=n_{p}-n_{w}=\zeta .
\end{aligned}
$$

For instance, in RSSMS we have $p_{A 2}-p_{B 2}=\frac{7}{10}\left(c_{A 2}-c_{B 2}\right)-\frac{3}{10}\left(c_{A 1}-c_{B 1}\right)$, and for the difference between wholesale prices we have $w_{A 2}-w_{B 2}=\frac{9}{10}\left(c_{A 2}-c_{B 2}\right)-\frac{1}{10}\left(c_{A 1}-c_{B 1}\right)$. Equations (6.5) and (6.6) explain how cost asymmetry itself can change the differences in pricing. Equation (6.8) in fact results from the derivation of equation (6.2) by subtracting equation (6.6) from equation (6.5). The readers can also verify this by using the numbers in Tables 7 and 8 .

\subsection{On profit changes}

It was shown in Proposition 6.1 how optimal price and demand changes are evaluated based on cost reduction. With Propositions 6.9 and 6.11, it becomes easier now to evaluate the profit changes after cost reduction, due to the presence of retail and wholesale price subtractions inside the profit functions. When calculating the profit changes, we realize that if only one manufacturer practices cost reduction, the profit change is still a function of the manufacturing costs. However, when both manufacturers practice cost reduction this dependency is eliminated and no change in profits is observed. We call this situation, a concurrent cost reduction $(C C R C)$. 
TABLE 8. The coefficients $m_{p}, m_{w}$ for different channels $(\mathrm{CH})$ in different game structures.

\begin{tabular}{lllllllll}
\hline \hline & MSB & RSB & MSSRS & MSSi\&ii & MSSiii\&iv & RSSMS & RSSi\&ii & RSSiii\&iv \\
\hline $\mathrm{CH} 1\left(m_{p}\right)$ & $5 / 9$ & $4 / 7$ & $5 / 12$ & $7 / 16$ & $3 / 8$ & $2 / 5$ & $3 / 7$ & $1 / 3$ \\
$\mathrm{CH} 1\left(m_{w}\right)$ & $2 / 3$ & $6 / 7$ & $1 / 2$ & $1 / 2$ & $1 / 2$ & $9 / 10$ & $13 / 14$ & $5 / 6$ \\
\hline $\mathrm{CH} 2\left(m_{p}\right)$ & $5 / 9$ & $4 / 7$ & $2 / 3$ & $5 / 8$ & $11 / 16$ & $7 / 10$ & $9 / 14$ & $3 / 4$ \\
$\mathrm{CH} 2\left(m_{w}\right)$ & $2 / 3$ & $6 / 7$ & $3 / 4$ & $3 / 4$ & $3 / 4$ & $9 / 10$ & $6 / 7$ & $11 / 12$ \\
\hline
\end{tabular}

TABle 9. Profit changes in different game structures.

\begin{tabular}{lllll}
\hline \hline$\times \chi_{\mathrm{M} 1 \mathrm{CRC}(B)}(\delta)$ & MSB & \multicolumn{1}{c}{ RSB } & MSSRS & RSSMS \\
\hline$\Delta \pi_{M 1}$ & $\frac{1}{27}$ & $\frac{1}{49}$ & $\frac{1}{24}$ & $\frac{1}{100}$ \\
$\Delta \pi_{M 2}$ & $\frac{1}{27}$ & $\frac{1}{49}$ & $\frac{1}{48}$ & $\frac{1}{100}$ \\
$\Delta \pi_{R 1}$ & $\frac{1}{81}$ & $\frac{2}{49}$ & $\frac{1}{144}$ & $\frac{1}{20}$ \\
$\Delta \pi_{R 2}$ & $\frac{1}{81}$ & $\frac{2}{49}$ & $\frac{1}{144}$ & $\frac{1}{50}$ \\
$\left.\Delta \sum \pi\right|_{\mathrm{CRC}}$ & $\frac{8}{81}$ & $\frac{6}{49}$ & $\frac{11}{144}$ & $\frac{9}{100}$ \\
\hline$\times \chi_{\mathrm{M} 1 \mathrm{CRC}(B)}(\delta)$ & MSSi\&ii & MSSiii\&iv & RSSi\&ii & RSSiii\&iv \\
\hline$\Delta \pi_{M 1}$ & $\frac{1}{32}$ & $\frac{1}{32}$ & $\frac{1}{196}$ & $\frac{1}{72}$ \\
$\Delta \pi_{M 2}$ & $\frac{1}{64}$ & $\frac{1}{64}$ & $\frac{1}{98}$ & $\frac{1}{144}$ \\
$\Delta \pi_{R 1}$ & $\frac{1}{256}$ & $\frac{1}{128}$ & $\frac{1}{28}$ & $\frac{1}{24}$ \\
$\Delta \pi_{R 2}$ & $\frac{1}{128}$ & $\frac{1}{256}$ & $\frac{3}{196}$ & $\frac{1}{72}$ \\
$\left.\Delta \sum \pi\right|_{\mathrm{CRC}}$ & $\frac{15}{256}$ & $\frac{15}{256}$ & $\frac{13}{196}$ & $\frac{11}{144}$ \\
$\left.\Delta \sum \pi\right|_{\mathrm{CCRC}}$ & & & 0 & \\
\hline
\end{tabular}

Proposition 6.12. Due to $\delta$ units of cost reduction in product $k$ by manufacturer $i$, the profit change for each of the players in supply network is a different rational multiple of a fixed expression:

$$
\chi_{\mathrm{M} i \mathrm{CRC}(k)}(\delta)=\delta\left(\left(\left(c_{\{A, B\} \backslash k, i}-c_{k, i}\right)-\left(c_{\{A, B\} \backslash k, 3-i}-c_{k, 3-i}\right)\right)(1+\beta+\gamma)+2 \delta\right) .
$$

If the CCRC is practiced; meaning both manufacturers reduce costs simultaneously, the profits of players experiences no change.

Proof of Proposition 6.12 can be found in Appendix A.7. The rational multiples for all game structures are shown in Table 9. $\left.\Delta \pi\right|_{\mathrm{CCRC}}$ is zero for all game structures. This is due the fact that price and demand changes based on simultaneous cost reductions are exact negatives of each other.

Notice that when we talk about CRC, only one of the manufacturers is practicing cost reduction, and the other manufacturer applies no changes in his/her costs. The $\chi$ value due to CRC from each manufacturer is as shown in equation (6.10).

$$
\begin{aligned}
& \chi_{\mathrm{M} 1 \mathrm{CRC}(B)}(\delta)=\delta\left(\left(\left(c_{A 1}-c_{B 1}\right)-\left(c_{B 2}-c_{A 2}\right)\right)(1+\beta+\gamma)+2 \delta\right) \\
& \chi_{\mathrm{M} 2 \mathrm{CRC}(B)}(\delta)=\delta\left(\left(\left(c_{A 2}-c_{B 2}\right)-\left(c_{B 1}-c_{A 1}\right)\right)(1+\beta+\gamma)+2 \delta\right) .
\end{aligned}
$$

In games MSB, RSB and RSSMS where there is simultaneous pricing inside the manufacturing layer, the profit changes are equivalent for both manufacturers. The manufacturers might wonder who should practice cost reduction, and who should not. Assume the situation as a classic game theoretical model as shown in 
(6.11), where $r$ is the rational multiple calculated in Table 9, $S$ (standing for Stationary) is the strategy of not practicing cost reduction, and $C$ is the strategy to apply cost reduction with compensation.

$$
\left.\begin{array}{ccc}
\multicolumn{4}{c}{\text { Manufacturer } 2} \\
\text { Manufacturer 1 } & & \multicolumn{4}{c}{C} \\
0 & r \chi_{\mathrm{M} 2 \mathrm{CRC}(B)}(\delta) \\
0 & r \chi_{\mathrm{M} 2 \mathrm{CRC}(B)}(\delta) \\
& C & 0 \\
r \chi_{\mathrm{M} 1 \mathrm{CRC}(B)}(\delta) & (\delta) & 0 \\
r \chi_{\mathrm{M} 1 \mathrm{CRC}(B)}
\end{array}\right) .
$$

Now, assume that w.l.o.g, $c_{A 1}-c_{B 1}>c_{A 2}-c_{B 2}>0$. Therefore, $\chi_{\mathrm{M} 1 \mathrm{CRC}(B)}>0>\chi_{\mathrm{M} 1 \mathrm{CRC}(B)}$, and it can be easily concluded that the strategy profile $(C, S)$ is the dominant Nash equilibrium.

Corollary 6.13. Regarding cost reduction, it is in most benefit of the manufacturers, for the manufacturer that has a higher difference among his/her complementary products' manufacturing costs, to practice cost reduction, while the other manufacturer should maintain his/her costs at their stationary level.

This result holds for other game structures too, regradless of whether the rational multiples (thus, profit changes) among manufacturers are equal or not. In other words, if the mnufacturer with lower difference between the manufacturing costs of his/her complementary products applies cost reduction, the profits will drop.

The rational multiples in Table 9 say a lot about how cost reductions affect different players and layers of the supply network. For better interpretation of how the fractions compare to each other, we have depicted these changes in Figures 8 and 9. The numbers in these figures are approximate decimals of their corresponding fractions, and have been scaled to 1000 for better comparison among the gaps between profit changes. Due to Corollary 6.13, we assume that $c_{A 1}-c_{B 1}>c_{A 2}-c_{B 2}$, so the all the profit changes calculated in Table 9 are positive and therefore, we call them profit gains. At first glance, the most profit gain relates to Retailer 1 in the game structure RSSMS. However, in this scenario the manufacturers have a low profit gain compared to many other game structures. This can be due to the fact that in RSSMS, retailers are leaders therefore they proceed with the approach to maximize their own profits.

An interesting ordinal relation can be observed among profit gains. As we distance from the first mover(s), the profit gain decreases. In other words, there is indeed a first mover advantage even in the case where one of the manufacturers applies cost reduction.

For instance, in MSSiii\&iv (for which w.l.o.g scenario MSSiii of Fig. 6 has been investigated), the first mover is manufacturer 1 with 31.3 , next is manufacturer 2 with 15.6, next is retailer 1 with 7.8, and finally retailer 2 with 3.9. The fractions in Table 9 can provide an even better insight on how each player's share on profit changes is deduced. For instance, again in the case of MSSiii\&iv, the profit gets halved at each stage: $\frac{1}{32}$ for M1, then $\frac{1}{2} \times \frac{1}{32}=\frac{1}{64}$ for M2, then $\frac{1}{2} \times \frac{1}{64}=\frac{1}{128}$ for R1, and finally $\frac{1}{2} \times \frac{1}{128}=\frac{1}{256}$ for R2. These ordinal cost reductions are presented in Table 10 .

Corollary 6.14. When manufacturers are the leaders in pricing, the reduction of profit gain among players experiences more intensity in comparison to when the retailers are leaders in that same game structure.

Corollary 6.14 is simply followed by the results in Table 10. In other words, for the whole supply network to benefit from cost reduction activities, it is better for the retailers to be the first movers of pricing. This finding can also be triggered by the fact that the retailers are neared to the end-customers than manufacturers are. Therefore, they have a better sense of the market. Speaking of which, we have also investigated the whole supply network's profit changes and identified the share of manufacturers and retailers, separately, see Figure 10. Surprisingly, the sum of profit changes conforms the same sorting achieved in Table 10.

Corollary 6.15. It is in benefit of the whole supply network to maintain simultaneous moves (cooperative pricings) as much as possible to achieve a bigger cake for all the players to share. 


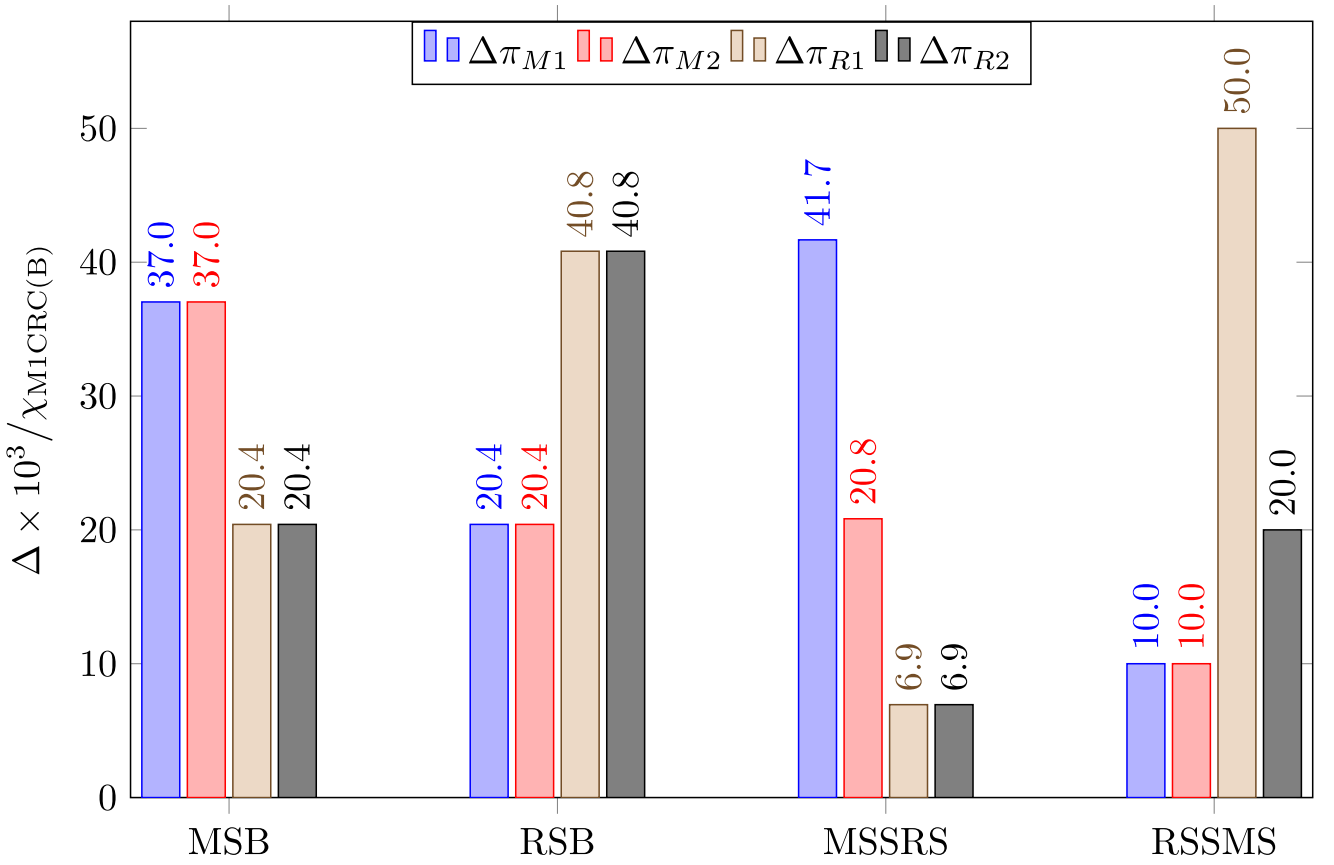

FIGURE 8. Comparing profit changes among all game structures with at least one simultaneous game.

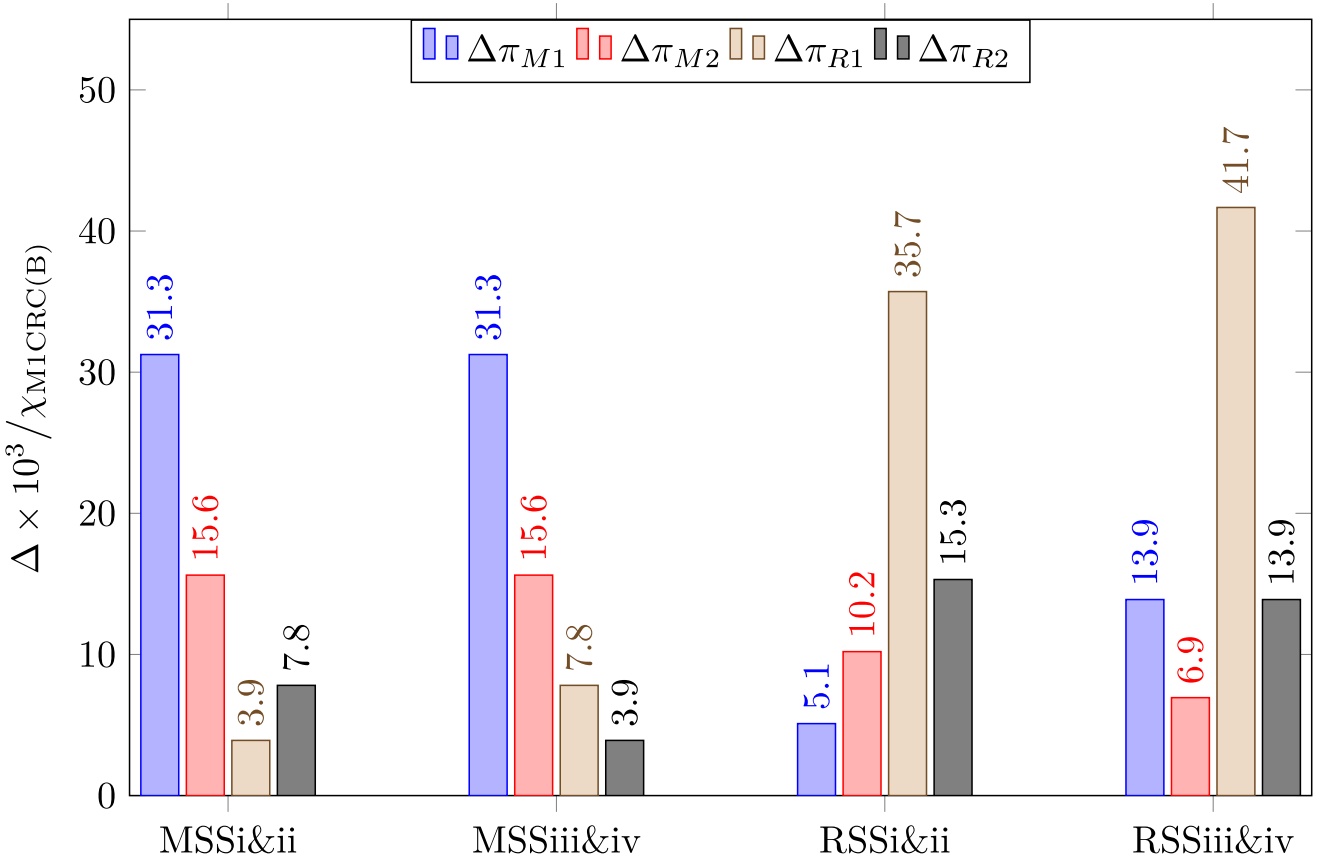

FIGURE 9. Comparing profit changes among all fully sequential game structures. 
TABLE 10. Ordinal cost reduction multiples among all game structures sorted by the reduction from first to last player.

\begin{tabular}{|c|c|c|}
\hline GS & Ordinal reduction & From 1 st to 4 th \\
\hline RSB & $(R 1, R 2) \stackrel{\times 1 / 2}{\rightarrow}(M 1, M 2)$ & $\frac{1}{2}$ \\
\hline MSB & $(M 1, M 2) \stackrel{\times 1 / 3}{\rightarrow}(R 1, R 2)$ & $\frac{1}{3}$ \\
\hline RSSMS & $R 1 \stackrel{\times^{2} / 5}{\rightarrow} R 2 \stackrel{\times^{1 / 2}}{\rightarrow}(M 1, M 2)$ & $\frac{1}{5}$ \\
\hline MSSRS & $M 1 \stackrel{\times^{1 / 2}}{\rightarrow} M 2 \stackrel{\times^{1 / 3}}{\rightarrow}(R 1, R 2)$ & $\frac{1}{6}$ \\
\hline RSSiii\&iv & $R 1 \stackrel{\times 1 / 3}{\rightarrow} R 2 \stackrel{\times 1}{\rightarrow} M 1 \stackrel{\times^{1 / 2}}{\rightarrow} M 2$ & $\frac{1}{6}$ \\
\hline RSSi\&ii & $R 1 \stackrel{\times^{3 /} / 7}{\rightarrow} R 2 \stackrel{\times^{2} / 3}{\rightarrow} M 2 \stackrel{\times^{1 / 2}}{\rightarrow} M 1$ & $\frac{1}{7}$ \\
\hline MSSi\&ii & $M 1 \stackrel{\times 1 / 2}{\rightarrow} M 2 \stackrel{\times 1 / 2}{\rightarrow} R 2 \stackrel{\times 1 / 2}{\rightarrow} R 1$ & $\frac{1}{8}$ \\
\hline MSSiii\&iv & $M 1 \stackrel{\times 1 / 2}{\rightarrow} M 2 \stackrel{\times 1 / 2}{\rightarrow} R 1 \stackrel{\times^{1 / 2}}{\rightarrow} R 2$ & $\frac{1}{8}$ \\
\hline
\end{tabular}

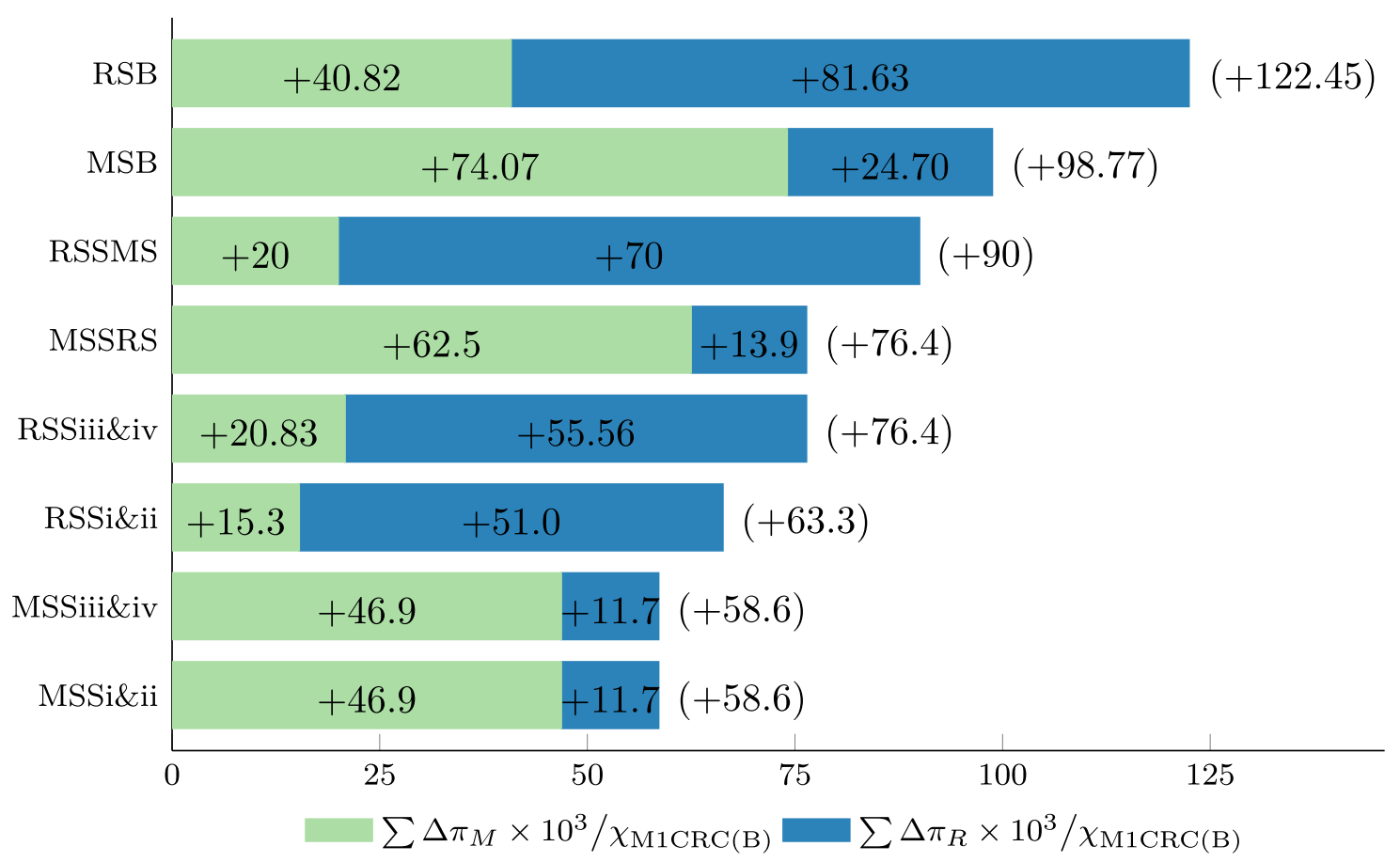

FIGURE 10. Comparing profit gains due to $\operatorname{M} 1 \mathrm{CRC}(B)$ among different game structures within different layers and whole supply network's perspective. 
The results regarding whole supply network, although appealing, are all in favor of retailers. The manufacturers are the ones who actually accept the trouble of cost cutting actions. The results confirm that they prefer a market in which more market power is granted to the manufacturing layer. If we consider the decision of "Whether the manufacturers should set their prices simultaneously or sequentially?", then, the manufacturers' preferences would be as follows.

Corollary 6.16. When the retailing layer has more market power, if the retailers are playing Bertrand, the manufacturers will be sure to choose playing Bertrand as well. The rest of the ordinal preferences of manufacturers in such a case is as follows:

$$
\mathrm{RSB} \succ \operatorname{RSSiii\& iv} \succ \operatorname{RSSMS} \succ \operatorname{RSSi\& ii} .
$$

However, if the manufacturing layer has more market power, the retailers prefer playing Bertrand, no matter the manufacturers have chosen to play Bertrand or Stackelberg. The rest of the ordinal preferences of retailers in such a case is as follows.

$$
\text { MSB } \succ \operatorname{MSSMS} \succ \operatorname{MSSiii\& iv~} \succ \text { MSSi\&ii. }
$$

Corollary 6.16 shows that, when practicing cost reduction, the behavior of retailers and manufacturers will eventually tend to a situation where both layers play Bertrand games, hence promoting cooperation within the channels. Also in fully sequential games, both manufacturers prefer the iii\&iv formation over i\&ii. This means after the last player of a layer has set his/her price, it is preferred that the next pricing action be performed by the player from the opposite channel. For instance in MSSiii, the last player in manufacturing layer to set price is M2, and the next player is R1 from the other channel. Again such a preference can be interpreted as an act of promoting coordination (cooperation) among channels.

\subsection{Numerical experiments on profits, market size and bundling gains}

In some cases a player is forced to choose only one predetermined decision based on previous player's action. In manufacturer leading games, if the manufacturers choose to bundle, the retailers have no choice but to bundle. Otherwise a conflict in demand balance will occur due to our discussion in Section 4. For the same reason, in retailer leading games, if the retailers choose not to bundle then the manufacturers have no other choice than not to bundle as well. Therefore, for the manufacturer leading games, the insights that can be achieved on decisions are whether a retailer should bundle or not bundle in a case manufacturers chooses not to bundle. And for the retailer leading games, insights reveal whether a manufacturer should bundle or not bundle in case retailers choose to bundle.

Looking at the supply network as a whole, there can be two criteria for decision making; chain profit and market size. The former is equal to total profits of firms and the latter is the total demand of all substitutable composites and is used when the players are in the beginning stages of market development thus seek to increase the market size as much as possible. However, it can be concluded that for each specific game structure, the optimal market size remains the same no matter what bundling strategy the players choose. On the other hand, demands of bundles (direct composites) behave differently from demands of indirect composites based on how the players want to act (cooperative or competitive). Therefore, we use the chain profit criterion in this section and analyze the changes in individual demands.

Tables 11 and 12 provide the optimal results of prices, demands and profits for different bundling strategies, then the second mover can choose between due to the above discussion. These numerical results have been derived for $(\alpha, \beta, \gamma)=(8,3,1)$ and $\left(c_{A 1}, c_{B 1}, c_{A 2}, c_{B 2}\right)=(1,1,1,2)$. Notice that $\beta \geq 3 \gamma$ holds and we have applied the lowest possible value for price effect. This means the highest possible value for cross-price effect or in other words the highest degree of substitution. We have also examined the results for a symmetric case of $\left(c_{A 1}, c_{B 1}, c_{A 2}, c_{B 2}\right)=(1,1,1,1)$ and $(\alpha, \beta, \gamma)=(3,3,1)$ and another asymmetric case of $\left(c_{A 1}, c_{B 1}, c_{A 2}, c_{B 2}\right)=$ $(1,1,2,2)$ and $(\alpha, \beta, \gamma)=(1,3,1)$. The derived insights here are for when the degree of substitution is at its highest possible level and are the same for these numerical cases too. The third column under each game 
TABLE 11. Bundling (Not bundling) gain in chain's demand and profit when the manufacturer chooses not to bundle (retailer chooses to bundle) in Bertrand manufacturer (retailer) leading game.

\begin{tabular}{lrrrrrr}
\hline \hline GS & \multicolumn{2}{c}{ MSSRS (Retailers' decision) } & \multicolumn{3}{c}{ RSSMS (Manufacturers' decision) } \\
\hline Decision & MNRB & MNRN & MNRB gain & MBRB & MNRB & MNRB gain \\
\hline$\sum \pi$ & 1319.19 & 1304.97 & +14.22 & 1032.36 & 1038.6 & +6.24 \\
$\sum D$ & 32 & 32 & 0 & 32 & 32 & 0 \\
\hline$\pi M 1$ & 444.17 & 444.17 & 0 & 65.64 & 65.00 & 0.64 \\
$\pi M 2$ & 590.08 & 590.08 & 0 & 101.96 & 212.20 & +110.24 \\
$\pi R 1$ & 81.13 & 74.03 & +7.11 & 328.20 & 333.00 & +4.80 \\
$\pi R 2$ & 203.80 & 196.69 & +7.11 & 536.56 & 428.40 & 108.16 \\
$D_{11}$ & 9.50 & 4.17 & +5.33 & 10.60 & 9.40 & 1.20 \\
$D_{22}$ & 17.17 & 11.83 & +5.33 & 19.80 & 18.60 & 1.20 \\
$D_{12}$ & 2.50 & 7.83 & 5.33 & 0.60 & 1.80 & +1.20 \\
$D_{21}$ & 2.83 & 8.17 & 5.33 & 1.00 & 2.20 & +1.20 \\
\hline
\end{tabular}

TABLE 12. Bundling (Not bundling) gain in chain's demand and profit when the manufacturer chooses not to bundle (retailer chooses to bundle) in Stackelberg manufacturer (retailer) leading game.

\begin{tabular}{lrrrrrr}
\hline \hline GS & \multicolumn{2}{c}{ MSSiii\&iv (Retailers' decision) } & \multicolumn{3}{c}{ RSSiii\&iv (Manufacturers' decision) } \\
\hline Decision & MNRB & MNRN & MNRB gain & MBRB & MNRB & MNRB gain \\
\hline$\sum \pi$ & 1720.36 & 1708.36 & 12 & 1125.42 & 1132.53 & +7.11 \\
$\sum D$ & 32 & 32 & 0 & 32 & 32 & 0 \\
\hline$\pi M 1$ & 495.125 & 495.125 & 0 & 91.166 & 90.278 & -0.889 \\
$\pi M 2$ & 871.563 & 871.563 & 0 & 193.542 & 253.139 & +59.597 \\
$\pi R 1$ & 131.781 & 123.781 & 8 & 273.500 & 278.833 & +5.333 \\
$\pi R 2$ & 221.891 & 217.891 & 4 & 567.208 & 510.278 & -56.930 \\
$D_{11}$ & 9.125 & 3.125 & 6 & 8.833 & 7.500 & -1.333 \\
$D_{22}$ & 18.875 & 12.875 & 6 & 21.833 & 20.500 & -1.333 \\
$D_{12}$ & 1.875 & 7.875 & -6 & 0.500 & 1.833 & +1.333 \\
$D_{21}$ & 2.125 & 8.125 & -6 & 0.833 & 2.167 & +1.333 \\
\hline
\end{tabular}

structure describes the gain of the corresponding bundling structure formed by the second mover's choice. For instance, in RSS-MS game, when the retailer chooses to bundle, if the manufacturer chooses to bundle as well, then the MBRB bundling structure is formed and the whole chain receives 1032.36 units of profit, while if he/she chooses not to bundle, the MNRB structure is formed leading to 1038.6 units of profit. Hence, the MNRB gain of 6.24 is derived. Table 12 shows the same results for one of fully sequential structures of Stackelberg games. The results are the same for other Stackelberg structures.

The following insights are derived from Tables 11 and 12.

Numerical Insight 6.17. For constant values of exogenous parameters, the optimal market size does not change for different game structures and bundling strategies.

Numerical Insight 6.18. Chain profits are higher in Stackelberg structures than Bertrand structures. Therefore, in order to achieve more chain profit, at least one of the players should make their moves sequentially.

Numerical Insight 6.19. For chain profit maximization, no matter what the game structures are, 
- The increasing (decreasing) effect of an increase in a product's cost on its own (substitute's) retail price is higher (lower) than its increasing (decreasing) effect on its direct (indirect) complementary product's retail price.

- In manufacturer (retailer) leading games, when the manufacturers (retailers) choose not to bundle (to bundle), the retailers (manufacturers) choose to bundle (not to bundle).

- In retailer leading games, when the retailers choose to bundle, the manufacturers choose not to bundle.

Insight 6.19 reveals that the MNRB bundling structure works best than the other two structures when the criteria is chain profit maximization. However, in retailer leading games, it always leads to significant loss for one of the retailers. For instance, in the RSSiii\&iv structure, the MNRB strategy is in favor of all players' profits (the slight decrease in M1's profit is negligible) except retailer 2 who experiences a loss of 56.930 units. In this example, the manufacturers receive an overall gain of 58.807, while the retailers receive an overall loss of 51.597. The loss of retailers is lower than the gain of manufacturers. This mitigation of loss might have occurred due to the more market power of retailers.

Numerical Insight 6.20. The MNRB bundling structure is an unstable bundling strategy profile in retailer leading games. Meaning that the retailers (the more powerful players) are reluctant to be the first mover and are eager to wait for the manufacturers to make their move first. The manufacturers, knowing this, are also eager to let the retailers have their turn. This can also be known as the second mover's advantage. Therefore, for this strategy to be chosen a revenue sharing agreement (contractual coordination) seems necessary in order to drive the market to a stable position.

Although the sum of demands (market size) does not change for any of the game structures, but the individual demands change. For instance, in MSSiii\&iv game, MNRB leads to 6 units of increase in demands of direct composites while the same unit of decrease in the indirect composites' demands.

Numerical Insight 6.21. In manufacturer leading games, if the retailers choose to bundle after the manufacturers have chosen not to bundle, then besides from the fact that a better chain profit is achieved, the demand of direct composites experiences a higher level as well, while in retailers leading games the demand of indirect composites increase. The latter and former case also resemble a cooperative and competitive behavior, respectively. In fact, if the channels are willing to encourage consumers to buy direct composites (competitive) they are better off playing a manufacturers leading game.

The above insight reveals that if the manufacturers move first, the chances of the consumers buying both products from the same channel are higher, while if the retailers move first, the consumers will behave more variety seeking. Insights $6.17-6.21$ were derived based on the lowest value for $\beta$. As $\beta$ increases, and w.l.o.g assuming that $\gamma$ remains constant, the retailer leading games insights do not change, but in manufacturer leading games the MNRN strategy gradually becomes superior to MNRB strategy.

Numerical Insight 6.22. As the degree of substitutability gets lower, the retailers become more reluctant to bundle, after observing that the manufacturers have chosen not to bundle.

\subsection{Visualized numerical examples}

Here, some numerical examples are visualized to provide abetter interpretation on how different scenarios behave. The sensitivity of numerical examples is set on price effect $(\beta)$ in order to also capture the change of optimal behavior due to its distance from degree of substitutability $(\gamma) \cdot \gamma$ is set constant, therefore, higher $\beta$ means the system is experiencing a relatively smaller $\gamma$. 
TABLE 13. Chain profit for different bundling strategies in different game structures for numerical examples (the MBRB strategy returned negative profit for these numerical examples).

\begin{tabular}{|c|c|c|c|c|c|c|c|c|c|c|c|c|c|}
\hline \multirow{2}{*}{$\begin{array}{l}\sum \pi \\
\text { Case }\end{array}$} & \multirow[b]{2}{*}{$\beta$} & \multicolumn{3}{|c|}{ MSB } & \multicolumn{3}{|c|}{ RSB } & \multicolumn{3}{|c|}{ MSSRS } & \multicolumn{3}{|c|}{ RSSMS } \\
\hline & & MNRN & MNRB & MBRB & MNRN & MNRB & MBRB & MNRN & MNRB & MBRB & MNRN & MNRB & MBRB \\
\hline \multirow{2}{*}{$\begin{array}{l}\text { (Symm, } \\
\alpha=3)\end{array}$} & 4.00 & 0.9 & 0.88 & 0.89 & 0.9 & 0.37 & 0.88 & 0.92 & 0.88 & 0.84 & 0.92 & 0.88 & 0.85 \\
\hline & 3.75 & 2.87 & 2.77 & 2.78 & 2.9 & 1.12 & 2.75 & 2.86 & 2.76 & 2.64 & 2.88 & 2.75 & 2.68 \\
\hline \multirow{2}{*}{$\begin{array}{l}\text { (Asym:1, } \\
\alpha=8)\end{array}$} & 3.25 & 212 & 208 & 207 & 207 & 84 & 199 & 214 & 209 & 205 & 211 & 205 & 202 \\
\hline & 3.01 & 926 & 936 & 935 & 715 & 2509 & 719 & 1136 & 1145 & 1135 & 924 & 932 & 925 \\
\hline \multirow{2}{*}{$\begin{array}{l}\text { (Asym:2, } \\
\alpha=1)\end{array}$} & 3.25 & 1.26 & 1.25 & 1.24 & 1.36 & 0.2 & 1.34 & 1.19 & 1.18 & 1.17 & 1.23 & 1.22 & 1.21 \\
\hline & 3.01 & 14.5 & 14.7 & 14.6 & 11.5 & 37.1 & 11.6 & 17.51 & 17.64 & 17.49 & 14.5 & 14.65 & 14.6 \\
\hline$\sum \pi$ & & \multicolumn{3}{|c|}{ MSB } & \multicolumn{3}{|c|}{ RSB } & \multicolumn{3}{|c|}{ MSSRS } & \multicolumn{3}{|c|}{ RSSMS } \\
\hline Case & $\beta$ & MNRN & MNRB & MBRB & MNRN & MNRB & MBRB & MNRN & MNRB & MBRB & MNRN & MNRB & MBRB \\
\hline \multirow{2}{*}{$\begin{array}{l}\text { (Symm, } \\
\alpha=3)\end{array}$} & 4.00 & 0.9 & 0.85 & 0.82 & 0.91 & 0.87 & - & 0.91 & 0.85 & 0.82 & 0.91 & 0.87 & 0.83 \\
\hline & 3.75 & 2.83 & 2.68 & 2.58 & 2.85 & 2.73 & - & 2.83 & 2.68 & 2.58 & 2.85 & 2.72 & 2.63 \\
\hline \multirow{2}{*}{$\begin{array}{l}\text { (Asym:1, } \\
\alpha=8)\end{array}$} & 3.25 & 217 & 211 & 207 & 216 & 210 & - & 215.6 & 209.9 & 205.8 & 212 & 206 & 202 \\
\hline & 3.01 & 1514 & 1519 & 1511 & 1332 & 1337 & - & 1422 & 1427 & 1419 & 1003 & 1010 & 1003.5 \\
\hline \multirow{2}{*}{$\begin{array}{l}\text { (Asym:2, } \\
\alpha=1)\end{array}$} & 3.25 & 1.14 & 1.12 & 1.11 & 1.16 & 1.15 & - & 1.07 & 1.06 & 1.05 & 1.11 & 1.10 & 1.09 \\
\hline & 3.01 & 22.9 & 23 & 22.85 & 20.3 & 20.4 & - & 21.54 & 21.62 & 21.50 & 15.5 & 15.7 & 15.6 \\
\hline
\end{tabular}

\subsubsection{Individual and chain profits}

Numerical results show that under symmetric settings, MNRN strategy always leads to highest chain profit and also market size (chain demand). The result holds for market size in both asymmetric cases, while in those cases there are values of $\beta$ for which the MNRB strategy leads to highest chain profit (Tab. 13).

The results in Table 13 reveal that chain profit tends to be lower for most of MNRN strategies in retailer leading games compared to manufacturer leading games. Lower $\beta$, meaning higher degree of substitution leads to higher chain profit. In asymmetric cases, MNRB is the superior strategy at the lower bound of $\beta$. In those cases even MBRB strategy outgoes the MNRN strategy. This holds for all game structures. Figure 11 shows a mesh diagram of colors for better understanding of how much profit each player gains from each game structure. In this figure, the profits have been normalized into the interval $[0,1]$; the white color representing 0 and black color representing 1 . Therefore the profits that are higher are darker.

Clearly the retailers experience more profit in retailer leading games and manufacturers in manufacturing leading games. In the symmetric case, manufacturer 2's profit tends to be higher than manufacturer 1's profit in MSSRS and MSSiii\&iv, while in MSSi\&ii manufacturer 1's profit is higher. Although in all these games manufacturer 1 is the first mover, in MSSRS and MSSiii, retailer 2 who belongs to manufacturer 1's channel has a simultaneous move (final move somehow) or a strict final move by which he maximizes his outcomes. While in MSSi, retailer 1 from manufacturer 1's channel is the one who makes the final move. The result also holds for the asymmetric case 1 , but not case 2 because of the high manufacturing costs manufacturer 2 incurs. Retailer 2's profit tends to be higher than retailer 1's profit in RSSMS and RSSiii\&iv for the same reason that retailer 2 is not the first mover in any of those structures.

\subsubsection{Individual demands, individual and bundle prices}

Although the ordinal relationships among profits do not tend to change much due to changes in degree of substitution, the demands, individual and bundle prices experience a lot of ordinal change. As an example, Figures 12 and 13 show the behavior of optimal demands and bundle prices, respectively for retailer leading Stackelberg games. The order of graph labels presented in the right side are only related to the upper bound of $\beta$, and it can be seen that they are different for the lower bound. 


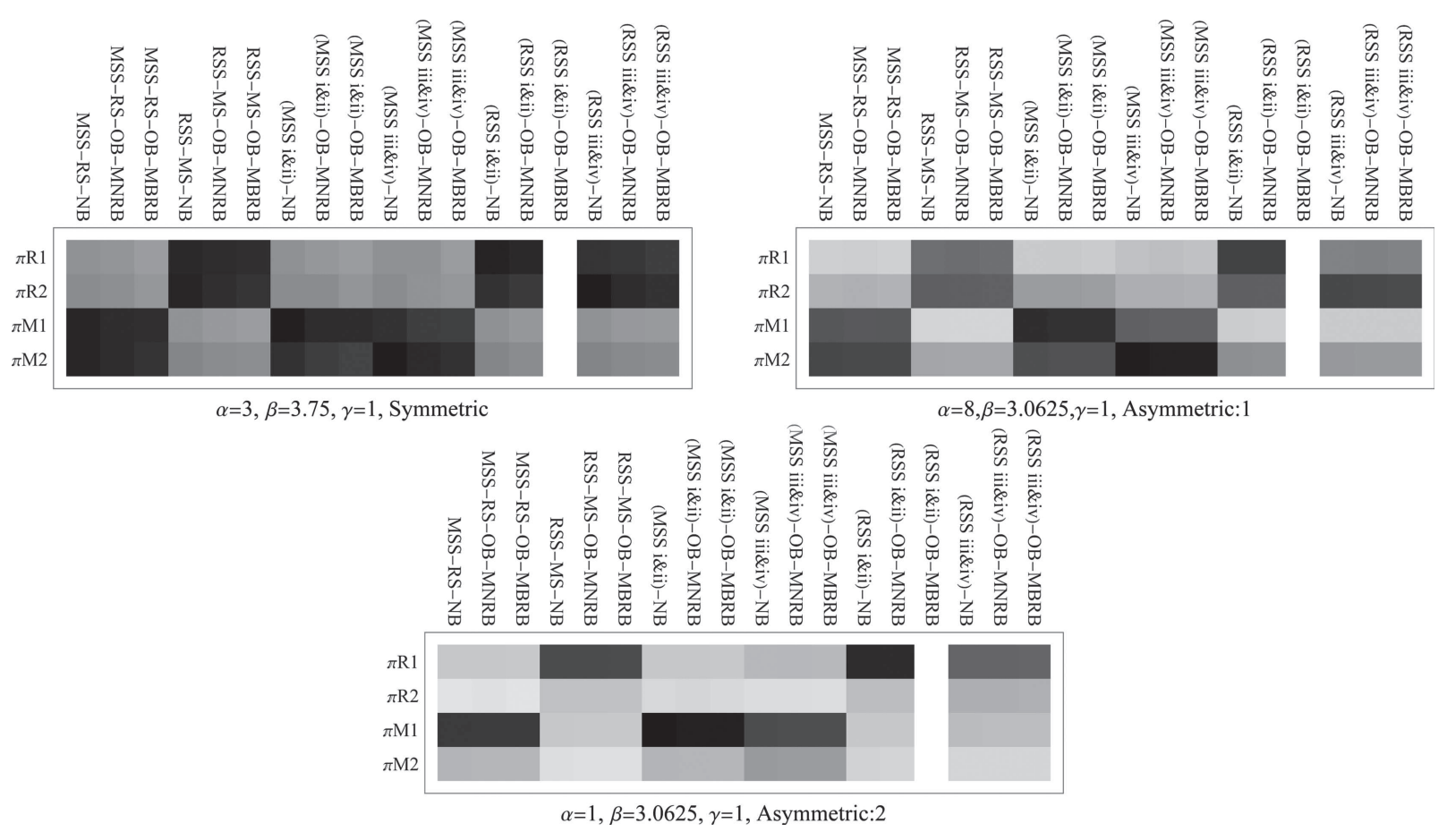

Figure 11. The profits of all players under different dame structures and bundling strategies for the numerical examples (the MBRB strategy returned negative profit for these numerical examples).

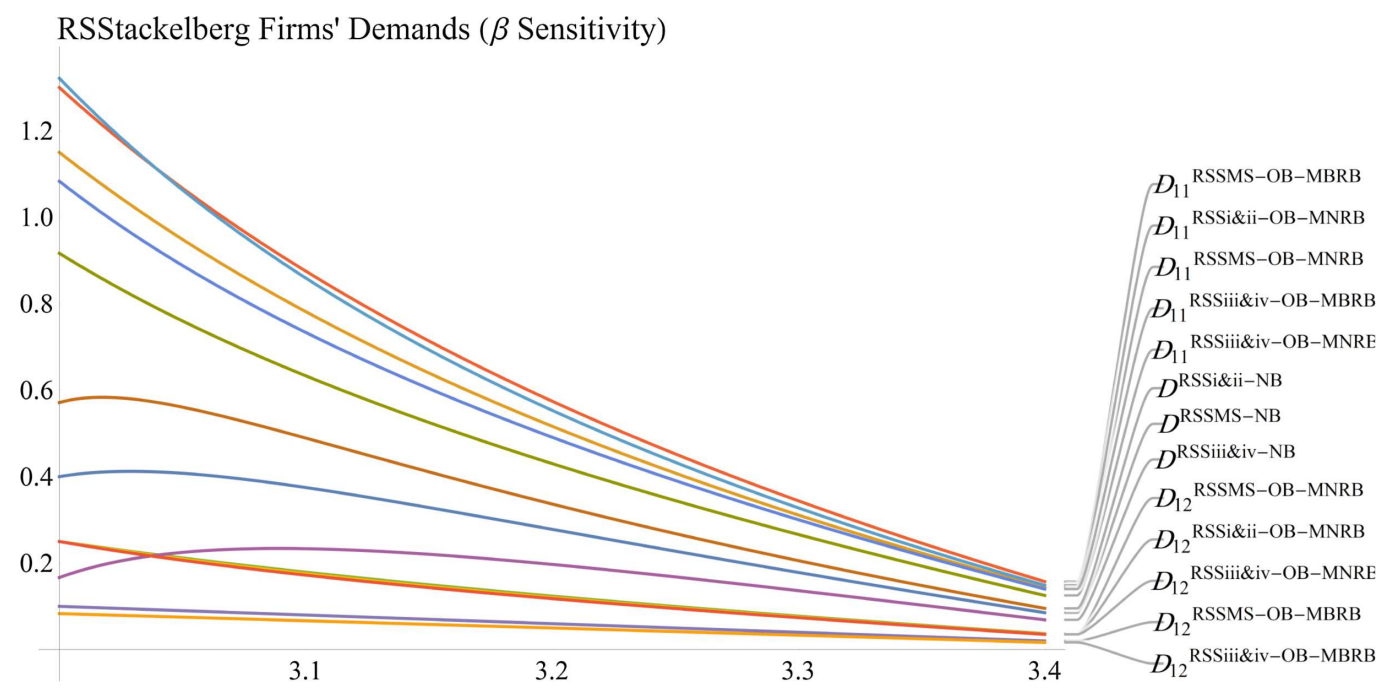

Figure 12. Retailer leading Stackelberg games' optimal demand for $\alpha=1, \beta \in(3,3.4), \lambda=1$, and asymmetric case 2 . 


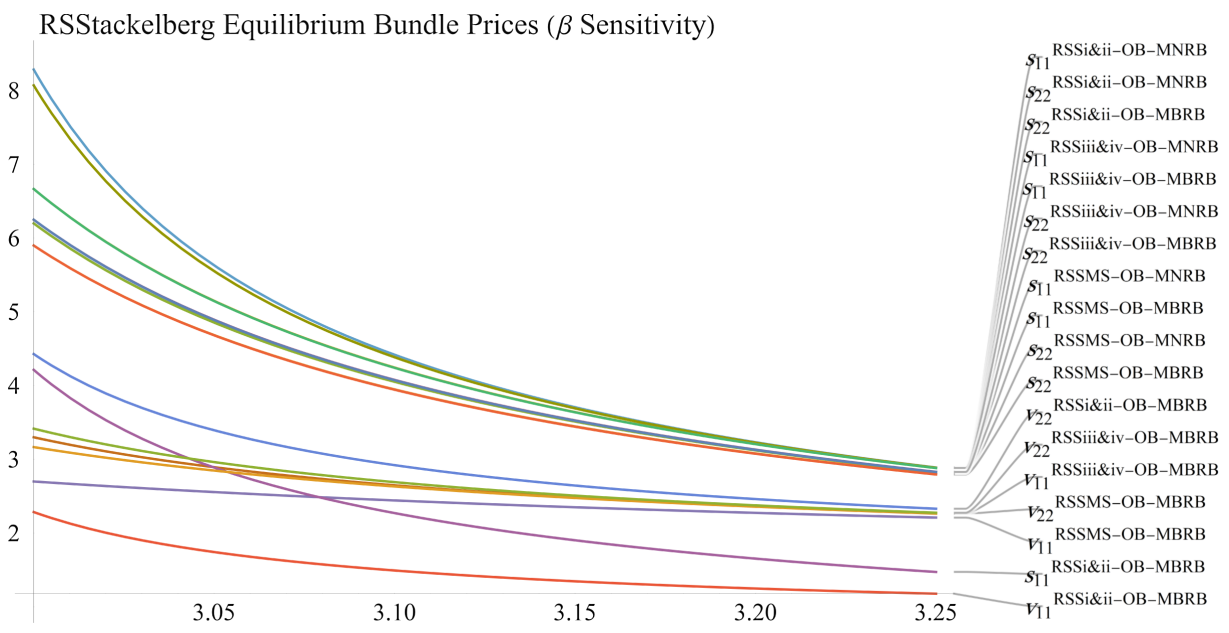

Figure 13. Retailer leading Stackelberg games' optimal bundle prices for $\alpha=1, \beta \in(3,3.25)$, $\lambda=1$, and asymmetric case 2 .

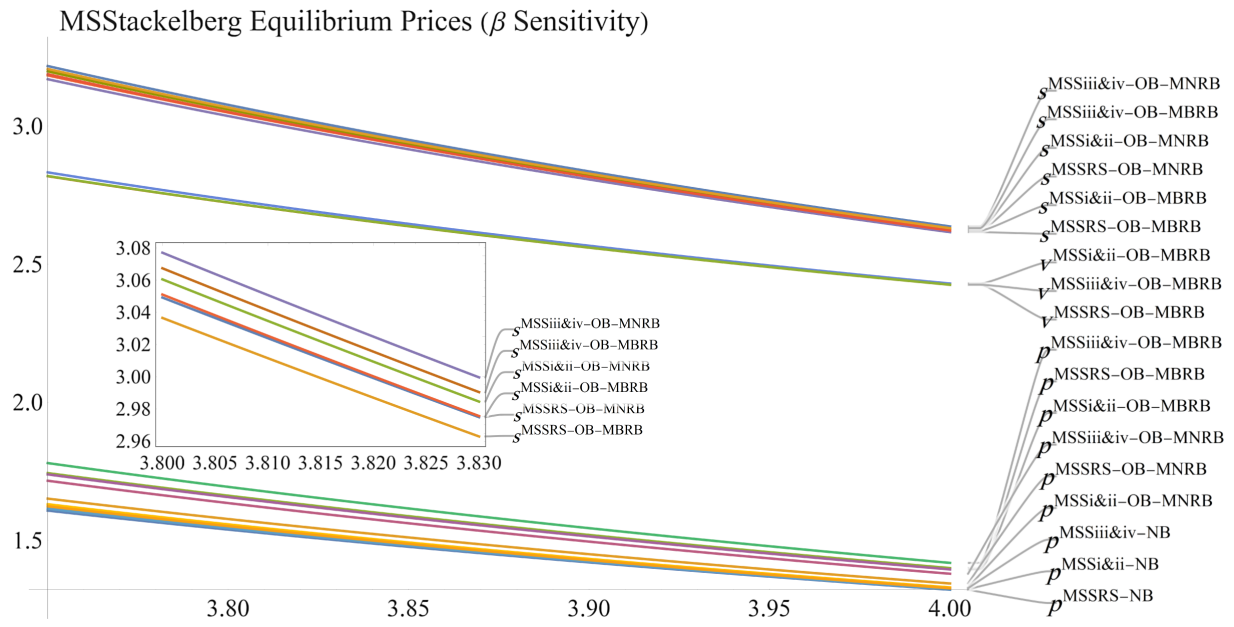

FiguRE 14. Manufacturer leading Stackelberg games' optimal individual and bundle retail prices for $\alpha=3, \beta \in(3.75,4), \lambda=1$, and symmetric case.

In the examples shown in Figure 12, we have set the upper bound of $\beta$, in a way that the final ordinal relations are revealed. Between $\beta$ values 3.2 and 3.4 the individual demands are struggling to find their final place which they eventually reach at the upper bound of 3.6. Interestingly, before the demand ordinal relations start changing, the bundle prices have found their places. This holds for the individual prices as well.

In symmetric settings, the ordinal relations of all variables do not tend to change, Figure 14. Also notice the difference between bundle wholesale prices and bundle retail prices which tends to remain approximately the same for all game structures. 


\section{Conclusions}

We analyzed pricing and bundling decisions from different aspects in a parallel-wise structured dual channel supply chain. The products were considered requisite complementary products leading to the final configuration of the market as two direct composites and two indirect composites. The key feature of our model was that the consumers have freedom of choice in buying each of the components of their required composite. For instance, a consumer can buy the hardware from one brand and the required software package from another brand. Considering the concept of market power and the possibility of combination of simultaneous and sequential moves in the game, we derived optimal retail and wholesale prices for two Bertrand game structures, and six Stackelberg game structures. Bundling strategies were used in both retailer-manufacturer and consumer-retailer relations. Our results showed interesting and reliable insights for managers who are seeking decision support for examining impacts of cost reduction scenarios and market power structures on optimal outcomes such as prices, demands and profits. The major findings are summarized as follows.

(1) Cost reduction with compensation did not change market size (sum of demands) but resulted in an increase (not necessarily equivalent) for all players in the supply network. In other words, the cake does not get bigger, just more delicious.

(2) Under cost asymmetry, coefficients of manufacturing costs played an important role in identifying the behavior of optimal price, demand and profit changes compared to each other. Especially, meaningful relations were observed among complementary and substitutable products forming the aspect of substitutable composites within a supply network with parallel supply channels.

(3) Cost reductions have various increasing and decreasing impacts in prices, demands and profits. Our results showed that the increasing effect on different products and players are different than the decreasing effect among different game structures. Meaningful relations are also derived for the comparison among complementary and substitutable products.

(4) Manufacturers incured different costs for providing their products. Our results showed that the differences between optimal retail and wholesale prices has a meaningful relation with the gaps between the manufacturing costs of complementary products. Interestingly, it was then concluded that it is in favor of manufacturers (profit-wise) when only the manufacturer with the higher gap between his/her complementary products' costs, practices cost reduction with compensation.

(5) The retail advantage granted to the retailer (the difference between one of the product's retail and wholesale prices, comparing to its complementary product), is much higher in retailer leading than manufacturer leading game structures. The retail advantage reached its highest level when in one of the fully sequential retailer leading games. While in manufacturer leading games, the highest level is granted when both retailers and manufacturer play Bertrand.

(6) Profit gains due to cost reduction were in favor of the players that had more market power. As the profit change moved along the ordinal pricing actions, it diminished based on meaningful relations with optimal price and demand changes which were derived carefully in terms of asymmetric cost coefficients.

(7) Comparing profit changes, showed that the fully sequential models are the last preferences in both retailer leading and manufacturer leading game structures. Both manufacturers and retailers prefered Bertrand (simultaneous) pricing games in order to achieve the highest profit changes due to possible cost cutting actions. This decision was also in benefit of the whole supply network's profit.

(8) Exact propositions showed that cost reduction led to no specific distinguish among bundling strategies. Because the optimal bundle prices (retail or wholesale) remained the same after applying cost reductions. The bundle prices acted as an intermediary in establishing the behavior of the main retail and wholesale prices within the supply network.

(9) Numerical insights showed that the game structure where manufacturers do not bundle and retailers bundle, is an unstable strategy for the whole supply network, because both layers (whether manufacturing or retailing) are reluctant to be the first movers no matter who holds more market power. Other than the exact insights which encourage simultaneous moves, these findings promote coordination among layers 
motivating them to either both bundle or not bundle the products in order to reach a more stable and profitable market.

In spite of linear demand functions, finite number of players and simple game structures, the optimal pricing solutions were very complex and seemed unbreakable. Many previous researches have tackled complexity by solving the models in symmetric settings and numerical examples. However, we showed that regardless of the mathematical sophistication that these structures can bring, it is still possible to derive managerial insights from masked parameters especially by applying comparative analysis on them. Therefore we encourage researchers to develop and generalize these models in many ways as follows:

(1) Under our assumptions, the methods did not succeed in finding a feasible solution for the case where manufacturers bundle and retailers do not bundle. The bundling strategy led to some conflicts concerning incoordination of demands. Researchers are also encouraged to further develop assumptions and practical methods for resolving conflicts arising from the inconsistency of the way suppliers sell to retailers and retailers sell to consumers. These methods are expected to fall in the category of dynamic inventory coordination and possible revenue sharing contracts.

(2) Marketing science can also be applied to the model in the context of consumer behavior. Considering bigger cross-price effect for cross-composites in the demand function of a same element composite and vice versa, means that they are more substitutable in the opinion of consumers; in other words the consumers appreciate diversity and are willing to choose a mixed composite instead of two products from the same brand. While lower cross-price effect can show more brand loyalty; the consumers are reluctant to buy a mixed composite and prefer a package of same brand products which is believed to have more compatibility.

(3) We calculated and discussed insights on profit changes due to the concurrent cost reduction with compensation strategy which can be considered as a coordination strategy as well. Due to the different profits for different game structures and bundling strategies, we did not proceed with further comparative analysis on the profits. One might be interested in applying consecutive concurrent cost reductions with compensation to observe the trajectory of profits; now made easier mathematically due to the results of this paper. It can also lead to insights on how the profits behave in response and whether the behavior will converge to a specific level.

(4) Eventually, we discussed various game structures with different set of rules. A very interesting problem arises when decision makers have desired results beforehand and need to make sure the game players behave in a way such that those outcomes are achieved. To do this, they should have the power to design the suitable game structure and rules. Instead of asking how the players would behave, one can control and lead their actions. This setting can be considered in the field of incentive compatibility and mechanism design. In order to achieve an optimal mechanism design, one should first evaluate the expected profit for different game scenarios. Then the problem would be turned into a maximzation of the expected profit subject to constraints of incentive compatibility and individual rationality. Allocation rules can vary based on the definition of cost reduction with compensation presented in this paper. However our study can also be rewritten in a way that covers the assumptions of mechanism design, but still it is worth noticing that the supply chain literature, specifically the ones discussing market powers and move sequences, lacks precise and focused studies concerning this field.

\section{Appendix A.}

\section{A.1. Proof of Proposition 4.1}

Here, we examine the Hessian matrix of retailers' profit functions for the case MSB-MNRN in Section 4.2, in order to verify their concavity. A same analysis can be performed for all the other game models. 
For the retailers, w.l.o.g, assume the case of retailer 1 . Because retailer 1 has only two control variables (responses), namely $p_{A 1}$ and $p_{B 1}$, its corresponding $2 \times 2$ Hessian matrix is as shown in equation (A.1).

$$
\text { Hess } \begin{aligned}
\pi_{R 1}\left(p_{A 1}, p_{B 1}\right) & =\left(\begin{array}{cc}
\frac{\partial^{2} \pi_{R 1}}{\partial p_{A 1}^{2}} & \frac{\partial \pi_{R 1}}{\partial p_{A 1} p_{B 1}} \\
\frac{\partial \pi_{R 1}}{\partial p_{B 1} p_{A 1}} & \frac{\partial^{2} \pi_{R 1}}{\partial p_{B 1}^{2}}
\end{array}\right) \\
& =\left(\begin{array}{cc}
-4(\beta-\gamma) & -2(\beta-3 \gamma) \\
-2(\beta-3 \gamma)-4(\beta-\gamma)
\end{array}\right) .
\end{aligned}
$$

In order to check whether the hessian matrix is indefinite, positive or negative definite, the first and second order leading principle minors of the matrix, should be checked. Let's name them $h_{1}$ and $h_{2}$, respectively as shown in equation (A.2). $h_{2}$ is simply the determinant of the whole Hessian matrix of equation (A.1).

$$
\begin{aligned}
h_{1} & =\left(\operatorname{Hess} \pi_{R 1}\right)_{11}=-4(\beta-\gamma), \\
h_{2} & =\left|\operatorname{Hess} \pi_{R 1}\right|=\left|\begin{array}{c}
-4(\beta-\gamma)-2(\beta-3 \gamma) \\
-2(\beta-3 \gamma)-4(\beta-\gamma)
\end{array}\right| \\
& =12 \beta^{2}-8 \beta \gamma-20 \gamma^{2} \\
& =4(\beta+\gamma)(3 \beta-5 \gamma) .
\end{aligned}
$$

Due to the assumption $\beta \geq 3 \gamma$, it can be verified that $h_{1}$ is negative and $h_{2}$ is positive, leading us to the result that Hess $\pi_{R 1}\left(p_{A 1}, p_{B 1}\right)$ is negative definite or $\pi_{R 1}\left(p_{A 1}, p_{B 1}\right)$ is a concave function. A same analysis can be performed for $\pi_{R 2}$. Therefore, it is concluded that the response retail prices acquired in equation (4.8) maximize their corresponding profit functions.

\section{A.2. Proof of Proposition 4.2}

Notice that Because the manufacturers are pricing leaders, due to the response maximization procedure depicted in equation (4.1), the retail prices are in fact functions of wholesale prices. Therefore, we should replace the optimal retail prices shown in equation (4.8) inside manufacturers' profit functions making them independent from any other control variable than the set of wholesale prices themselves. By doing so, we achieve manufacturer 1's profit function as simplified in equation (A.3). In fact, this is the profit function from which the stationary points in equation (4.10) are achieved.

$$
\begin{aligned}
\pi_{M 1}= & \frac{1}{3(7 \beta-17 \gamma)(5 \beta-3 \gamma)}\left(90 w_{A 1} \alpha \beta^{2}+90 w_{B 1} \alpha \beta^{2}-94 w_{A 1}^{2} \beta^{3}+4 w_{A 1} w_{A 2} \beta^{3}-118 w_{A 1} w_{B 1} \beta^{3}\right. \\
& -31 w_{A 2} w_{B 1} \beta^{3}-94 w_{B 1}^{2} \beta^{3}-31 w_{A 1} w_{B 2} \beta^{3}+4 w_{B 1} w_{B 2} \beta^{3}-204 w_{A 1} \alpha \beta \gamma \\
& -204 w_{B 1} \alpha \beta \gamma+370 w_{A 1}^{2} \beta^{2} \gamma+104 w_{A 1} w_{A 2} \beta^{2} \gamma+598 w_{A 1} w_{B 1} \beta^{2} \gamma \\
& +175 w_{A 2} w_{B 1} \beta^{2} \gamma+370 w_{B 1}^{2} \beta^{2} \gamma+175 w_{A 1} w_{B 2} \beta^{2} \gamma+104 w_{B 1} w_{B 2} \beta^{2} \gamma \\
& +90 w_{A 1} \alpha \gamma^{2}+90 w_{B 1} \alpha \gamma^{2}-322 w_{A 1}^{2} \beta \gamma^{2}-380 w_{A 1} w_{A 2} \beta \gamma^{2} \\
& -754 w_{A 1} w_{B 1} \beta \gamma^{2}-325 w_{A 2} w_{B 1} \beta \gamma^{2}-322 w_{B 1}^{2} \beta \gamma^{2}-325 w_{A 1} w_{B 2} \beta \gamma^{2} \\
& -380 w_{B 1} w_{B 2} \beta \gamma^{2}-18 w_{A 1}^{2} \gamma^{3}+288 w_{A 1} w_{A 2} \gamma^{3}+66 w_{A 1} w_{B 1} \gamma^{3} \\
& +237 w_{A 2} w_{B 1} \gamma^{3}-18 w_{B 1}^{2} \gamma^{3}+237 w_{A 1} w_{B 2} \gamma^{3}+288 w_{B 1} w_{B 2} \gamma^{3} \\
& +c_{B 1}\left(31 w_{A 2} \beta^{3}+94 w_{B 1} \beta^{3}-4 w_{B 2} \beta^{3}-175 w_{A 2} \beta^{2} \gamma-370 w_{B 1} \beta^{2} \gamma\right. \\
& -104 w_{B 2} \beta^{2} \gamma+325 w_{A 2} \beta \gamma^{2}+322 w_{B 1} \beta \gamma^{2}+380 w_{B 2} \beta \gamma^{2} \\
& -237 w_{A 2} \gamma^{3}+18 w_{B 1} \gamma^{3}-288 w_{B 2} \gamma^{3}-6 \alpha\left(15 \beta^{2}-34 \beta \gamma+15 \gamma^{2}\right) \\
& \left.+w_{A 1}\left(59 \beta^{3}-299 \beta^{2} \gamma+377 \beta \gamma^{2}-33 \gamma^{3}\right)\right) \\
& +c_{A 1}\left(-4 w_{A 2} \beta^{3}+59 w_{B 1} \beta^{3}+31 w_{B 2} \beta^{3}-104 w_{A 2} \beta^{2} \gamma-299 w_{B 1} \beta^{2} \gamma\right.
\end{aligned}
$$




$$
\begin{aligned}
& -175 w_{B 2} \beta^{2} \gamma+380 w_{A 2} \beta \gamma^{2}+377 w_{B 1} \beta \gamma^{2}+325 w_{B 2} \beta \gamma^{2} \\
& -288 w_{A 2} \gamma^{3}-33 w_{B 1} \gamma^{3}-237 w_{B 2} \gamma^{3}-6 \alpha\left(15 \beta^{2}-34 \beta \gamma+15 \gamma^{2}\right) \\
& \left.\left.+2 w_{A 1}\left(47 \beta^{3}-185 \beta^{2} \gamma+161 \beta \gamma^{2}+9 \gamma^{3}\right)\right)\right) .
\end{aligned}
$$

Similar to the retail profit function, the Hessian matrix of the function $\pi_{M 1}$ is shown in equation (A.4).

$$
\text { Hess } \begin{aligned}
\pi_{M 1}\left(w_{A 1}, w_{B 1}\right) & =\left(\begin{array}{cc}
\frac{\partial^{2} \pi_{M 1}}{\partial w_{A 1}^{2}} & \frac{\partial \pi_{M 1}}{\partial w_{A 1} w_{B 1}} \\
\frac{\partial \pi_{M 1}}{\partial w_{B 1} w_{A 1}} & \frac{\partial^{2} \pi_{M 1}^{2}}{\partial w_{B 1}^{2}}
\end{array}\right) \\
& =\left(\begin{array}{ll}
-\frac{4\left(47 \beta^{3}-185 \beta^{2} \gamma+161 \beta \gamma^{2}+9 \gamma^{3}\right)}{3(7 \beta-17 \gamma)(5 \beta-3 \gamma)} & -\frac{2(\beta-3 \gamma)\left(59 \beta^{2}-122 \beta \gamma+11 \gamma^{2}\right)}{3(7 \beta-17 \gamma)(5 \beta-3 \gamma)} \\
-\frac{2(\beta-3 \gamma)\left(59 \beta^{2}-122 \beta \gamma+11 \gamma^{2}\right)}{3(7 \beta-17 \gamma)(5 \beta-3 \gamma)} & -\frac{4\left(47 \beta^{3}-185 \beta^{2} \gamma+161 \beta \gamma^{2}+9 \gamma^{3}\right)}{3(7 \beta-17 \gamma)(5 \beta-3 \gamma)}
\end{array}\right) .
\end{aligned}
$$

Here, the determinants $h_{1}$ and $h_{2}$ are calculated as shown in equation (A.5).

$$
\begin{aligned}
& h_{1}=-\frac{4\left(47 \beta^{3}-185 \beta^{2} \gamma+161 \beta \gamma^{2}+9 \gamma^{3}\right)}{3(7 \beta-17 \gamma)(5 \beta-3 \gamma)}, \\
& h_{2}=\frac{4(3 \beta-5 \gamma)(\beta+\gamma)\left(17 \beta^{2}-46 \beta \gamma+\gamma^{2}\right)}{3(7 \beta-17 \gamma)(5 \beta-3 \gamma)} .
\end{aligned}
$$

The expressions $7 \beta-17 \gamma, 5 \beta-3 \gamma$ (in the denominators), and $3 \beta-5 \gamma$ are all positive due to the assumption $\beta \geq 3 \gamma$. Also, $\beta \geq 3 \gamma=\frac{51}{17} \gamma>\frac{46}{17} \gamma$ which leads to $17 \beta-46 \gamma>0$ concluding that $17 \beta^{2}-46 \beta \gamma+\gamma^{2}$ is also positive, therefore $h_{2}$ is positive. Also, the quadratic $47 \beta^{2}-185 \beta \gamma+161 \gamma^{2}$ is positive because $\beta^{2}$ 's coefficient is positive and $\Delta=\left(-\frac{181}{2}\right)^{2}-4 \times 47 \times 161<0$. Therefore, the expression $\beta\left(47 \beta^{2}-185 \beta \gamma+161 \gamma^{2}\right)+9 \gamma^{3}$ or $47 \beta^{3}-185 \beta^{2} \gamma+161 \beta \gamma^{2}+9 \gamma^{3}$ is positive, which leads to the conclusion that $h_{1}$ is negative. Hence, we conclude that Hess $\pi_{M 1}$ and Hess $\pi_{M 2}$ are also negative definite meaning that the wholesale price responses in equation (4.10) maximize their corresponding manufacturers' profits.

\section{A.3. Proof of Proposition 6.1}

Here we pursue with a mathematical experiment of how such a result is achieved for optimal prices and demands in the scenario MSB-MNRN. Consider the retail price solution for $p_{k i}$, as shown in equation (B.1). Let $C_{p_{k i}^{*}}$, be the part of optimal retail price $p_{k i}^{*}$, which are either independent of any manufacturing costs, or in terms of the expressions $\sum_{n \in\{A, B\}} \sum_{j \in\{1,2\}} c_{n j}$ (simply $c_{A 1}+c_{B 1}+c_{A 2}+c_{B 2}$ ), and $c_{k i}+c_{k, 3-i}-c_{\{A, B\} \backslash k, i}-c_{\{A, B\} \backslash k, 3-i}$ (e.g. for manufacturer 1, this expression represents $c_{A 1}+c_{B 1}-c_{A 2}-c_{B 2}$ ). We examine the situation w.l.o.g for product $A 1 . C_{p_{A 1}^{*}}$ is shown in equation (A.6). After subtracting $C_{p_{A 1}^{*}}$ from $p_{A 1}^{*}$, the leftovers would be as shown in equation (A.7).

$$
\begin{aligned}
C_{p_{A 1}^{*}} & =\frac{1}{62271}\left(\frac{1221\left(34 \alpha+4 \beta\left(c_{A 1}+c_{A 2}+c_{B 1}+c_{B 2}\right)\right)}{7 \beta-17 \gamma}\right. \\
& +\left(\frac{27676 \beta}{-5 \beta+3 \gamma}+\frac{7548 \beta(47 \beta-121 \gamma)}{31 \beta^{2}-66 \beta \gamma-33 \gamma^{2}}\right)\left(c_{A 1}-c_{A 2}+c_{B 1}-c_{B 2}\right) \\
& \left.+\frac{561\left(4 \beta(37 \beta-83 \gamma)\left(c_{A 1}+c_{A 2}+c_{B 1}+c_{B 2}\right)+74 \alpha(17 \beta-7 \gamma)\right)}{37 \beta^{2}-118 \beta \gamma+37 \gamma^{2}}\right) \\
p_{A 1}^{*}-C_{p_{A 1}^{*}}= & \frac{1}{62271}\left(16635 c_{A 1}+4\left(3687 c_{A 2}-4490 c_{B 1}-3232 c_{B 2}\right)\right) .
\end{aligned}
$$

Now due to the CRC (cost reduction with compensation) strategy, if manufacturer 1 decreases $c_{B 1}$ by $\delta$, $c_{A 1}$ correspondingly decreases by $\delta$. As shown in equation (A.8), the expressions $c_{A 1}+c_{B 1}+c_{A 2}+c_{B 2}$ and 
$c_{A 1}+c_{B 1}-c_{A 2}-c_{B 2}$ remain unchanged which justifies that $C_{p_{A 1}^{*}}$ also remains unchanged after applying CRC. The only part which is affected by the CRC, is equation (A.7).

$$
\begin{aligned}
& \left(c_{A 1}+\delta\right)+\left(c_{B 1}-\delta\right)+c_{A 2}+c_{B 2}=c_{A 1}+c_{B 1}+c_{A 2}+c_{B 2} \\
& \left(c_{A 1}+\delta\right)+\left(c_{B 1}-\delta\right)-c_{A 2}-c_{B 2}=c_{A 1}+c_{B 1}-c_{A 2}-c_{B 2} .
\end{aligned}
$$

Let $\left|p_{A 1}^{*}-C_{p_{A 1}^{*}}\right|_{\mathrm{CRC}(B 1)}$ represent the leftovers of $p_{A 1}^{*}$ after applying cost reduction with compensation in product $B 1$, see equation (A.9).

$$
\begin{aligned}
\left|p_{A 1}^{*}-C_{p_{A 1}^{*}}\right|_{\mathrm{CRC}(B 1)} & =\frac{1}{62271}\left(16635\left(c_{A 1}+\delta\right)+4\left(3687 c_{A 2}-4490\left(c_{B 1}-\delta\right)-3232 c_{B 2}\right)\right) \\
& =\left(p_{A 1}^{*}-C_{p_{A 1}^{*}}\right)+\frac{1}{62271}(16635+4 \times 4490) \delta, \\
& \Rightarrow \Delta_{p_{A 1}^{*}}^{\mathrm{CRC}(B 1)}=\frac{34595}{62271} \delta=\frac{5 \times 11 \times 17 \times 37}{3^{2} \times 11 \times 17 \times 37} \delta=\frac{5}{9} \delta .
\end{aligned}
$$

Therefore, cost reduction of manufacturing product $B 1$ by $\delta$ units, increases $p_{A 1}^{*}$ by $\frac{5}{9} \delta$. Now consider optimal wholesale price of scenario MSB-MNRN, see equation (4.10). $C_{w_{A 1}^{*}}$ is shown in equation (A.10). After subtracting $C_{w_{A 1}^{*}}$ from $w_{A 1}^{*}$, the leftovers would be as shown in equation (A.11).

$$
\begin{aligned}
C_{w_{A 1}^{*}}= & \frac{1}{1221}\left(\frac{148\left(c_{A 1}-c_{A 2}+c_{B 1}-c_{B 2}\right) \beta(37 \beta-99 \gamma)}{31 \beta^{2}-66 \beta \gamma-33 \gamma^{2}}\right. \\
& \left.+\frac{66\left(2\left(c_{A 1}+c_{A 2}+c_{B 1}+c_{B 2}\right) \beta(37 \beta-99 \gamma)+37 \alpha(5 \beta-3 \gamma)\right)}{37 \beta^{2}-118 \beta \gamma+37 \gamma^{2}}\right) \\
w_{A 1}^{*}-C_{w_{A 1}^{*}}= & \frac{1}{1221}\left(406 c_{A 1}+221 c_{A 2}-408 c_{B 1}-186 c_{B 2}\right) .
\end{aligned}
$$

After applying cost reduction with compensation in product $B 1$, the leftovers of $w_{A 1}^{*}$ will change as shown in equation (A.12).

$$
\begin{aligned}
\left|w_{A 1}^{*}-C_{w_{A 1}^{*}}\right|_{\mathrm{CRC}(B 1)} & =\frac{1}{1221}\left(406\left(c_{A 1}+\delta\right)+221 c_{A 2}-408\left(c_{B 1}-\delta\right)-186 c_{B 2}\right) \\
& =\left(w_{A 1}^{*}-C_{w_{A 1}^{*}}\right)+\frac{1}{1221}(406+408) \delta \\
& \Rightarrow \Delta_{w_{A 1}^{*}}^{\mathrm{CRC}(B 1)}=\frac{814}{1221} \delta=\frac{2 \times 11 \times 37}{3 \times 11 \times 37} \delta=\frac{2}{3} \delta
\end{aligned}
$$

Therefore, cost reduction of manufacturing product $B 1$ by $\delta$ units, increases $w_{A 1}^{*}$ by $\frac{2}{3} \delta$. Considering $\delta=1$, we have the couple $+\left(\frac{5}{9}, \frac{2}{3}\right)$ in first row and column of Table 3 . The same procedure can be proven for demand functions, and the other couples in the Tables 3 and 4 , are also derived with the same approach.

In summary, along with the defined notations, consider a same definition for notations $\omega_{k i}^{A 1}, \omega_{k i}^{B 1}, \omega_{k i}^{A 2}, \omega_{k i}^{B 2}$ for the coefficients of manufacturing costs in optimal wholesale prices. The optimal retail and wholesale prices are then written in the form shown in equation (A.13).

$$
\begin{aligned}
p_{k i}^{*}-C_{p_{k i}^{*}} & =\varphi_{k i}^{A 1} c_{A 1}+\varphi_{k i}^{B 1} c_{B 1}+\varphi_{k i}^{A 2} c_{A 2}+\varphi_{k i}^{B 2} c_{B 2}, \\
w_{k i}^{*}-C_{w_{k i}^{*}} & =\omega_{k i}^{A 1} c_{A 1}+\omega_{k i}^{B 1} c_{B 1}+\omega_{k i}^{A 2} c_{A 2}+\omega_{k i}^{B 2} c_{B 2} .
\end{aligned}
$$

In that case, when manufacturer 1 practices $\delta$ units of cost reduction for instance, for product $B 1$, the change in the first channel's optimal retail and wholesale prices are $\delta\left(\varphi_{k i}^{A 1}-\varphi_{k i}^{B 1}\right)$ and $\delta\left(\omega_{k i}^{A 1}-\omega_{k i}^{B 1}\right)$, respectively. The changes in demand are consequently derived based on the above expressions, and then profit changes are dervied based on both price and demand changes. For instance, consider $D_{12}$, see equation (4.2). 
After applying $\operatorname{CRC}(B 1)$, due to the calculated price changes in Table 3 , the change in $D_{12}$ is calculated as shown in equation (A.14). The other demand changes shown in Table 3 can be obtained through a same approach.

$$
\begin{aligned}
D_{12}= & \alpha-\beta\left(p_{A 1}+p_{B 2}\right)+\gamma\left(p_{A 1}+p_{B 1}\right)+\gamma\left(p_{A 2}+p_{B 2}\right)+\gamma\left(p_{A 2}+p_{B 1}\right) \\
\left.\stackrel{\operatorname{CRC}(B 1)}{\Rightarrow} D_{12}\right|_{\mathrm{CRC}(B 1)}= & \alpha-\beta\left(\left(p_{A 1}+\frac{5}{9} \delta\right)+\left(p_{B 2}-\frac{4}{9} \delta\right)\right) \\
& +\gamma\left(\left(p_{A 1}+\frac{5}{9} \delta\right)+\left(p_{B 1}-\frac{5}{9} \delta\right)\right)+\gamma\left(\left(p_{A 2}+\frac{4}{9} \delta\right)+\left(p_{B 1}-\frac{4}{9} \delta\right)\right) \\
& +\gamma\left(\left(p_{A 2}+\frac{4}{9} \delta\right)+\left(p_{B 1}-\frac{5}{9} \delta\right)\right) \\
= & D_{12}-\beta \delta\left(\frac{5}{9}-\frac{4}{9}\right)+\gamma \delta\left(\frac{4}{9}-\frac{5}{9}\right) \\
\Rightarrow & \left.D_{12}\right|_{\operatorname{CRC}(B 1)}-D_{12}=\Delta D_{12}=-\frac{1}{9} \delta(\beta+\gamma) .
\end{aligned}
$$

\section{A.4. Proof of Proposition 6.5}

We examine the case for optimal solution $p_{A 1}^{*}$ in game structure MSS-RS scenario MNRN, see equation (C.1). The coefficients of $c_{A 1}$ and $c_{B 1}$ in $p_{A 1}^{*}$ are as shown in equation (A.15).

$$
\begin{aligned}
& \varphi_{A 1}^{A 1}=\frac{862215}{249492}+\frac{2 \beta}{34(7 \beta-17 \gamma)}-\frac{\beta}{15 \beta-9 \gamma}+\frac{4 \beta(-13 \beta+35 \gamma)}{17 \beta^{2}-46 \beta \gamma+\gamma^{2}} \\
& \varphi_{A 1}^{B 1}=\frac{758260}{249492}+\frac{2 \beta}{34(7 \beta-17 \gamma)}-\frac{\beta}{15 \beta-9 \gamma}+\frac{4 \beta(-13 \beta+35 \gamma)}{17 \beta^{2}-46 \beta \gamma+\gamma^{2}} .
\end{aligned}
$$

We have $\psi^{1}\left|\varphi_{A 1}^{A 1}=\psi^{1}\right| \varphi_{A 1}^{B 1}=\frac{2 \beta}{34(7 \beta-17 \gamma)}-\frac{\beta}{15 \beta-9 \gamma}+\frac{4 \beta(-13 \beta+35 \gamma)}{17 \beta^{2}-46 \beta \gamma+\gamma^{2}}$ and $\psi^{\circ} \mid \varphi_{A 1}^{A 1}=\frac{862215}{249492} \cong 3.456 \neq 3.039 \cong$ $\frac{758260}{249492}=\psi^{\circ} \mid \varphi_{A 1}^{B 1}$. And that is how the numbers 3.456 and 3.039 in Table 6 are calculated; corresponding to columns $c_{A 1}$ and $c_{B 1}$, and row $p_{A 1}$, respectively.

The coefficients of $c_{A 2}$ and $c_{B 2}$ in $p_{A 1}^{*}$ are as shown in equation (A.16). It is clear that the dependent expression of cost coefficients $c_{A 2}$ and $c_{B 2}$ are different from their counterparts; $\psi^{1}\left|\varphi_{A 1}^{A 2}=\psi^{1}\right| \varphi_{A 1}^{B 2}$ and $\psi^{\circ} \mid \varphi_{A 1}^{A 2}=\frac{53151}{249492} \cong$ $0.213 \neq-0.370 \cong-\frac{92386}{249492}=\psi^{\circ} \mid \varphi_{A 1}^{B 2}$. And that is how the numbers 0.213 and -0.370 in Table 6 are calculated; corresponding to columns $c_{A 2}$ and $c_{B 2}$, and row $p_{A 1}$, respectively.

$$
\begin{aligned}
\varphi_{A 1}^{A 2}= & \frac{53151}{249492}+\frac{2 \beta}{34(7 \beta-17 \gamma)}+\frac{\beta}{15 \beta-9 \gamma} \\
& -\frac{4 \beta(37 \beta-99 \gamma)\left(139 \beta^{2}-1002 \beta \gamma+1227 \gamma^{2}\right)}{1223\left(569 \beta^{4}-2972 \beta^{3} \gamma+3414 \beta^{2} \gamma^{2}+1636 \beta \gamma^{3}-1223 \gamma^{4}\right)} \\
\varphi_{A 1}^{B 2}= & -\frac{92386}{249492}+\frac{2 \beta}{34(7 \beta-17 \gamma)}+\frac{\beta}{15 \beta-9 \gamma} \\
& -\frac{4 \beta(37 \beta-99 \gamma)\left(139 \beta^{2}-1002 \beta \gamma+1227 \gamma^{2}\right)}{1223\left(569 \beta^{4}-2972 \beta^{3} \gamma+3414 \beta^{2} \gamma^{2}+1636 \beta \gamma^{3}-1223 \gamma^{4}\right)} .
\end{aligned}
$$

The same results can be achieved by a similar attempt for all the other optimal prices, along with their cost coefficients.

\section{A.5. Proof of Proposition 6.9}

We examine the case for optimal price solutions of products $A 1$ and $B 1$ in game structure MSS-RS scenario MNRB, see equations (C.5) and (C.9). 
From Proposition 6.5 we know that $\psi^{1}\left|\varphi_{A 1}^{m j}=\psi^{1}\right| \varphi_{B 1}^{m j}$ is true $(\forall m \in\{A, B\})$. Therefore, we have:

$$
\begin{aligned}
p_{A 1}^{*}-p_{B 1}^{*} & =\sum_{j \in\{1,2\}} \sum_{m \in\{A, B\}}\left(\psi^{\circ}\left|\varphi_{A 1}^{m j}-\psi^{\circ}\right| \varphi_{B 1}^{m j}\right) c_{m j} \\
w_{A 1}^{*}-w_{B 1}^{*} & =\sum_{j \in\{1,2\}} \sum_{m \in\{A, B\}}\left(\psi^{\circ}\left|\omega_{A 1}^{m j}-\psi^{\circ}\right| \omega_{B 1}^{m j}\right) c_{m j} .
\end{aligned}
$$

The values in Table 6 are approximates of decimals which were calculated due to the fractions such as the $\psi^{\circ}$ part in equation (A.15). Here we use their exact fractions for an exact comprehension of the result in equation (A.17). Therefore, we have:

$$
\begin{aligned}
p_{A 1}^{*}-p_{B 1}^{*}= & \left(\frac{517314}{806820}-\frac{181139}{201705}\right) c_{A 1}+\left(\frac{181139}{806820}-\frac{517314}{806820}\right) c_{B 1} \\
& +\left(\frac{365109}{806820}-\left(-\frac{105536}{806820}\right)\right) c_{A 2}+\left(-\frac{105536}{806820}-\frac{365109}{806820}\right) c_{B 2} \\
= & \frac{1}{12}\left(5 c_{A 1}+7 c_{A 2}-5 c_{B 1}-7 c_{B 2}\right) \\
w_{A 1}^{*}-w_{B 1}^{*}= & \frac{1}{2}\left(c_{A 1}+c_{A 2}-c_{B 1}-c_{B 2}\right) .
\end{aligned}
$$

Finally, we have:

$$
\begin{aligned}
p_{A 1}^{*}-p_{B 1}^{*}-\left(w_{A 1}^{*}-w_{B 1}^{*}\right) & =\frac{1}{12}\left(5 c_{A 1}+7 c_{A 2}-5 c_{B 1}-7 c_{B 2}\right)-\frac{1}{2}\left(c_{A 1}+c_{A 2}-c_{B 1}-c_{B 2}\right) \\
& =\frac{1}{12}\left(-c_{A 1}+c_{B 1}+c_{A 2}-c_{B 2}\right) \\
& \Rightarrow p_{A 1}^{*}-w_{A 1}^{*}-\left(p_{B 1}^{*}-w_{B 1}^{*}\right)=\frac{1}{12}\left(c_{B 1}-c_{A 1}-\left(c_{B 2}-c_{A 2}\right)\right) .
\end{aligned}
$$

And that is how the number $\zeta=\frac{1}{12}$ corresponding to game structure MSSRS and channel 1 (CH1) in Table 7 is calculated. The same results can be achieved by a similar attempt for differences among all the other optimal retail and wholesale prices.

\section{A.6. Proof of Proposition 6.11}

The result achieved in equation (A.18) also justifies the forms discussed in Propsoition 6.11. Here we have:

$$
\begin{aligned}
p_{A 1}^{*}-p_{B 1}^{*} & =\overbrace{\frac{5}{12}}^{m_{p}}\left(c_{A 1}-c_{B 1}\right)+\overbrace{\frac{7}{12}}^{n_{p}}\left(c_{A 2}-c_{B 2}\right) \\
w_{A 1}^{*}-w_{B 1}^{*} & =\overbrace{\frac{1}{2}}^{m_{w}}\left(c_{A 1}-c_{B 1}\right)+\overbrace{\frac{1}{2}}^{n_{w}}\left(c_{A 2}-c_{B 2}\right) .
\end{aligned}
$$

The numbers $m_{p}$ and $m_{w}$ correspond to the values of column MSSRS for CH1 in Table 8. It is clear that $m_{p}+n_{p}=m_{w}+n_{w}=1$ and $m_{w}-m_{p}=n_{p}-n_{w}=\frac{1}{12}$ which is the value of $\zeta$ corresponding to MSSRS game structure for $\mathrm{CH} 1$, as shown in Table 7 . The same results can be achieved by a similar attempt for differences among all the other optimal retail and wholesale prices.

\section{A.7. Proof of Proposition 6.12}

Because, for each bundling strategy, the structure of profit functions are the same among game structures, we prove the result for $\operatorname{M1CRC}(B)$ and profits in bundling scenario MBRB, see equation (3.3). The same procedure can be applied for MNRN and MNRB scenarios. 
Let's investigate the case for $\pi_{M 1}$, w.l.o.g. Assume that $w_{A 1}^{*}$ increases by $x \delta$ units, after M1CRC is applied. Then, from Tables 3 and 4 we know that $w_{B 1}^{*}$ will decrease by $x \delta$ units. Also, if $D_{12}$ decreases by $y \delta$ units, $D_{21}$ increases by $y \delta$ units. For instance, for RSS-MS game structure $x=\frac{9}{10}$ and $y=\frac{1}{10}$. Therefore, we have:

$$
\begin{aligned}
\left.\pi_{M 1}\right|_{\mathrm{M} 1 \mathrm{CRC}}= & \left(\left(v_{11}-0\right)-\left(c_{A 1}+\delta\right)-\left(c_{B 1}-\delta\right)\right)\left(D_{11}-0\right) \\
& +\left(\left(w_{A 1}+x \delta\right)-\left(c_{A 1}+\delta\right)\right)\left(D_{12}-y \delta\right) \\
& +\left(\left(w_{B 1}-x \delta\right)-\left(c_{B 1}-\delta\right)\right)\left(D_{21}+y \delta\right) .
\end{aligned}
$$

By subtracting the $\pi_{M 1}$ before cost reduction, from $\left.\pi_{M 1}\right|_{\mathrm{M} 1 \mathrm{CRC}}$, the profit change is simplified as follows:

$$
\begin{aligned}
\Delta \pi_{M 1} & =\pi_{M 1}-\left.\pi_{M 1}\right|_{\mathrm{M} 1 \mathrm{CRC}} \\
& =\delta\left((1-x)\left(D_{21}-D_{12}\right)-x+y\left(c_{A 1}-c_{B 1}-\left(w_{A 1}-w_{B 1}\right)+2 \delta-2 x \delta\right)\right) .
\end{aligned}
$$

By replacing the demand functions from equation (3.2), the bundle prices are omitted and profit change is simplified as follows:

$$
\begin{aligned}
\Delta \pi_{M 1}= & c_{A 1} y \delta-\left(\left(p_{A 1}-p_{B 1}\right)-\left(p_{A 2}-p_{B 2}\right)\right)(-1+x)(\beta+\gamma) \\
& -y \delta\left(c_{B 1}+\left(w_{A 1}-w_{B 1}\right)+2(-1+x) \delta\right) .
\end{aligned}
$$

Recall that from equations (6.5) and (6.8) we have:

$$
\begin{aligned}
p_{A 1}-p_{B 1} & =m_{p}\left(c_{A 1}-c_{B 1}\right)+\left(1-m_{p}\right)\left(c_{A 2}-c_{B 2}\right) \\
p_{A 2}-p_{B 2} & =m_{p}\left(c_{A 2}-c_{B 2}\right)+\left(1-m_{p}\right)\left(c_{A 1}-c_{B 1}\right) \\
& \Rightarrow\left(p_{A 1}-p_{B 1}\right)-\left(p_{A 2}-p_{B 2}\right) \\
& =\left(2 m_{p}-1\right)\left(c_{A 1}-c_{B 1}\right)+\left(1-2 m_{p}\right)\left(c_{A 2}-c_{B 2}\right) .
\end{aligned}
$$

And from equation (6.6) we have:

$$
w_{A 1}-w_{B 1}=m_{w}\left(c_{A 1}-c_{B 1}\right)+\left(1-m_{w}\right)\left(c_{A 2}-c_{B 2}\right)
$$

where $m_{p}$ and $m_{w}$ are different for each game structure as shown in Table 8. Now by replacing equations (A.24) and (A.25) in equation (A.23), profit change is simplified as follow:

$$
\Delta \pi_{M 1}=\delta\left(y\left(1-m_{w}\right)+\left(2 m_{p}-1\right)(1-x)(\beta+\gamma)\right)\left(\left(c_{A 1}-c_{B 1}\right)-\left(c_{A 2}-c_{B 2}\right)\right)+2(1-x) y \delta^{2} .
$$

By investigating the numbers $x, y, m_{p}$, and $m_{w}$ in each game structure we can easily conclude that:

$$
y\left(1-m_{w}\right)=\left(2 m_{p}-1\right)(1-x)=(1-x) y .
$$

For instance, for RSB game structure we have $x=\frac{6}{7}, y=\frac{1}{7}, m_{p}=\frac{4}{7}$, and $m_{w}=\frac{6}{7}$. Therefore, we have $y\left(1-m_{w}\right)=\frac{1}{7} \times\left(1-\frac{1}{7}\right)=\frac{1}{49},\left(2 m_{p}-1\right)(1-x)=\left(2 \times \frac{4}{7}-1\right)\left(1-\frac{6}{7}\right)=\frac{1}{49}$ and $(1-x) y=\left(1-\frac{6}{7}\right) \times \frac{1}{7}=\frac{1}{49}$, which are all equivalent. The rational multiple mentioned in Proposition 6.12 , is in fact the above equivalent expressions. That is how the number $\frac{1}{49}$ is calculated corresponding to row $\Delta \pi_{M 1}$ and column RSB in Table 9 . Hence, the profit change of manufacturer 1 is as follows:

$$
\begin{aligned}
\Delta \pi_{M 1} & =\left(2 m_{p}-1\right)(1-x) \delta\left((1+\beta+\gamma)\left(\left(c_{A 1}-c_{B 1}\right)-\left(c_{A 2}-c_{B 2}\right)\right)+2 \delta\right) \\
& =\left(2 m_{p}-1\right)(1-x) \chi_{\mathrm{M} 1 \mathrm{CRC}(B)}(\delta) .
\end{aligned}
$$

Similar result can be verified for the retailers and manufacturer 2 . 


\section{Appendix B. Optimal retail prices of structures in Section 4; BERTRAND GAMES}

In Section 4 we showed some of the optimal prices in the form of best responses and in terms of optimal wholesale or retail prices. Here the exact equations of those prices are presented, which are in terms of costs and exogenous parameters.

\section{B.1. MSB-MNRN}

$$
\begin{aligned}
p_{k i}^{*}= & \frac{1}{62271}\left[16635 c_{k i}+4\left(3687 c_{k, 3-i}-4490 c_{\{A, B\} \backslash k, i}-3232 c_{\{A, B\} \backslash k, 3-i}\right)+\frac{1221\left(34 \alpha+4 \beta \sum_{\substack{n \in\{A, B\} \\
j \in\{1,2\}}} c_{n j}\right)}{7 \beta-17 \gamma}\right. \\
& +\left(\frac{27676 \beta}{-5 \beta+3 \gamma}+\frac{7548 \beta(47 \beta-121 \gamma)}{31 \beta^{2}-66 \beta \gamma-33 \gamma^{2}}\right)\left(c_{k, 1}+c_{k, 2}-c_{\{A, B\} \backslash k, 1}-c_{\{A, B\} \backslash k, 2}\right) \\
& \left.+\frac{561\left(4 \beta(37 \beta-83 \gamma) \sum_{\substack{n \in\{A, B\} \\
j \in\{1,2\}}} c_{n j}+74 \alpha(17 \beta-7 \gamma)\right)}{37 \beta^{2}-118 \beta \gamma+37 \gamma^{2}}\right]
\end{aligned}
$$

\section{B.2. MSB-MNRB}

$$
\begin{aligned}
p_{k i}^{*}= & \frac{1}{6390}\left[l-2\left(6618 c_{k i}-8292 c_{k, 3-i}+8393 c_{\{A, B\} \backslash k, i}-6872 c_{\{A, B\} \backslash k, 3-i}\right)+\frac{142\left(10 \alpha+\beta \sum_{\substack{n \in\{A, B\} \\
j \in\{1,2\}}} c_{n j}\right)}{2 \beta-5 \gamma}\right. \\
& +\left(\frac{1065 \beta\left(430 \beta^{2}-1963 \beta \gamma+2199 \gamma^{2}\right)}{28 \beta^{3}-126 \beta^{2} \gamma+135 \beta \gamma^{2}+9 \gamma^{3}}+\frac{710 \beta}{-2 \beta+3 \gamma}\right)\left(c_{k, 1}+c_{k, 2}-c_{\{A, B\} \backslash k, 1}-c_{\{A, B\} \backslash k, 2}\right) \\
& \left.+710 \alpha\left(34 \beta^{2}-103 \beta \gamma+73 \gamma^{2}\right)+5 \beta\left(214 \beta^{2}-775 \beta \gamma+691 \gamma^{2}\right) \sum_{n \in\{A, B\}} c_{n j}\right] \\
& \left.+\frac{12 \beta^{3}-70 \beta^{2} \gamma+127 \beta \gamma^{2}-71 \gamma^{3}}{s_{i i}^{*}=}\right], \\
& +\alpha(2 \beta-3 \gamma)\left(56 \beta^{3}-316 \beta^{2} \gamma+567 \beta \gamma^{2}-321 \gamma^{3}\right)\left(28 \beta^{3}-126 \beta^{2} \gamma+135 \beta \gamma^{2}+9 \gamma^{3}\right) \\
& +12\left(-39 c_{A, i}+11 c_{A, 3-i}-39 c_{B, i}+11 c_{B, 3-i}\right) \beta^{3} \gamma+6\left(205 c_{A, i}-97 c_{A, 3-i}+205 c_{B, i}-97 c_{B, 3-i}\right) \beta^{2} \gamma^{2} \\
& +9\left(-151 c_{A, i}+108 c_{A, 3-i}-151 c_{B, i}+108 c_{B, 3-i}\right) \beta \gamma^{3} \\
& \left.\left.+3\left(173 c_{A, i}-182 c_{A, 3-i}+173 c_{B, i}-182 c_{B, 3-i}\right) \gamma^{4}\right]\right] \\
& /\left(3(2 \beta-5 \gamma)(2 \beta-3 \gamma)\left(12 \beta^{3}-70 \beta^{2} \gamma+127 \beta \gamma^{2}-71 \gamma^{3}\right)\left(28 \beta^{3}-126 \beta^{2} \gamma+135 \beta \gamma^{2}+9 \gamma^{3}\right)\right) .
\end{aligned}
$$




\section{B.3. MSB-MBRB}

$$
\begin{aligned}
p_{k i}^{*}= & \frac{1}{102240}\left[-105951 c_{k i}+158169 c_{k, 3-i}-162751 c_{\{A, B\} \backslash k, i}+112729 c_{\{A, B\} \backslash k, 3-i}\right. \\
& -\left(\frac{4260 \beta(4 \beta-11 \gamma)}{4 \beta^{2}-15 \beta \gamma+12 \gamma^{2}}+\frac{11360 \beta}{-2 \beta+3 \gamma}\right)\left(c_{k, 1}+c_{k, 2}-c_{\{A, B\} \backslash k, 1}-c_{\{A, B\} \backslash k, 2}\right) \\
& \left.+\frac{355\left(32 \alpha(8 \beta-11 \gamma)+\beta(4 \beta-\gamma) \sum_{\substack{n \in\{A, B\} \\
j \in\{1,2\}}} c_{n j}\right)}{4 \beta^{2}-17 \beta \gamma+16 \gamma^{2}}+\frac{2272\left(10 \alpha+\beta \sum_{\substack{n \in\{A, B\} \\
j \in\{1,2\}}} c_{n j}\right)}{2 \beta-5 \gamma}\right) \\
& +\frac{17040 \beta\left(262 \beta^{2}-1197 \beta \gamma+1341 \gamma^{2}\right)}{28 \beta^{3}-126 \beta^{2} \gamma+135 \beta \gamma^{2}+9 \gamma^{3}}\left(c_{k, 1}+c_{k, 2}-c_{\{A, B\} \backslash k, 1}-c_{\{A, B\} \backslash k, 2}\right) \\
& \quad 80\left(142 \alpha(5 \beta-9 \gamma)(2 \beta-3 \gamma)+\beta\left(262 \beta^{2}-1197 \beta \gamma+1341 \gamma^{2}\right) \sum_{n \in\{A, B\}} c_{n j}\right) \\
& \left.+\frac{12 \beta^{3}-70 \beta^{2} \gamma+127 \beta \gamma^{2}-71 \gamma^{3}}{s_{i i}}\right] \\
& {\left[2 \alpha(2 \beta-3 \gamma)\left(3 \beta^{2}-12 \beta \gamma+11 \gamma^{2}\right)\left(4 \beta^{2}-15 \beta \gamma+12 \gamma^{2}\right)\right.} \\
& +(\beta-2 \gamma)\left(2 \beta^{2}-8 \beta \gamma+7 \gamma^{2}\right)\left[8\left(c_{A, i}+c_{B, i}\right) \beta^{3}+6\left(-8 c_{A, i}+c_{A, 3-i}-8 c_{B, i}+c_{B, 3-i}\right) \beta^{2} \gamma\right. \\
& \left.\left.+3\left(31 c_{A, i}-8 c_{A, 3-i}+31 c_{B, i}-8 c_{B, 3-i}\right) \beta \gamma^{2}+2\left(-29 c_{A, i}+11 c_{A, 3-i}-29 c_{B, i}+11 c_{B, 3-i}\right) \gamma^{3}\right]\right] \\
& /\left[(2 \beta-5 \gamma)(2 \beta-3 \gamma)\left(4 \beta^{2}-15 \beta \gamma+12 \gamma^{2}\right)\left(4 \beta^{2}-17 \beta \gamma+16 \gamma^{2}\right)\right] .
\end{aligned}
$$

\section{B.4. RSB-MNRN}

The best response wholesale prices are calculated as follows:

$$
w_{k i}^{*}=\frac{2 \alpha}{3 \beta-5 \gamma}+c_{k i}-p_{k i}+\frac{\beta+\gamma}{3 \beta-5 \gamma} p_{k, 3-i}-\frac{2 \beta-6 \gamma}{3 \beta-5 \gamma} p_{\{A, B\} \backslash k, 3-i} .
$$

The best responses of wholesale prices in this case are independent of the mutual product type's price from the same manufacturer. The optimal retail prices are then calculated as follows:

$$
\begin{aligned}
p_{k i}^{*}= & \frac{1}{287}\left(-268 c_{k i}+429 c_{k, 3-i}-432 c_{\{A, B\} \backslash k, i}+306 c_{\{A, B\} \backslash k, 3-i}+\frac{1722 \alpha+84 \beta \sum_{\substack{n \in\{A, B\} \\
j \in\{1,2\}}} c_{n j}}{15 \beta-41 \gamma}\right. \\
& \left.+\frac{3444\left(c_{k i}-c_{k, 3-i}+c_{\{A, B\} \backslash k, i}-c_{\{A, B\} \backslash k, 3-i}\right) \beta}{9 \beta+\gamma}\right) .
\end{aligned}
$$

We have separated the common statement between the optimal retail prices in which there is an expression including sum of the unit manufacturing costs. All other expressions switch coefficients based on the product 
type and the firm selling it. The final exact form of the optimal wholesale price is as follows.

$$
\begin{aligned}
w_{k i}^{*}= & \frac{1}{119}\left(482 c_{k i}-419 c_{k, 3-i}+414 c_{\{A, B\} \backslash k, i}-470 c_{\{A, B\} \backslash k, 3-i}\right) \\
& +\frac{68 \alpha+18 \beta \sum_{\substack{n \in\{A, B\} \\
j \in\{1,2\}}} c_{n j}}{153(26 \beta-51 \gamma)}+\frac{4 \alpha}{9(\beta-3 \gamma)} \\
& -\frac{2 \beta(11 \beta-23 \gamma)}{6 \beta^{2}-13 \beta \gamma+\gamma^{2}}\left(c_{k, 1}+c_{k, 2}-c_{\{A, B\} \backslash k, 1}-c_{\{A, B\} \backslash k, 2}\right) .
\end{aligned}
$$

\section{B.5. RSB-MNRB}

The optimal wholesale and retail prices of RSB-MNRB game structure are given as follows:

$$
\begin{aligned}
p_{k i}^{*}= & \frac{1}{119}\left(482 c_{k i}-419 c_{k, 3-i}+414 c_{\{A, B\} \backslash k, i}-470 c_{\{A, B\} \backslash k, 3-i}\right) \\
& +\frac{68 \alpha+18 \beta \sum_{\substack{n \in\{A, B\} \\
j \in\{1,2\}}} c_{n j}}{153(26 \beta-51 \gamma)}+\frac{4 \alpha}{9(\beta-3 \gamma)}-\frac{2\left(c_{k i}-c_{k, 3-i}+c_{\{A, B\} \backslash k, i}-c_{\{A, B\} \backslash k, 3-i}\right) \beta(11 \beta-23 \gamma)}{6 \beta^{2}-13 \beta \gamma+\gamma^{2}}, \\
s_{i i}^{*}= & {\left[\left(\alpha(23 \beta-45 \gamma)\left(6 \beta^{2}-13 \beta \gamma+\gamma^{2}\right)+(\beta-3 \gamma)(\beta-2 \gamma)\left(2\left(11 c_{A i}-2 c_{A, 3-i}+11 c_{B i}-2 c_{B, 3-i}\right) \beta^{2}\right.\right.\right.} \\
& \left.\left.\left.+3\left(-28 c_{A i}+15 c_{A, 3-i}-28 c_{B i}+15 c_{B, 3-i}\right) \beta \gamma+3\left(26 c_{A i}-25 c_{A, 3-i}+26 c_{B i}-25 c_{B, 3-i}\right) \gamma^{2}\right)\right)\right] \\
& /\left[(26 \beta-51 \gamma)(\beta-3 \gamma)\left(6 \beta^{2}-13 \beta \gamma+\gamma^{2}\right)\right], \\
w_{k i}^{*}= & \frac{1}{119}\left(219 c_{k i}-121 c_{k, 3-i}+117 c_{\{A, B\} \backslash k, i}-138 c_{\{A, B\} \backslash k, 3-i}\right) \\
& -\frac{238 \alpha+63 \beta \sum_{\substack{n \in\{A, B\} \\
j \in\{1,2\}}} c_{n j}}{306(26 \beta-51 \gamma)}+\frac{2 \alpha}{9(\beta-3 \gamma)}+\frac{\left(c_{k i}-c_{k, 3-i}+c_{\{A, B\} \backslash k, i}-c_{\{A, B\} \backslash k, 3-i i}\right) \beta(-13 \beta+27 \gamma)}{2\left(6 \beta^{2}-13 \beta \gamma+\gamma^{2}\right)} .
\end{aligned}
$$

Like the RSB-MNRN model, the sum of all unit manufacturing costs appears as a common statement in both retail and wholesale prices, however, not in the optimal bundle prices. The same occurrence can also be seen in the solutions of RSB-MBRB game.

\section{B.6. RSB-MBRB}

The optimal retail and wholesale prices for the MBRB case are obtained as follows:

$$
\begin{aligned}
& p_{k i}^{*}=\frac{1}{385}\left(\begin{array}{c}
328 c_{k i}-123 c_{k, 3-i}+108 c_{\{A, B\} \backslash k, i}-288 c_{\{A, B\} \backslash k, 3-i} \\
660 \alpha+30 \beta \sum_{\substack{n \in\{A, B\} \\
j \in\{1,2\}}} c_{n j} \\
+\frac{4 \beta-11 \gamma}{4 \beta-\left(c_{k i}-c_{k, 3-i}+c_{\{A, B\} \backslash k, i}-c_{\{A, B\} \backslash k, 3-i}\right) \beta}
\end{array}\right) \text {, } \\
& s_{i i}^{*}=\frac{3 \alpha(4 \beta-5 \gamma)+(\beta-2 \gamma)\left(4\left(c_{k i}+c_{\{A, B\} \backslash k, i}\right) \beta+\left(-8 c_{k i}+3 c_{k, 3-i}-8 c_{\{A, B\} \backslash k, i}+3 c_{\{A, B\} \backslash k, 3-i}\right) \gamma\right)}{(4 \beta-11 \gamma)(4 \beta-5 \gamma)}, \\
& w_{k i}^{*}=\frac{1}{385}\left(\begin{array}{c}
366 c_{k i}-41 c_{k, 3-i}+36 c_{\{A, B\} \backslash k, i}-96 c_{\{A, B\} \backslash k, 3-i} \\
220 \alpha+10 \beta \sum_{\substack{n \in\{A, B\} \\
j \in\{1,2\}}} c_{n j} \\
+\frac{4 \beta-11 \gamma}{+}-\frac{154\left(c_{k i}-c_{k, 3-i}+c_{\{A, B\} \backslash k, i}-c_{\{A, B\} \backslash k, 3-i}\right) \beta}{4 \beta-5 \gamma}
\end{array}\right), \\
& v_{i i}^{*}=\frac{4 \beta\left(\alpha+3\left(c_{A i}+c_{B i}\right) \beta\right)+\left(-5 \alpha+\left(-48 c_{A i}+c_{A, 3-i}-48 c_{B i}+c_{B, 3-i}\right) \beta\right) \gamma}{(4 \beta-11 \gamma)(4 \beta-5 \gamma)} \\
& +\frac{2\left(21 c_{A i}-c_{A, 3-i}+21 c_{B i}-c_{B, 3-i}\right) \gamma^{2}}{(4 \beta-11 \gamma)(4 \beta-5 \gamma)} .
\end{aligned}
$$




\section{Appendix C. Optimal prices of game structures in Section 5; STACKELBERG GAMES}

The solutions of each Stackelberg game structure are congested mathematically and would consume a lot of pages. However, for the readers to capture some elaboration on exact optimal prices, we have provided the solutions of MSS-RS games which also contain intra-layer sequential moves. The rest of the models' exact solutions can also be provided due to request. For the elaboration to help understand the insights of this paper better, it is suggested for the readers to look for expressions that include unit manufacturing costs especially the ones that have all four of the manufacturing costs within them. Although complicated, the parallel structure of the supply chain has led to a combination for coefficients of most of these expressions in a way that for symmetric costs these expressions will equal to zero. Another interesting fact about the optimal prices in Stackelberg games is that no matter how the structure is, the prices of direct complementary products are mutually symmetric although different with the other channel's optimal structures.

\section{C.1. MSS-RS-MNRN}

In the MSS-RS-MNRN, where manufacturers are leading and playing Stackelberg while retailers play simultaneously and none of them choose to bundle, the optimal retail and wholesale prices are derived as follows. Notice that the expressions in optimal prices have a symmetric structure for each channel but are differently structured among them. Also, the wholesale prices of manufacturer 1 (the manufacturer who moves first in the Stackelberg) is independent of its complementary product's manufacturing cost. This explains the corresponding zeros in Table 6.

$$
\begin{aligned}
p_{k, 1}^{*}= & \frac{862215 c_{k, 1}+53151 c_{k, 2}+758260 c_{\{A, B\} \backslash k, 1}-92386 c_{\{A, B\} \backslash k, 2}}{249492}+\frac{17 \alpha+2 \beta \sum_{\substack{n \in\{A, B\} \\
j \in\{1,2\}}} c_{n j}}{34(7 \beta-17 \gamma)} \\
& -\frac{\left(c_{k, 1}-c_{k, 2}+c_{\{A, B\} \backslash k, 1}-c_{\{A, B\} \backslash k, 2}\right) \beta}{15 \beta-9 \gamma}+\frac{\alpha(\beta+\gamma)+4\left(c_{k, 1}+c_{\{A, B\} \backslash k, 1}\right) \beta(-13 \beta+35 \gamma)}{17 \beta^{2}-46 \beta \gamma+\gamma^{2}} \\
& +\frac{1223 \alpha(5 \beta-3 \gamma)\left(31 \beta^{2}-66 \beta \gamma-33 \gamma^{2}\right)-4\left(c_{k, 2}+c_{\{A, B\} \backslash k, 2}\right) \beta(37 \beta-99 \gamma)\left(139 \beta^{2}-1002 \beta \gamma+1227 \gamma^{2}\right)}{1223\left(569 \beta^{4}-2972 \beta^{3} \gamma+3414 \beta^{2} \gamma^{2}+1636 \beta \gamma^{3}-1223 \gamma^{4}\right)},
\end{aligned}
$$

$$
\begin{aligned}
l p_{k, 2}^{*}= & \frac{-267837 c_{k, 1}+28116 c_{k, 2}-288628 c_{\{A, B\} \backslash k, 1}-13466 c_{\{A, B\} \backslash k, 2}}{62373}+\frac{17 \alpha+2 \beta \sum_{\substack{n \in\{A, B\} \\
j \in\{1,2\}}} c_{n j}}{34(7 \beta-17 \gamma)} \\
& -\frac{\left(c_{k, 2}-c_{k, 1}+c_{\{A, B\} \backslash k, 2}-c_{\{A, B\} \backslash k, 1}\right) \beta}{15 \beta-9 \gamma}+\frac{4\left(c_{k, 1}+c_{\{A, B\} \backslash k, 1}\right) \beta(37 \beta-99 \gamma)+\alpha(5 \beta-3 \gamma)}{2\left(17 \beta^{2}-46 \beta \gamma+\gamma^{2}\right)} \\
& +\frac{9784 \alpha(\beta+\gamma)\left(13 \beta^{2}-38 \beta \gamma+13 \gamma^{2}\right)+32\left(c_{k, 2}+c_{\{A, B\} \backslash k, 2}\right) \beta(13 \beta-35 \gamma)\left(121 \beta^{2}-398 \beta \gamma+313 \gamma^{2}\right)}{1223\left(569 \beta^{4}-2972 \beta^{3} \gamma+3414 \beta^{2} \gamma^{2}+1636 \beta \gamma^{3}-1223 \gamma^{4}\right)},
\end{aligned}
$$

$$
\begin{aligned}
w_{k, 1}^{*}= & {\left[\beta^{3}\left(310 \alpha+\left(569 c_{k, 1}+259 c_{k, 2}-310 c_{\{A, B\} \backslash k, 2}\right) \beta\right)\right.} \\
& -2 \beta^{2}\left(423 \alpha+2\left(743 c_{k, 1}+299 c_{k, 2}-444 c_{\{A, B\} \backslash k, 2}\right) \beta\right) \gamma \\
& +6 \beta\left(11 \alpha+\left(569 c_{k, 1}+135 c_{k, 2}-434 c_{\{A, B\} \backslash k, 2}\right) \beta\right) \gamma^{2}+2\left(99 \alpha+818\left(c_{k, 1}+c_{k, 2}\right) \beta\right) \gamma^{3} \\
& \left.+\left(-1223 c_{k, 1}-629 c_{k, 2}+594 c_{\{A, B\} \backslash k, 2}\right) \gamma^{4}\right] /\left(1138 \beta^{4}-5944 \beta^{3} \gamma+6828 \beta^{2} \gamma^{2}+3272 \beta \gamma^{3}-2446 \gamma^{4}\right),
\end{aligned}
$$


S1700

S. KEYHANIAN $E T A L$.

$$
\begin{aligned}
w_{k, 2}^{*}= & \frac{1}{4892}\left[-20791 c_{k, 1}+2445 c_{k, 2}-22014 c_{\{A, B\} \backslash k, 1}-1224 c_{\{A, B\} \backslash k, 2}\right. \\
& +\frac{2446\left(4\left(c_{k, 1}+c_{\{A, B\} \backslash k, 1}\right) \beta(37 \beta-99 \gamma)+\alpha(5 \beta-3 \gamma)\right)}{17 \beta^{2}-46 \beta \gamma+\gamma^{2}} \\
& \left.+\frac{32\left(c_{k, 2}+c_{\{A, B\} \backslash k, 2}\right) \beta(37 \beta-99 \gamma)(13 \beta-35 \gamma)(23 \beta-\gamma)+9784 \alpha(7 \beta-17 \gamma)(5 \beta-3 \gamma)(\beta+\gamma)}{569 \beta^{4}-2972 \beta^{3} \gamma+3414 \beta^{2} \gamma^{2}+1636 \beta \gamma^{3}-1223 \gamma^{4}}\right] .
\end{aligned}
$$

\section{C.2. MSS-RS-MNRB}

$$
\begin{aligned}
& p_{k, 1}^{*}=\left[\frac{517314 c_{k, 1}+365109 c_{k, 2}+181139 c_{\{A, B\} \backslash k, 1}-105536 c_{\{A, B\} \backslash k, 2}}{806820}\right. \\
& +\frac{\beta}{24 \beta-36 \gamma}\left(c_{k, 1}-c_{k, 2}+c_{\{A, B\} \backslash k, 1}-c_{\{A, B\} \backslash k, 2}\right)+\frac{10 \alpha+\beta}{60(2 \beta-5 \gamma)} \sum_{\substack{n \in\{A, B\} \\
j \in\{1,2\}}} c_{n j} \\
& +\frac{\left(c_{k, 1}+c_{\{A, B\} \backslash k, 1}\right) \beta\left(-242 \beta^{2}+1143 \beta \gamma-1303 \gamma^{2}\right)+17 \alpha\left(2 \beta^{2}+3 \beta \gamma-13 \gamma^{2}\right)}{68\left(16 \beta^{3}-84 \beta^{2} \gamma+129 \beta \gamma^{2}-51 \gamma^{3}\right)} \\
& +\left(791 \alpha(\beta-2 \gamma)\left(156 \beta^{4}-920 \beta^{3} \gamma+1755 \beta^{2} \gamma^{2}-1086 \beta \gamma^{3}+3 \gamma^{4}\right)\right. \\
& +\left(c_{k, 2}+c_{\{A, B\} \backslash k, 2}\right) \beta\left(-124388 \beta^{5}+1354780 \beta^{4} \gamma-5806421 \beta^{3} \gamma^{2}\right. \\
& \left.\left.+12237147 \beta^{2} \gamma^{3}-12678663 \beta \gamma^{4}+5164041 \gamma^{5}\right)\right] \\
& /\left(791\left(496 \beta^{6}-5040 \beta^{5} \gamma+19620 \beta^{4} \gamma^{2}-35388 \beta^{3} \gamma^{3}+25965 \beta^{2} \gamma^{4}+990 \beta \gamma^{5}-7119 \gamma^{6}\right)\right) \text {, } \\
& p_{k, 2}^{*}=\left[\frac{-54579 c_{k, 1}+44166 c_{k, 2}-121814 c_{\{A, B\} \backslash k, 1}-90304 c_{\{A, B\} \backslash k, 2}}{201705}\right. \\
& -\frac{\beta}{24 \beta-36 \gamma}\left(c_{k, 1}-c_{k, 2}+c_{\{A, B\} \backslash k, 1}-c_{\{A, B\} \backslash k, 2}\right)+\frac{10 \alpha+\beta \sum_{\substack{n \in\{A, B\} \\
j \in\{1,2\}}} c_{n j}}{60(2 \beta-5 \gamma)} \\
& +\frac{17 \alpha(3 \beta-4 \gamma)(\beta-2 \gamma)+\left(c_{k, 1}+c_{\{A, B\} \backslash k, 1}\right) \beta\left(77 \beta^{2}-349 \beta \gamma+386 \gamma^{2}\right)}{17\left(16 \beta^{3}-84 \beta^{2} \gamma+129 \beta \gamma^{2}-51 \gamma^{3}\right)} \\
& +\left(( c _ { k , 2 } + c _ { \{ A , B \} \backslash k , 2 } ) \beta \left(257224 \beta^{5}-2734368 \beta^{4} \gamma+11469404 \beta^{3} \gamma^{2}-23719599 \beta^{2} \gamma^{3}\right.\right. \\
& \left.+24175518 \beta \gamma^{4}-9709167 \gamma^{5}\right) \\
& \left.\left.+791 \alpha\left(152 \beta^{5}-936 \beta^{4} \gamma+1568 \beta^{3} \gamma^{2}+645 \beta^{2} \gamma^{3}-3648 \beta \gamma^{4}+2283 \gamma^{5}\right)\right)\right] \\
& /\left(1582\left(496 \beta^{6}-5040 \beta^{5} \gamma+19620 \beta^{4} \gamma^{2}-35388 \beta^{3} \gamma^{3}+25965 \beta^{2} \gamma^{4}+990 \beta \gamma^{5}-7119 \gamma^{6}\right)\right) \text {, } \\
& s_{11}^{*}=\frac{136843 c_{A 1}+81923 c_{A 2}+136843 c_{B 1}+81923 c_{B 2}}{403410} \\
& +\frac{\left(-c_{A 1}+c_{A 2}-c_{B 1}+c_{B 2}\right) \beta}{48 \beta-72 \gamma}+\frac{10 \alpha+\beta \sum_{\substack{n \in\{A, B\} \\
j \in\{1,2\}}} c_{n j}}{40(2 \beta-5 \gamma)} \\
& +\frac{\left(c_{A 1}+c_{B 1}\right) \beta\left(-88 \beta^{2}+445 \beta \gamma-531 \gamma^{2}\right)+17 \alpha\left(8 \beta^{2}-17 \beta \gamma+3 \gamma^{2}\right)}{68\left(16 \beta^{3}-84 \beta^{2} \gamma+129 \beta \gamma^{2}-51 \gamma^{3}\right)}
\end{aligned}
$$




$$
\begin{aligned}
& +\left(791 \alpha\left(528 \beta^{5}-4024 \beta^{4} \gamma+11106 \beta^{3} \gamma^{2}-12753 \beta^{2} \gamma^{3}+4200 \beta \gamma^{4}+1251 \gamma^{5}\right)\right. \\
& +\left(c_{A 2}+c_{B 2}\right) \beta\left(-210816 \beta^{5}+2334248 \beta^{4} \gamma-10152258 \beta^{3} \gamma^{2}+21685365 \beta^{2} \gamma^{3}\right. \\
& \left.\left.-22757664 \beta \gamma^{4}+9389889 \gamma^{5}\right)\right) \\
& /\left(1582\left(496 \beta^{6}-5040 \beta^{5} \gamma+19620 \beta^{4} \gamma^{2}-35388 \beta^{3} \gamma^{3}+25965 \beta^{2} \gamma^{4}+990 \beta \gamma^{5}-7119 \gamma^{6}\right)\right), \\
& s_{22}^{*}=\frac{-10283 c_{A 1}-493 c_{A 2}-10283 c_{B 1}-493 c_{B 2}}{28815}+\frac{\left(c_{A 1}-c_{A 2}+c_{B 1}-c_{B 2}\right) \beta}{48 \beta-72 \gamma}+\frac{10 \alpha+\beta \sum_{\substack{n \in\{A, B\} \\
j \in\{1,2\}}} c_{n j}}{40(2 \beta-5 \gamma)} \\
& +\frac{17 \alpha\left(18 \beta^{2}-55 \beta \gamma+39 \gamma^{2}\right)+\left(c_{A 1}+c_{B 1}\right) \beta\left(318 \beta^{2}-1423 \beta \gamma+1563 \gamma^{2}\right)}{68\left(16 \beta^{3}-84 \beta^{2} \gamma+129 \beta \gamma^{2}-51 \gamma^{3}\right)} \\
& +\left(\left(c_{A 2}+c_{B 2}\right) \beta\left(31328 \beta^{5}-325040 \beta^{4} \gamma+1327452 \beta^{3} \gamma^{2}-2663943 \beta^{2} \gamma^{3}+2622558 \beta \gamma^{4}-1010799 \gamma^{5}\right)\right. \\
& \left.+113 \alpha\left(368 \beta^{5}-2576 \beta^{4} \gamma+6048 \beta^{3} \gamma^{2}-4317 \beta^{2} \gamma^{3}-2382 \beta \gamma^{4}+3087 \gamma^{5}\right)\right) \\
& \text { / }\left(226\left(496 \beta^{6}-5040 \beta^{5} \gamma+19620 \beta^{4} \gamma^{2}-35388 \beta^{3} \gamma^{3}+25965 \beta^{2} \gamma^{4}+990 \beta \gamma^{5}-7119 \gamma^{6}\right)\right) \text {, } \\
& w_{k, 1}^{*}=\left[8 \beta^{5}\left(35 \alpha+\left(-35 c_{k, 2}+62 c_{\{A, B\} \backslash k, 1}+27 c_{\{A, B\} \backslash k, 2}\right) \beta\right)\right. \\
& -168 \beta^{4}\left(13 \alpha+6\left(-3 c_{k, 2}+5 c_{\{A, B\} \backslash k, 1}+2 c_{\{A, B\} \backslash k, 2}\right) \beta\right) \gamma \\
& +36 \beta^{3}\left(174 \alpha+\left(-356 c_{k, 2}+545 c_{\{A, B\} \backslash k, 1}+189 c_{\{A, B\} \backslash k, 2}\right) \beta\right) \gamma^{2} \\
& -9 \beta^{2}\left(863 \alpha+\left(-2951 c_{k, 2}+3932 c_{\{A, B\} \backslash k, 1}+981 c_{\{A, B\} \backslash k, 2}\right) \beta\right) \gamma^{3} \\
& +9 \beta\left(372 \alpha+\left(-2961 c_{k, 2}+2885 c_{\{A, B\} \backslash k, 1}-76 c_{\{A, B\} \backslash k, 2}\right) \beta\right) \gamma^{4} \\
& +9\left(27 \alpha+11\left(99 c_{k, 2}+10 c_{\{A, B\} \backslash k, 1}+109 c_{\{A, B\} \backslash k, 2}\right) \beta\right) \gamma^{5} \\
& \left.+9\left(81 c_{k, 2}-791 c_{\{A, B\} \backslash k, 1}-710 c_{\{A, B\} \backslash k, 2}\right) \gamma^{6}\right] \\
& /\left(2\left(496 \beta^{6}-5040 \beta^{5} \gamma+19620 \beta^{4} \gamma^{2}-35388 \beta^{3} \gamma^{3}+25965 \beta^{2} \gamma^{4}+990 \beta \gamma^{5}-7119 \gamma^{6}\right)\right) \text {, } \\
& w_{k, 2}^{*}=\frac{1}{53788}\left[-7910 c_{k, 1}+21981 c_{k, 2}-21357 c_{\{A, B\} \backslash k, 1}-18360 c_{\{A, B\} \backslash k, 2}\right. \\
& +\frac{791\left(17 \alpha(5 \beta-9 \gamma)(2 \beta-3 \gamma)+\left(c_{k, 1}+c_{\{A, B\} \backslash k, 1}\right) \beta\left(262 \beta^{2}-1197 \beta \gamma+1341 \gamma^{2}\right)\right)}{16 \beta^{3}-84 \beta^{2} \gamma+129 \beta \gamma^{2}-51 \gamma^{3}} \\
& +204(791 \alpha(5 \beta-9 \gamma)(2 \beta-5 \gamma)(2 \beta-3 \gamma)(\beta-2 \gamma)(\beta+\gamma) \\
& \left.+\left(c_{k, 2}+c_{\{A, B\} \backslash k, 2}\right) \beta\left(262 \beta^{2}-1197 \beta \gamma+1341 \gamma^{2}\right)\left(110 \beta^{3}-637 \beta^{2} \gamma+1144 \beta \gamma^{2}-629 \gamma^{3}\right)\right) \\
& \text { / } \left.\left(496 \beta^{6}-5040 \beta^{5} \gamma+19620 \beta^{4} \gamma^{2}-35388 \beta^{3} \gamma^{3}+25965 \beta^{2} \gamma^{4}+990 \beta \gamma^{5}-7119 \gamma^{6}\right)\right] \text {. }
\end{aligned}
$$

\section{C.3. MSS-RS-MBRB}

$$
\begin{aligned}
p_{k, 1}^{*}= & \frac{1}{19740}\left[10998 c_{k, 1}-9387 c_{k, 2}+2773 c_{\{A, B\} \backslash k, 1}-20902 c_{\{A, B\} \backslash k, 2}+\frac{329\left(10 \alpha+\beta \sum_{\substack{n \in\{A, B\} \\
j \in\{1,2\}}} c_{n j}\right)}{2 \beta-5 \gamma}\right. \\
& +\frac{1645\left(c_{k, 1}-c_{k, 2}+c_{\{A, B\} \backslash k, 1}-c_{\{A, B\} \backslash k, 2}\right) \beta}{-2 \beta+3 \gamma}+\frac{705\left(7 \alpha \gamma+\left(c_{k, 1}+c_{\{A, B\} \backslash k, 1}\right) \beta(-6 \beta+17 \gamma)\right)}{2 \beta^{2}-8 \beta \gamma+7 \gamma^{2}}
\end{aligned}
$$




$$
\begin{aligned}
& \left.+\frac{210\left(47 \alpha(\beta-2 \gamma)\left(8 \beta^{2}-32 \beta \gamma+29 \gamma^{2}\right)+\left(c_{k, 2}+c_{\{A, B\} \backslash k, 2}\right) \beta\left(320 \beta^{3}-2184 \beta^{2} \gamma+4782 \beta \gamma^{2}-3301 \gamma^{3}\right)\right)}{\left(8 \beta^{4}-64 \beta^{3} \gamma+183 \beta^{2} \gamma^{2}-220 \beta \gamma^{3}+94 \gamma^{4}\right)}\right], \\
& p_{k, 2}^{*}=\frac{-1128 c_{k, 1}+5187 c_{k, 2}-2773 c_{\{A, B\} \backslash k, 1}+1897 c_{\{A, B\} \backslash k, 2}}{4935}+\frac{\left(c_{k, 1}-c_{k, 2}+c_{\{A, B\} \backslash k, 1}-c_{\{A, B\} \backslash k, 2}\right) \beta}{24 \beta-36 \gamma} \\
& +\frac{10 \alpha+\beta \sum_{\substack{n \in\{A, B\} \\
j \in\{1,2\}}} c_{n j}}{60(2 \beta-5 \gamma)}+\frac{\left(c_{k, 1}+c_{\{A, B\} \backslash k, 1}\right) \beta(5 \beta-13 \gamma)+7 \alpha(\beta-2 \gamma)}{14\left(2 \beta^{2}-8 \beta \gamma+7 \gamma^{2}\right)} \\
& +\left(-264\left(c_{k, 2}+c_{\{A, B\} \backslash k, 2}\right) \beta^{4}+6 \beta^{2}\left(47 \alpha+305\left(c_{k, 2}+c_{\{A, B\} \backslash k, 2}\right) \beta\right) \gamma\right. \\
& \left.-3 \beta\left(376 \alpha+1355\left(c_{k, 2}+c_{\{A, B\} \backslash k, 2}\right) \beta\right) \gamma^{2}+2\left(517 \alpha+1421\left(c_{k, 2}+c_{\{A, B\} \backslash k, 2}\right) \beta\right) \gamma^{3}\right) \\
& /\left(94\left(8 \beta^{4}-64 \beta^{3} \gamma+183 \beta^{2} \gamma^{2}-220 \beta \gamma^{3}+94 \gamma^{4}\right)\right) \text {, } \\
& s_{11}^{*}=\frac{1363 c_{A 1}-532 c_{A 2}+1363 c_{B 1}-532 c_{B 2}}{4935}+\frac{\left(-c_{A 1}+c_{A 2}-c_{B 1}+c_{B 2}\right) \beta}{48 \beta-72 \gamma} \\
& +\frac{10 \alpha+\beta \sum_{\substack{n \in\{A, B\} \\
j \in\{1,2\}}} c_{n j}}{40(2 \beta-5 \gamma)}+\frac{-\left(c_{A 1}+c_{B 1}\right) \beta(\beta-4 \gamma)+7 \alpha(\beta-\gamma)}{28\left(2 \beta^{2}-8 \beta \gamma+7 \gamma^{2}\right)} \\
& +\frac{\left(47 \alpha(2 \beta-3 \gamma)\left(4 \beta^{2}-15 \beta \gamma+12 \gamma^{2}\right)+\left(c_{A 2}+c_{B 2}\right) \beta(4 \beta-9 \gamma)\left(14 \beta^{2}-57 \beta \gamma+51 \gamma^{2}\right)\right)}{\left(94\left(8 \beta^{4}-64 \beta^{3} \gamma+183 \beta^{2} \gamma^{2}-220 \beta \gamma^{3}+94 \gamma^{4}\right)\right)}, \\
& s_{22}^{*}=\frac{-517 c_{A 1}+1393 c_{A 2}-517 c_{B 1}+1393 c_{B 2}}{4935}+\frac{\left(c_{A 1}-c_{A 2}+c_{B 1}-c_{B 2}\right) \beta}{48 \beta-72 \gamma} \\
& +\frac{10 \alpha+\beta \sum_{\substack{n \in\{A, B\} \\
j \in\{1,2\}}} c_{n j}}{40(2 \beta-5 \gamma)}+\frac{\left(c_{A 1}+c_{B 1}\right) \beta(4 \beta-9 \gamma)+7 \alpha(2 \beta-3 \gamma)}{28\left(2 \beta^{2}-8 \beta \gamma+7 \gamma^{2}\right)} \\
& +\frac{\left(94 \alpha(\beta-\gamma)\left(3 \beta^{2}-12 \beta \gamma+11 \gamma^{2}\right)-\left(c_{A 2}+c_{B 2}\right) \beta(\beta-4 \gamma)\left(18 \beta^{2}-72 \beta \gamma+65 \gamma^{2}\right)\right)}{\left(94\left(8 \beta^{4}-64 \beta^{3} \gamma+183 \beta^{2} \gamma^{2}-220 \beta \gamma^{3}+94 \gamma^{4}\right)\right)}, \\
& l w_{k, 1}^{*}=\left[8 \beta^{3}\left(\alpha+\left(c_{k, 1}-c_{\{A, B\} \backslash k, 2}\right) \beta\right)+8 \beta^{2}\left(-6 \alpha+\left(-8 c_{k, 1}+c_{k, 2}+9 c_{\{A, B\} \backslash k, 2}\right) \beta\right) \gamma\right. \\
& +3 \beta\left(31 \alpha+\left(61 c_{k, 1}-18 c_{k, 2}-79 c_{\{A, B\} \backslash k, 2}\right) \beta\right) \gamma^{2} \\
& \left.+\left(-58 \alpha+\left(-220 c_{k, 1}+117 c_{k, 2}+337 c_{\{A, B\} \backslash k, 2}\right) \beta\right) \gamma^{3}+2\left(47 c_{k, 1}-40 c_{k, 2}-87 c_{\{A, B\} \backslash k, 2}\right) \gamma^{4}\right] \\
& /\left(2\left(8 \beta^{4}-64 \beta^{3} \gamma+183 \beta^{2} \gamma^{2}-220 \beta \gamma^{3}+94 \gamma^{4}\right)\right) \text {, } \\
& w_{k, 2}^{*}=\frac{1}{1316}\left[-235 c_{k, 1}+1302 c_{k, 2}-564 c_{\{A, B\} \backslash k, 1}+315 c_{\{A, B\} \backslash k, 2}\right. \\
& +\frac{94\left(\left(c_{k, 1}+c_{\{A, B\} \backslash k, 1}\right) \beta(5 \beta-13 \gamma)+7 \alpha(\beta-2 \gamma)\right)}{2 \beta^{2}-8 \beta \gamma+7 \gamma^{2}} \\
& +\left(7 \left(-360\left(c_{k, 2}+c_{\{A, B\} \backslash k, 2}\right) \beta^{4}+8 \beta^{2}\left(47 \alpha+313\left(c_{k, 2}+c_{\{A, B\} \backslash k, 2}\right) \beta\right) \gamma\right.\right.
\end{aligned}
$$




$$
\begin{aligned}
& \left.\left.-\beta\left(1504 \alpha+5603\left(c_{k, 2}+c_{\{A, B\} \backslash k, 2}\right) \beta\right) \gamma^{2}+6\left(235 \alpha+663\left(c_{k, 2}+c_{\{A, B\} \backslash k, 2}\right) \beta\right) \gamma^{3}\right)\right) \\
& \left./\left(8 \beta^{4}-64 \beta^{3} \gamma+183 \beta^{2} \gamma^{2}-220 \beta \gamma^{3}+94 \gamma^{4}\right)\right], \\
v_{11}^{*}= & {\left[8 \beta^{3}\left(\alpha+\left(c_{A 1}+c_{B 1}\right) \beta\right)+2 \beta^{2}\left(-21 \alpha+\left(-32 c_{A 1}+c_{A 2}-32 c_{B 1}+c_{B 2}\right) \beta\right) \gamma\right.} \\
& +3 \beta\left(23 \alpha+\left(61 c_{A 1}-4 c_{A 2}+61 c_{B 1}-4 c_{B 2}\right) \beta\right) \gamma^{2} \\
& +\left(-36 \alpha+\left(-220 c_{A 1}+23 c_{A 2}-220 c_{B 1}+23 c_{B 2}\right) \beta\right) \gamma^{3} \\
& \left.+2\left(47 c_{A 1}-7 c_{A 2}+47 c_{B 1}-7 c_{B 2}\right) \gamma^{4}\right] /\left(2\left(8 \beta^{4}-64 \beta^{3} \gamma+183 \beta^{2} \gamma^{2}-220 \beta \gamma^{3}+94 \gamma^{4}\right)\right), \\
v_{22}^{*}= & \frac{1}{1316}\left[-94 c_{A 1}+672 c_{A 2}-94 c_{B 1}+672 c_{B 2}+\frac{47\left(\left(c_{A 1}+c_{B 1}\right) \beta(4 \beta-9 \gamma)+7 \alpha(2 \beta-3 \gamma)\right)}{2 \beta^{2}-8 \beta \gamma+7 \gamma^{2}}\right. \\
& +\frac{\left(-7\left(c_{A 2}+c_{B 2}\right) \beta(4 \beta-9 \gamma)(4 \beta-7 \gamma)(\beta-4 \gamma)+658 \alpha(2 \beta-5 \gamma)(2 \beta-3 \gamma)(\beta-\gamma)\right)}{\left(8 \beta^{4}-64 \beta^{3} \gamma+183 \beta^{2} \gamma^{2}-220 \beta \gamma^{3}+94 \gamma^{4}\right)} .
\end{aligned}
$$

\section{REFERENCES}

[1] W.J. Adams and J.L. Yellen, Commodity bundling and the burden of monopoly. Q. J. Econ. 90 (1976) $475-498$.

[2] K.E. Atkinson, Numerical solutions of systems of linear equations, 2nd edition. In: An Introduction to Numerical Analysis. John Wiley \& Sons, New York, NY (1988), 508-515.

[3] R. Amir and A. Stepanova, Second-mover advantage and price leadership in Bertrand duopoly. Games Econ. Behav. 55 (2006) $1-20$.

[4] G. Aust and U. Buscher, Game theoretic analysis of pricing and vertical cooperative advertising of a retailer-duopoly with a common manufacturer. Cent. Eur. J. Oper. Res. 24 (2016) 127-147.

[5] A. Chakravarty, A. Mild and A. Taudes, Bundling decisions in supply chains. Eur. J. Oper. Res. 231 (2013) 617-630.

[6] X. Chen and X. Wang, Free or bundled: channel selection decisions under different power structures. Omega 53 (2015) 11-20.

[7] S.C. Choi, Price competition in a channel structure with a common retailer. Marketing Sci. 10 (1991) $271-296$.

[8] N. Economides, Mixed bundling in duopoly. Discussion Paper No. EC-93-29. Stern School of Business, New York University, New York, NY (1993).

[9] N. Economides and S.C. Salop, Competition and integration among complements, and network market structure. J. Ind. Econ. 40 (1991).

[10] R.N. Giri, S.K. Mondal and M. Maiti, Bundle pricing strategies for two complementary products with different channel powers. Ann. Oper. Res. 287 (2017) 701-725.

[11] S. Guidon, M. Wicki, T. Bernauer and K. Axhausen, Transportation service bundling' for whose benefit? Consumer valuation of pure bundling in the passenger transportation market. Transp. Res. Part A: Policy Pract. 131 (2020) 91-106.

[12] R. Gullu, G.-J. van Houtum, F. Zeynep Sargut and N. Erkip, Analysis of a decentralized supply chain under partial cooperation. Manuf. Serv. Oper. Manage. 7 (2005) 229-247.

[13] H. Jafari, S. Reza Hejazi and M. Rasti, Pricing decisions in dual-channel supply chain with one manufacturer and multiple retailers: A game-theoretic approach. RAIRO: OR 51 (2017) 1269-1287.

[14] T. Kawamori and M.H. Lin, The way of offering vertically differentiated airline services. Oper. Res. Lett. 40 (2012) 374-377.

[15] S. Keyhanian, A. Ahmadi and B. Karimi, A study on pricing under a coopetitive and duopolistic market of vertically differentiated products. Ind. Eng. Manage. Syst. 18 (2019) 776-793.

[16] S. Kjeldsen-Kragh, Demand conditions for differentiated products. In: International Economics: Trade and Investment. Copenhagen Business School Press, Copenhagen (2002) 202-205.

[17] H. Lee and S. Whang, Decentralized multi-echelon supply chains: Incentives and information. Manage. Sci. 45 (1999) 633-640.

[18] J.-C. Li, J.-H. Lu, Q.-L. Wang and C. Li, Quality and pricing decisions in a two-echelon supply chain with nash bargaining fairness concerns. Discrete Dyn. Nat. Soc. 2018 (2018) 4267305.

[19] X. Lin and Y.W. Zhou, Pricing policy selection for a platform providing vertically differentiated services with self-scheduling capacity. J. Oper. Res. Soc. 70 (2019) 1203-1218.

[20] L. Liu, M. Parlar and S.X. Zhu, Pricing and lead time decisions in decentralized supply chains. Manage. Sci. 53 (2007) 713-725.

[21] P. Ma, C. Zhang, X. Hong and H. Xu, Pricing decisions for substitutable products with green manufacturing in a competitive supply chain. J. Cleaner Prod. 183 (2018) 618-640.

[22] C. Matutes and P. Regibeau, Compatibility and bundling of complementary goods in a duopoly. J. Ind. Econ. 40 (1992) 37-54.

[23] W.G. Morrison, Product bundling and shared information goods: A pricing exercise. J. Econ. Edu. 47 (2016) $49-63$. 
[24] L. Pan and S. Zhou, Optimal bundling and pricing decisions for complementary products in a two-layer supply chain. J. Syst. Sci. Syst. Eng. 26 (2017) 732-752.

[25] B. Pierce and H. Winter, Pure vs. mixed commodity bundling. Rev. Ind. Org. 11 (1996) 811-821.

[26] J. Shao, H. Krishnan and S. Thomas McCormick, Incentives for transshipment in a supply chain with decentralized retailers. Manuf. Serv. Oper. Manage. 13 (2011) 361-372.

[27] S.M. Shugan, J. Moon, Q. Shi and N.S. Kumar. Product Line Bundling: Why Airlines Bundle High-End While Hotels Bundle Low-End. Marketing Sci. 36 (2016) 124-139.

[28] J. Wei, J. Zhao and Y. Li, Pricing decisions for complementary products with firms' different market powers. Eur. J. Oper. Res. 224 (2013) 507-519.

[29] D. Wu, Joint pricing-servicing decision and channel strategies in the supply chain. Cent. Eur. J. Oper. Res. 19 (2011) 99-137.

[30] C.-H. Wu, C.-W. Chen and C.-C. Hsieh, Competitive pricing decisions in a two-echelon supply chain with horizontal and vertical competition. Int. J. Prod. Econ. 135 (2012) 265-274.

[31] M. Xue and J. Zhang, Impacts of heterogeneous environment awareness and power structure on green supply chain. $R A I R O: O R$ 52 (2018) 143-157.

[32] Y. Yan, R. Zhao and Y. Lan, Asymmetric retailers with different moving sequences: Group buying vs. individual purchasing. Eur. J. Oper. Res. 261 (2017) 903-917.

[33] F. Zhang, Competition, cooperation, and information sharing in a two-echelon assembly system. Manuf. Serv. Oper. Manage. 8 (2006) 273-291.

[34] J. Zhao, J. Wei and Y. Li, Pricing decisions for substitutable products in a two-echelon supply chain with firms' different channel powers. Int. J. Prod. Econ. 153 (2014) 243-252.

[35] S. Zhou, B. Song and S. Gavirneni, Bundling decisions in a two-product duopoly - Lead or follow? Eur. J. Oper. Res. 284 (2020) 980-989. 